\title{
Interaction corrections to the thermal transport coefficients in disordered metals: quantum kinetic equation approach.
}

\author{
G. Catelani and I.L. Aleiner \\ Physics Department, Columbia University, New York, NY 10027
}

(Dated: September 17, 2018)

\begin{abstract}
We consider the singular electron-electron interaction corrections to the transport coefficients in disordered metals to test the validity of the Wiedemann-Franz law. We develop a local, quantum kinetic equation approach in which the charge and energy conservation laws are explicitly obeyed. To obtain the local description, we introduce bosonic distribution functions for the neutral, lowenergy collective modes (electron-hole pairs). The resulting system of kinetic equations enables us to distinguish between the different physical processes involved in the charge and energy transport: elastic electron scattering affects both, while the inelastic processes influence only the latter. Moreover, the neutral bosons, though incapable of transporting charge, contribute significantly to the energy transport. In our approach we calculate on equal footing the electrical and thermal conductivities and the specific heat in any dimension. We found that the Wiedemann-Franz law is always violated by the interaction corrections; the violation is larger for one- and two-dimensional systems in the diffusive regime $T \tau \ll \hbar$ and it is due to the energy transported by the neutral bosons. For two-dimensional systems in the quasi-ballistic regime $T \tau \gg \hbar$ the inelastic scattering of the electron on the bosons also contributes to the violation.

PACS numbers: $71.10 . \mathrm{Ay}, 72.10 . \mathrm{Bg}, 72.15 . \mathrm{Eb}$
\end{abstract}

\section{INTRODUCTION}

It is well known that the measurement of the thermal transport coefficient may provide additional information about the scattering processes in disordered metals. In particular, the Wiedemann-Franz ${ }^{1}$ law holds as long as elastic scattering ${ }^{35}$ dominates in the system:

$$
\mathrm{L}=\frac{\kappa}{\sigma T}=\frac{\pi^{2}}{3 e^{2}}
$$

where $\kappa$ and $\sigma$ are respectively the thermal and electric conductivities in the system, $T$ is the temperature in energy units $\left(k_{B}=1\right)$ and $e$ is the electron charge. On the other hand for the deeply inelastic forward scattering the Wiedemann-Franz law is violated ${ }^{2}$, so that the Lorentz number $\mathrm{L}$ is smaller than the universal value, $\mathrm{L}<\frac{\pi^{2}}{3 e^{2}}$.

Historically, the transport (in particular thermal transport) coefficients were first being calculated using the Boltzmann equation $(\mathrm{BE})^{3}$. The advantage of this approach is that it allows for the clear separation of the scale in the problem: a particle moves freely most of the time and rarely scatters on other particles or impurities. The BE is applicable on the time scale much larger than the time it takes for the scattering to happen, so all the scattering events are encoded into the local collision integral. All the quantum mechanical part of the calculation is reduced then into the solution of the scattering problems for the relevant physical processes. This gives the precise form of the collision integral but it does not affect the general structure of the BE. The great advantage of the $\mathrm{BE}$ is that its structure illuminates the relevant conservation laws.

In the late 50 s an alternative approach became popular - the so called Kubo formulas ${ }^{4}$. In this approach the transport equation is not derived but rather the connec- tion of the transport coefficient to the equilibrium correlation function of certain current operators is used. (The Kubo approach to the thermal transport was claimed to be put on rigorous footing by Luttinger ${ }^{5}$ based on the assumption that there exists some spatial scale in the system so that for perturbations smooth on that scale the gradient expansion is possible). Being exact, the Kubo formulas are formally applicable even in the regime where the transport equation can not be justified (separation of the evolution into free motion and rare collisions is not possible).

However, in practice, the possibility of explicit calculation within the Kubo formula is somewhat limited. The most spectacular results of the Kubo-formula calculations - such as Maki-Thompson ${ }^{6,7}$, Aslamazov-Larkin ${ }^{8}$ and weak localization ${ }^{9}$ corrections to the electrical conductivity - require a small parameter which is the same parameter that determines the applicability of the Boltzmann equation. It means that all those effects can be also described in terms of quantum corrections to the collision integral (for weak localization it was done in Ref. 13). The most relevant for this paper effect - the Altshuler-Aronov ${ }^{10}$ interaction correction to the electrical conductivity in two dimensions ${ }^{11,14}$

$$
\delta \sigma_{A A}=-\frac{e^{2}}{2 \pi^{2} \hbar} \ln \left(\frac{\hbar}{T \tau}\right)\left[1+3\left(1-\frac{1}{F_{0}^{\sigma}} \ln \left(1+F_{0}^{\sigma}\right)\right)\right]
$$

originates from the elastic scattering of the electrons on the self-consistent potential (Friedel oscillation) ${ }^{15,16}$, and it can be once again obtained from the correction to the collision integral ${ }^{17}$.

The success of the Kubo formulas in the description of the quantum and interaction effects in thermal transport is by far more modest and controversial. Particularly, despite a 20-years history there is no consensus on the 
answer to a natural question: how does the logarithmic correction to the conductivity (1.2) translate into a correction to the Wiedemann-Franz law (1.1)?

A first attempt at answering this question was made by Castellani at al. ${ }^{19}$ by analyzing Ward identities for a disordered Fermi liquid; they found that the WiedemannFranz law should hold for interacting disordered electrons. Their claim was later disputed by Livanov et al. ${ }^{20}$ : in a "quantum kinetic equation" approach, ${ }^{36}$ a logarithmic divergence for the thermal conductivity in two dimensions was found to have even the sign contrary to the Wiedemann-Franz law. More recently Niven and Smith $^{22}$ applied Kubo formula and found again a logarithmically divergent contribution (for the Coulomb but not the short-range interaction) in addition to the one that follows from the Wiedemann-Franz law.

The reason for this confusion in the literature is twofold. Technically, the identification of the correct form of the current operator is complicated by the presence of the electron-electron interaction (the energy current operator in the form defined by Luttinger ${ }^{5}$ is cumbersome to use due to the presence of the additional disorder and interaction potentials in it, whereas the superficially more elegant expression in the Matsubara frequency representation in fact does not correspond to any conservation law for the interacting system and violates gauge invariance - see Appendix B). Physically, the use of the diagrammatic calculation within the Kubo formula prevents one from a clear identification of the relevant scattering processes, because each diagram taken separately describes some mixture of such processes and does not have its own physical meaning.

This situation calls for the development of the kinetic equation description, which takes into account the interaction correction of the Altshuler-Aronov type both for the electric and thermal transport. The advantage of this approach is that it makes possible to keep track of the conservation laws explicitly and thus excludes any ambiguity in the definition of the currents. This paper is devoted to the development and application of this machinery.

We will use units with $\hbar=1$ throughout the paper and we will restore the Planck constant in the final results only. The remainder of the paper is organized as follows: in Sec. II we discuss some general features of the kinetic equation approach using a simple "toy model". In Sec. III, we present our final expression for the kinetic equation describing interacting electrons in disordered metals. Section IV summarizes the results for the thermal conductivity and the specific heat obtained by solving the kinetic equation. The derivation of the kinetic equation is presented in Sec. V, while the calculation of the transport coefficients and the specific heat is given in Sec. VI. Some mathematical details are relegated into Appendices.

\section{STRUCTURE OF THE KINETIC EQUATION: CURRENTS AND SPECIFIC HEAT}

The purpose of this section is to show how the structure of the kinetic equation permits the proper identification of the relevant currents. We will remind how to calculate the specific heat from the kinetic equation once the conservation laws are obtained (this enables a direct check against the much simpler thermodynamic calculation). We will discuss the locality requirement for a proper kinetic equation. The latter requirement will dictate the number of necessary degrees of freedom (i.e. independent distribution functions) which should be introduced into the kinetic description.

\section{A. Kinetic equation and conservation laws}

As a concrete example, we consider here electron-like and hole-like excitations coupled to neutral bosons in the presence of an external electric field $\boldsymbol{E}$. (As we will see later, a system of interacting electron can be effectively described at low temperatures by such a coupled system for the scattering at small momentum transfer in a particle-hole channel.) The kinetic equations for electrons and bosons have the form:

$$
\begin{gathered}
{\left[\frac{\partial}{\partial t}+v_{F} \boldsymbol{n} \cdot \boldsymbol{\nabla}+e v_{F} \boldsymbol{n} \cdot \boldsymbol{E} \frac{\partial}{\partial \epsilon}\right] f=S t_{e}\{f, N\}} \\
{\left[\frac{\partial}{\partial t}+v(\omega) \boldsymbol{n} \cdot \boldsymbol{\nabla}\right] N=S t_{b}\{f, N\}}
\end{gathered}
$$

where $f=f(\epsilon, \boldsymbol{n} ; t, \boldsymbol{r})$ is the distribution function for the electrons with charge $e, v_{F}$ is the Fermi velocity, and $\boldsymbol{n}$ is the direction of the momentum. Energy $\epsilon$ is counted from the Fermi level so that $f(\epsilon>0)$ describes the electronlike excitations and $1-f(-\epsilon), \epsilon>0$ corresponds to the hole-like excitations. Having in mind only singular in $T$ corrections, we neglect the dependence of the electron velocity on energy (electron-hole asymmetry) as it does not introduce anything but small correction regular in powers of $T^{2}$.

The bosonic function $N=N(\omega, \boldsymbol{n} ; t, \boldsymbol{r})$ is the distribution function for the bosons with velocity $\boldsymbol{v}(\omega)$. All the interaction effects are included into the collision integrals $S t_{e}$ and $S t_{b}$; for example, an electron-like excitation can decay into a less energetic electron and a neutral boson, or an electron and a hole can annihilate into a neutral bosons, etc. By locality, the collision integrals depend on the same variables as the distribution functions, i.e. $S t_{e}=S t_{e}(\epsilon, \boldsymbol{n} ; t, \boldsymbol{r})$ and $S t_{b}=S t_{b}(\omega, \boldsymbol{n} ; t, \boldsymbol{r})$.

In thermodynamic equilibrium and $\boldsymbol{E}=0$ the Fermi function for fermions and the Planck function for the neutral bosons

$$
f_{F}(\epsilon)=\frac{1}{\exp (\epsilon / T)+1} ; \quad N_{P}(\omega)=\frac{1}{\exp (\omega / T)-1} ;
$$


solve the kinetic equation. The temperature $T$ here is a constant determined by the initial conditions for the kinetic equation.

Being the effective description for the slow dynamics of the original quantum mechanical system, the kinetic equation must respect the conservation laws of the original system: (i) total charge conservation and (ii) total energy conservation. Those two conditions are enforced by the requirements for the collision integrals

$$
\int d \epsilon \nu\left\langle S t_{e}\{f, N\}\right\rangle_{\boldsymbol{n}}=0
$$

and

$$
\int d \epsilon \epsilon \nu\left\langle S t_{e}\{f, N\}\right\rangle_{\boldsymbol{n}}+\int d \omega \omega b(\omega)\left\langle S t_{b}\{f, N\}\right\rangle_{\boldsymbol{n}}=0 .
$$

Here $\nu$ is the density of states (DoS) of the electrons (we will neglect its energy dependence)and $b(\omega)$ is the density of states of the bosons. We also introduced the short-hand notation for the angular integral

$$
\langle\ldots\rangle_{n} \equiv \int \frac{d \boldsymbol{n}}{\Omega_{d}} \ldots,
$$

where $\Omega_{d}$ is the total solid angle in $d$ dimensions.

Let the electron density $\rho$ be given by:

$$
\rho(t, \boldsymbol{r})=e \nu \int d \epsilon\langle f(\epsilon, \boldsymbol{n} ; t, \boldsymbol{r})\rangle_{\boldsymbol{n}} .
$$

Integrating Eq. (2.1a) over the energy and the direction of the momentum and using Eq. (2.3a) we arrive at the continuity equation

$$
\frac{\partial \rho}{\partial t}+\nabla \cdot \boldsymbol{j}=0
$$

with the electron current density defined as

$$
\boldsymbol{j}(t, \boldsymbol{r})=e \nu v_{F} \int d \epsilon\langle\boldsymbol{n} f(\epsilon, \boldsymbol{n} ; t, \boldsymbol{r})\rangle_{\boldsymbol{n}} .
$$

(Strictly speaking Eq. (2.6) fixes only the longitudinal component of the current - an arbitrary curl may be added to Eq. (2.7). We will not consider the effect of the magnetic field here, so we will disregard such magnetization currents.)

We turn now to the analysis of the energy conservation. We multiply Eq. (2.1a) by $\nu \epsilon$ and integrate over $\epsilon$ and $\boldsymbol{n}$. Next, we multiply Eq. (2.1b) by $\omega b(\omega)$ and integrate over the $\boldsymbol{\omega}$ and $\boldsymbol{n}$. Adding the two results together we obtain, with the help of Eqs. (2.3b) and (2.7)

$$
\frac{\partial u_{t o t}}{\partial t}+\nabla \cdot \boldsymbol{j}_{t o t}^{\epsilon}=\boldsymbol{j} \cdot \boldsymbol{E}
$$

where

$$
\begin{aligned}
& u_{t o t}=u_{e}(t, \boldsymbol{r})+u_{b}(t, \boldsymbol{r}) \\
& u_{e}(t, \boldsymbol{r})=\nu \int d \epsilon \epsilon\langle f(\epsilon, \boldsymbol{n} ; t, \boldsymbol{r})\rangle_{\boldsymbol{n}} \\
& u_{b}(t, \boldsymbol{r})=\int d \omega \omega b(\omega)\langle N(\omega, \boldsymbol{n} ; t, \boldsymbol{r})\rangle_{\boldsymbol{n}}
\end{aligned}
$$

and

$$
\begin{aligned}
& \boldsymbol{j}_{t o t}^{\epsilon}=\boldsymbol{j}_{e}^{\epsilon}+\boldsymbol{j}_{b}^{\epsilon} \\
& \boldsymbol{j}_{e}^{\epsilon}(t, \boldsymbol{r})=v_{F} \nu \int d \epsilon \epsilon\langle\boldsymbol{n} f(\epsilon, \boldsymbol{n} ; t, \boldsymbol{r})\rangle_{\boldsymbol{n}} \\
& \boldsymbol{j}_{b}^{\epsilon}(t, \boldsymbol{r})=\int d \omega \omega b(\omega) v(\omega)\langle\boldsymbol{n} N(\omega, \boldsymbol{n} ; t, \boldsymbol{r})\rangle_{\boldsymbol{n}}
\end{aligned}
$$

The right hand side of Eq. (2.8) is nothing but the Joule heat. For the homogeneous system the gradient term in the left hand side vanishes, and by virtue of the energy conservation the expression (2.9) must be identified with the total energy density of the system. On the other hand, for $\boldsymbol{E}=0 \mathrm{Eq}$. (2.8) has the form of the continuity equation for the energy density; therefore Eqs. (2.10) has to be identified with the total energy current density. This statement is not entirely trivial. One could imagine that for an interacting system the DoS entering into the expressions for the charge (2.5) and for the energy density (2.9) are renormalized differently. The energy conservation equation (2.8) excludes such a possibility.

The conservation of energy (2.8) is valid for any rate of the energy flow in and out of the system. On the other hand, the collision integrals in Eqs. (2.1) define a certain time scale $\tau_{i n}$ - the dynamics slow on the scale of $\tau_{i n}$ can be characterized by the distribution functions $(2.2)$ with the temperature dependent on time, $T(t)$ [corrections to such an adiabatic description are of the order of $\left.\tau_{i n} \partial_{t} \ln T\right]$. Substituting such form of the distribution function in Eqs. (2.9), and the result in Eq. (2.8), we find for the homogeneous system

$$
C_{V}(T) \frac{\partial T}{\partial t}=\boldsymbol{j} \cdot \boldsymbol{E}
$$

where

$$
C_{V}=\frac{\partial}{\partial T}\left[\nu \int d \epsilon \epsilon f_{F}(\epsilon)+\int d \omega \omega b(\omega) N_{P}(\omega)\right]
$$

is nothing but the specific heat of the system. The latter quantity may be calculated independently by applying the standard diagrammatic technique for the equilibrium systems. The agreement of such a calculation with the structure of the kinetic equation result (2.12) will be the most important check of the consistency of our description of the thermal transport.

\section{B. Locality of the kinetic equation and the number of degrees of freedom}

The local in space and time form of the collision integrals is clearly a simplified description. Actually the collision integral may be nonlocal on the time scale of the order of $\hbar / T$ and on the spatial scale of the order of $\hbar v_{F} / T$. We will call such a description local, and the 
description where the non-locality is involved on larger spatial and time scales - non-local.

The number of distribution functions to be introduced into the description is governed by the locality of the kinetic equation. Let us use the model of Eqs. (2.1) to illustrate the point. We had a local description in terms of the fermionic and bosonic distribution functions. One can, however, try to eliminate the bosonic distribution function and obtain a description in terms of the electronic degrees of freedom only.

Assuming that the deviation of the distribution function from its equilibrium value is small, one can linearize the bosonic collision integral to the form

$$
S t_{b}\{f, N\}=-\hat{I}(N-\tilde{N}\{f\})
$$

where $\hat{I}$ is some positive definite integral operator and $\tilde{N}\{f\}$ is the functional of the fermionic distribution function $f(\epsilon)$, such that for $f(\epsilon)=f_{F}(\epsilon), \tilde{N}(\omega)=N_{P}(\omega)$. Using Eq. (2.13) one can formally solve Eq. (2.1b)

$$
N=\frac{1}{\frac{\partial}{\partial t}+v(\omega) \boldsymbol{n} \cdot \boldsymbol{\nabla}+\hat{I}} \hat{I} \tilde{N}\{f\}
$$

Substituting Eq. (2.14) into Eq. (2.1a) we apparently obtain the kinetic equation in terms of the electron distribution function only

$$
\begin{gathered}
{\left[\frac{\partial}{\partial t}+v_{F} \boldsymbol{n} \cdot \boldsymbol{\nabla}+e v_{F} \boldsymbol{n} \cdot \boldsymbol{E} \frac{\partial}{\partial \epsilon}\right] f=S t_{e}^{?}\{f\}} \\
S t_{e}^{?}\{f\} \equiv S t_{e}\left\{f, \frac{1}{\frac{\partial}{\partial t}+v(\omega) \boldsymbol{n} \cdot \boldsymbol{\nabla}+\hat{I}} \hat{I} \tilde{N}\{f\}\right\}
\end{gathered}
$$

If we are interested in the linear response to a weak and smooth external perturbation, the description in terms of this single kinetic equation is completely equivalent to the original coupled system (2.1). However, there are clearly drawbacks: the presence of the integral operator $\hat{I}$ in the collision integral makes it non-local on the scale determined by the kinetic equation itself rather than by the temperature. Moreover, though it is still easy to identify the continuity equation for the electron charge using Eq. (2.3a), there is no longer a relation similar to Eq. (2.3b). This is why the analysis of the energy conservation law becomes cumbersome: the calculation of specific heat and energy current requires the time expansion of the collision integral, which in turns seems to require the knowledge of the concrete form of the inelastic collision integral.

The example we have just considered is somewhat trivial because the separation of the system into the fermionic and bosonic modes was given from the very beginning. The problem we are dealing with in this paper is how to include the collective modes of the interacting electron system into the kinetic equation. Indeed, in this case any calculation will give the result in terms of the electronic distribution function only, and it is not clear a priori how to introduce the occupation numbers for the collective modes into the description.

As we will show, it may be possible to reverse our previous argument. We will consider a system of interacting electrons and we will find that the interactions are described by a non-local collision integral. Therefore we will introduce bosonic degrees of freedom that will enable us to rewrite the non-local kinetic equation in terms of coupled, local kinetic equations. Then we will be able to identify the energy density and energy current density as sums of fermionic and bosonic contributions. The concrete example will be discussed briefly in the following subsection.

\section{Degrees of freedom for the kinetics of disordered Fermi liquid}

We now focus on the disordered, interacting Fermi liquid. For simplicity, we consider the interaction in the singlet channel only. Our goal is to show that the thermodynamic result for the interaction correction to the specific heat has indeed the kinetic equation structure (2.12). As a result, we will be able to determine the necessary number of bosonic degrees of freedom for the local kinetic equation. For the paper to be self-contained, we briefly review the thermodynamic approach, referring the reader to the literature ${ }^{33}$ for further details.

The thermodynamic calculation of the specific heat $C_{V}$ is based on the relation between $C_{V}$ and the thermodynamic potential $\Omega$ :

$$
C_{V}=-T \frac{\partial^{2} \Omega}{\partial T^{2}}
$$

The thermodynamic potential can be written as the sum of the thermodynamic potential for non-interacting quasiparticle $\Omega_{0}$ and a correction $\delta \Omega$ associated with the soft modes in the system. Keeping such a correction is legitimate because it turns out to be a more singular function of temperature than the $T^{3}$ correction due to the electron-hole asymmetry.

The correction $\delta \Omega$ is given by the sum of the so-called ring diagrams, see Fig. 1. The Matsubara representation for this diagram is

$$
\delta \Omega=\frac{T}{2} \sum_{\omega_{n}} \int \frac{d^{d} q}{(2 \pi)^{d}} \ln \left(1+\frac{F}{\nu} \Pi\left(i\left|\omega_{n}\right|, \boldsymbol{q}\right)\right)
$$

Here $F$ is the interaction constant, $\omega_{n}=2 \pi T n$ are the bosonic Matsubara frequencies and $\Pi$ is the polarization operator. The explicit expression for this operator is not important for the present discussion and will be given later, see Eq. (5.43a).

A straightforward calculation, relegated to Appendix A, enables us to rewrite Eq. (2.17) as

$$
\delta \Omega=-\int \frac{d \omega}{2 \pi}\left(\frac{1}{2} \operatorname{coth} \frac{\omega}{2 T}\right) \int \frac{d^{d} q}{(2 \pi)^{d}} \operatorname{Im} \operatorname{Tr}\left[\ln \hat{\mathcal{L}}^{\rho}-\ln \hat{\mathcal{L}}^{g}\right],
$$



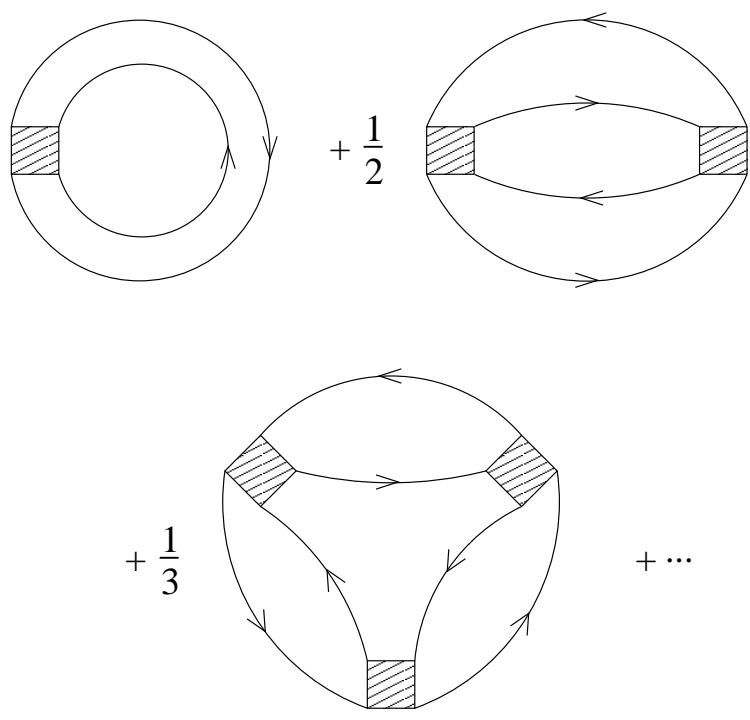

FIG. 1: Leading singular contribution to the thermodynamic potential for the clean system. The shaded box corresponds to $F / \nu$, defined through the two particle vertex $\Gamma^{\omega}$, see Ref. 26; the solid lines are coherent parts of the electron Green's functions. For the disordered system, the polarization bubbles should be dressed by the impurity scattering ${ }^{12}$.

The explicit expressions for the bosonic propagators $\mathcal{L}^{\rho}$, $\mathcal{L}^{g}$ are not relevant [they can be found in Eq. (3.11) the trace should be understood as a sum or integration over all variables other than $\omega, \boldsymbol{q}$ ]; we just mention here that $\mathcal{L}^{\rho}=\mathcal{L}^{g}$ in the absence of interaction, $F=0$. Substituting Eq. (2.18) into Eq. (2.16) and performing an integration over $\omega$ by parts, we find

$$
\delta C_{V}=\frac{\partial}{\partial T} \int_{0}^{\infty} d \omega \omega N_{P}(\omega)\left[b^{\rho}(\omega)-b^{g}(\omega)\right]
$$

where the densities of states are defined as

$$
\begin{aligned}
& b^{\rho}(\omega)=\frac{1}{\pi} \operatorname{Im} \int \frac{d^{d} q}{(2 \pi)^{d}} \partial_{\omega} \operatorname{Tr} \ln \mathcal{L}^{\rho} \\
& b^{g}(\omega)=\frac{1}{\pi} \operatorname{Im} \int \frac{d^{d} q}{(2 \pi)^{d}} \partial_{\omega} \operatorname{Tr} \ln \mathcal{L}^{g} .
\end{aligned}
$$

The function $b^{\rho}(\omega)$ has the physical meaning of the density of states (DoS) of the bosonic degrees of freedom in the system (soft electron-hole pairs). The function $b^{g}(\omega)$ has the meaning of the density of states of fictitious bosons (we will call them "ghosts") which describe the soft electron-hole pairs in the absence of interaction. The physical meaning of the minus sign in front of $b^{g}(\omega)$ is that with the formation of the collective modes some degrees of freedom are removed from the description of the non-interacting system; the introduction of the ghost bosons in the last term in Eq. (2.19a) takes this reduction into account.

Comparison of Eq. (2.19a) with Eqs. (2.12) and (2.9) hints the following expression for the contribution of the collective modes to the energy density in the nonequilibrium case

$$
u_{b}=\int_{0}^{\infty} d \omega \omega\left[N^{\rho}(\omega) b^{\rho}(\omega)-N^{g}(\omega) b^{g}(\omega)\right],
$$

where $N^{\rho}=N^{g}=N_{P}$ in the equilibrium and they have to be found from some kinetic equation otherwise [this definition requires that Eq. (2.8) holds for an arbitrary distribution function]. A similar expression can be obtained for the contribution due to the interaction in the triplet channel by introducing the additional propagator $\mathcal{L}^{\sigma}$ and distribution function $N^{\sigma}$. This means that the proper local kinetic equation must include four distribution functions: one for fermions, $f(\epsilon)$, and three for bosons, $N^{\rho, \sigma, g}(\omega)$. In the subsequent sections we will be able to derive such a description.

\section{FINAL FORM OF THE KINETIC EQUATION AND SCATTERING PROCESSES}

This section summarizes the final form of the quantum kinetic equation, the conservation laws and the corresponding currents. The derivation of these results is presented in Sec. V.

In accord with the previous section, the kinetics of the system is described by the electronic distribution function $f(\epsilon, \boldsymbol{n} ; t, \boldsymbol{r})$, the "distribution functions" of the bosonic singlet and triplet excitations, $\hat{N}^{\rho}$ and $\hat{N}^{\sigma}$, and the "distribution function" of the ghost excitation, $\hat{N}^{g}$.

The electron distribution function $f(\epsilon, \boldsymbol{n} ; t, \boldsymbol{r})$ is diagonal in the space of the momentum directions. On the contrary, the bosonic excitations are characterized by the density matrices $N^{\alpha}\left(\omega, \boldsymbol{q} ; \boldsymbol{n}_{i}, \boldsymbol{n}_{j} ; t, \boldsymbol{r}\right)[\alpha=\rho, \sigma, g]$ which may be not diagonal in the space of the momentum direction $\boldsymbol{n}$. Only in the thermal equilibrium

$$
\begin{aligned}
& f_{e q}(\epsilon, \boldsymbol{n} ; t, \boldsymbol{r})=f_{F}(\epsilon), \\
& N_{e q}^{\alpha}\left(\omega, \boldsymbol{q} ; \boldsymbol{n}_{i}, \boldsymbol{n}_{j} ; t, \boldsymbol{r}\right)=\Omega_{d} \delta\left(\widehat{\boldsymbol{n}_{i} \boldsymbol{n}_{j}}\right) N_{P}(\omega),
\end{aligned}
$$

with the Fermi and Plank distribution functions given by Eq. (2.2), the matrices $N^{\alpha}\left(\omega, \boldsymbol{q} ; \boldsymbol{n}_{i}, \boldsymbol{n}_{j} ; t, \boldsymbol{r}\right)$ acquire the diagonal form ${ }^{37}$. However even out of equilibrium these matrices have the property

$$
\begin{aligned}
& N^{\alpha}\left(\omega, \boldsymbol{q} ; \boldsymbol{n}_{i}, \boldsymbol{n}_{j}\right) \\
& \quad=-\left[N^{\alpha}\left(-\omega,-\boldsymbol{q} ; \boldsymbol{n}_{j}, \boldsymbol{n}_{i}\right)+\Omega_{d} \delta\left(\widehat{\boldsymbol{n}_{i} \boldsymbol{n}_{j}}\right)\right] .
\end{aligned}
$$

(hereafter, the spectator $t, \boldsymbol{r}$ variables might be suppressed.)

Strictly speaking, $f(\epsilon, \boldsymbol{n} ; t, \boldsymbol{r})$ is the $2 \times 2$ density matrix in the spin space and $N^{\sigma}$ is the $3 \times 3$ density matrix in the angular momentum $L=1$ space; however this will not be important in further calculations and we will write the equations for the diagonal components only. To account for the three folded degeneracy of the triplet mode we will explicitly introduce factors of 3 in the corresponding collision integrals and currents. 
For the sake of compactness, we will use an operator notation for matrices in the space of momentum direction, so that for example $\hat{N}$ should be understood as an operator acting on a function $a\left(\boldsymbol{n}_{i}\right)$ as follows:

$$
[\hat{N} a]\left(\boldsymbol{n}_{i}\right) \equiv \int \frac{d \boldsymbol{n}_{j}}{\Omega_{d}} N\left(\boldsymbol{n}_{i}, \boldsymbol{n}_{j}\right) a\left(\boldsymbol{n}_{j}\right) .
$$

The kinetic equation for the electrons in the electric field $\boldsymbol{E}$ (we will not consider the magnetic field effects) has the canonical form:

$$
\begin{aligned}
{\left[\partial_{t}+\boldsymbol{v}\right.} & \left.\cdot \nabla+e \boldsymbol{v} \cdot \boldsymbol{E} \frac{\partial}{\partial \epsilon}\right] f(\epsilon, \boldsymbol{n} ; t, \boldsymbol{r})=\widehat{\mathrm{St}}_{e}(\epsilon, \boldsymbol{n} ; t, \boldsymbol{r}) ; \\
\widehat{\mathrm{St}}_{e} & =\widehat{\mathrm{St}}_{\tau} f+\widehat{\mathrm{St}}^{e-\rho}\left\{f, N^{\rho}\right\}+3 \widehat{\mathrm{St}}^{e-\sigma}\left\{f, N^{\sigma}\right\} \\
& -4 \widehat{\mathrm{St}}^{e-g}\left\{f, N^{g}\right\}+\widehat{\mathrm{St}}^{e-e}\{f\},
\end{aligned}
$$

where the first term on the right hand side is the "bare" collision integral

$$
\mathrm{St}_{\tau}\left(\boldsymbol{n}_{i}, \boldsymbol{n}_{j}\right)=\frac{1}{\tau\left(\theta_{i j}\right)}-\delta\left(\widehat{\boldsymbol{n}_{i} \boldsymbol{n}_{j}}\right) \int \frac{d \boldsymbol{n}_{k}}{\tau\left(\theta_{i k}\right)},
$$

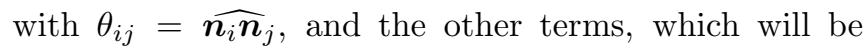
written shortly, take into account the interaction effects. by:

The bosonic distributions, for $\alpha=\rho, \sigma, g$, are governed

$$
\begin{aligned}
& \omega\left[\left\{\frac{1}{1+\hat{F}^{\alpha}} ; \partial_{t} \hat{N}^{\alpha}\right\}+\left\{\hat{\boldsymbol{s}}^{\alpha}(\omega, \boldsymbol{q}) ; \boldsymbol{\nabla} \hat{N}^{\alpha}\right\}\right. \\
& \left.+i\left[\hat{H}_{\mathrm{e}-\mathrm{h}}^{\alpha}(\omega, \boldsymbol{q}) ; \hat{N}^{\alpha}\right]\right]=\widehat{\mathrm{St}}^{\alpha-e}\left\{N^{\alpha}, f\right\}\left(\omega, \boldsymbol{q} ; \boldsymbol{n}_{i}, \boldsymbol{n}_{j} ; t, \boldsymbol{r}\right)
\end{aligned}
$$

where the commutator and anticommutator are defined as

$$
\{\hat{A} ; \hat{B}\} \equiv \frac{1}{2}(\hat{A} \hat{B}+\hat{B} \hat{A}) ; \quad[\hat{A} ; \hat{B}] \equiv \hat{A} \hat{B}-\hat{B} \hat{A} .
$$

The operators $\hat{H}_{\mathrm{e}-\mathrm{h}}^{\alpha}$ acting in the angular (momentum direction) space are defined as

$$
\hat{H}_{\mathrm{e}-\mathrm{h}}^{\alpha}(\omega, \boldsymbol{q})=\boldsymbol{v} \cdot \boldsymbol{q}-\frac{\omega}{1+\hat{F}^{\alpha}}
$$

and the velocity operator is

$$
\hat{\boldsymbol{s}}^{\alpha}(\omega, \boldsymbol{q})=\frac{\partial \hat{H}_{\mathrm{e}-\mathrm{h}}^{\alpha}(\omega, \boldsymbol{q})}{\partial \boldsymbol{q}}=\boldsymbol{v}+\omega \frac{\partial}{\partial \boldsymbol{q}}\left(\frac{\hat{F}^{\alpha}}{1+\hat{F}^{\alpha}}\right) .
$$

The action of the operators $\hat{F}^{\alpha}$ in the angular space is the same as in Eq. (3.3) and they have the following forms:

$$
\begin{aligned}
& \hat{F}^{g}=0 \\
& {\left[\hat{F}^{\sigma}\right]\left(\boldsymbol{n}_{i}, \boldsymbol{n}_{j}\right)=F^{\sigma}\left(\theta_{i j}\right)} \\
& {\left[\hat{F}^{\rho}\right]\left(\boldsymbol{n}_{i}, \boldsymbol{n}_{j}\right)=\nu V(\boldsymbol{q})+F^{\rho}\left(\theta_{i j}\right),}
\end{aligned}
$$

where $F^{\rho, \sigma}(\theta)$ are the Landau Fermi-liquid interaction parameters. The angular independent $\nu V(\boldsymbol{q})$ term takes into account the long range part of the Coulomb densitydensity interaction.

To characterize the density of states for the bosonic excitations we introduce the propagators $\hat{\mathcal{L}}^{\alpha}\left(\omega, \boldsymbol{q} ; \boldsymbol{n}_{i}, \boldsymbol{n}_{j}\right)$, $\alpha=\rho, \sigma, g$ as

$$
\left[i \hat{H}_{\mathrm{e}-\mathrm{h}}^{\alpha}(\omega, \boldsymbol{q})-\widehat{\mathrm{St}}_{\tau}\right] \hat{\mathcal{L}}^{\alpha}=\hat{1},
$$

They describe the propagation of the electron-hole pair which is scattered by the disorder potential. This propagation is affected by the corresponding interactions for $\alpha=\rho, \sigma$, whereas it reduces to the usual diffusion for the ghosts.

We are now prepared to write down the conservation laws which must be satisfied by the collision integrals independently of their explicit form or the particular shape of the distribution functions. The conservation of the number of particles is guarded by the condition

$$
\begin{aligned}
& \int \widehat{\mathrm{St}}^{e-\alpha}\left\{f, N^{\alpha}\right\}(\epsilon, \boldsymbol{n} ; t, \boldsymbol{r}) d \boldsymbol{n} d \epsilon=0 . \quad \alpha=g, \rho, \sigma \\
& \int \widehat{\mathrm{St}}^{e-e}\{f\}(\epsilon, \boldsymbol{n} ; t, \boldsymbol{r}) d \boldsymbol{n} d \epsilon=0,
\end{aligned}
$$

and the impurity collision integral (3.5) conserves the number of particles on each energy shell

$$
\int \widehat{\mathrm{St}}_{\tau} f(\epsilon, \boldsymbol{n} ; t, \boldsymbol{r}) d \boldsymbol{n}=0
$$

The conservation of energy during purely electron-electron collisions is dictated by

$$
\int \epsilon \widehat{\mathrm{St}}^{e-e}\{f\}(\epsilon, \boldsymbol{n} ; t, \boldsymbol{r}) d \boldsymbol{n} d \epsilon=0
$$

Finally, the conservation of energy during the electronboson collision is guaranteed by the conditions

$$
\begin{aligned}
& \nu \int \epsilon \widehat{\mathrm{St}}^{e-\alpha}\left\{f, N^{\alpha}\right\}(\epsilon, \boldsymbol{n} ; t, \boldsymbol{r}) \frac{d \boldsymbol{n} d \epsilon}{\Omega_{d}} \\
& +\int \operatorname{Tr}\left[\hat{\mathcal{L}}^{\alpha}(\omega) \widehat{\mathrm{St}}^{\alpha-e}\left\{f, N^{\alpha}\right\}(\omega ; t, \boldsymbol{r})\right] \frac{d \omega}{2 \pi} \\
& =-i \int \operatorname{Tr}\left[\left[\hat{H}_{\mathrm{e}-\mathrm{h}}^{\alpha}(\omega) ; \hat{\mathcal{L}}^{\alpha}(\omega)\right] \hat{N}^{\alpha}(\omega ; t, \boldsymbol{r})\right] \frac{\omega d \omega}{2 \pi}
\end{aligned}
$$

for $\alpha=g, \rho, \sigma$, where the trace is defined as

$$
\operatorname{Tr} \hat{A} \hat{B}=\int \frac{d \boldsymbol{n}_{1} d \boldsymbol{n}_{2}}{\Omega_{d}^{2}} \int \frac{d^{d} q}{(2 \pi)^{d}} A\left(\boldsymbol{q} ; \boldsymbol{n}_{1}, \boldsymbol{n}_{2}\right) B\left(\boldsymbol{q} ; \boldsymbol{n}_{2}, \boldsymbol{n}_{1}\right) .
$$

The existence of the conservation laws (3.12) immediately enables us to establish the expressions for the conserved currents in the spirit of Sec. II A. We find by 
integrating both sides of Eq. (3.4) over $\epsilon$ and $\boldsymbol{n}$ :

$$
\begin{aligned}
& \frac{\partial \rho}{\partial t}+\nabla \cdot \boldsymbol{j}=0 \\
& \rho(t, \boldsymbol{r})=e \nu \int f(\epsilon, \boldsymbol{n} ; t, \boldsymbol{r}) \frac{d \epsilon d \boldsymbol{n}}{\Omega_{d}} \\
& \boldsymbol{j}(t, \boldsymbol{r})=e \nu v_{F} \int \boldsymbol{n} f(\epsilon, \boldsymbol{n} ; t, \boldsymbol{r}) \frac{d \epsilon d \boldsymbol{n}}{\Omega_{d}}
\end{aligned}
$$

which express the conservation of charge in terms for the usual charge density and electric current density - cf. Eqs. (2.5)-(2.7).

Turning to the energy conservation, we multiply Eq. (3.4) by $\epsilon$ and then integrate over $\boldsymbol{n}, \epsilon$. Similarly, we pre-multiply Eq. (3.6) by $\hat{\mathcal{L}}^{\alpha}$, take the trace (3.13) and integrate over $\omega$. Adding the results together, one finds:

$$
\begin{aligned}
& \frac{\partial u_{t o t}}{\partial t}+\nabla \cdot \boldsymbol{j}_{t o t}^{\epsilon}=\boldsymbol{j} \cdot \boldsymbol{E} \\
& u_{t o t}=u_{e}+u_{\rho}+3 u_{\sigma}-4 u_{g} \\
& \boldsymbol{j}_{t o t}^{\epsilon}=\boldsymbol{j}_{e}^{\epsilon}+\boldsymbol{j}_{\rho}^{\epsilon}+3 \boldsymbol{j}_{\sigma}^{\epsilon}-4 \boldsymbol{j}_{g}^{\epsilon}
\end{aligned}
$$

The electronic contributions to the energy density and current density are given by

$$
\begin{aligned}
& u_{e}(t, \boldsymbol{r})=\nu \int \frac{d \epsilon d \boldsymbol{n}}{\Omega_{d}} \epsilon f(\epsilon, \boldsymbol{n} ; t, \boldsymbol{r}) \\
& \boldsymbol{j}_{e}^{\epsilon}(t, \boldsymbol{r})=\nu v_{F} \int \frac{d \epsilon d \boldsymbol{n}}{\Omega_{d}} \epsilon \boldsymbol{n} f(\epsilon, \boldsymbol{n} ; t, \boldsymbol{r}) .
\end{aligned}
$$

The contributions of the bosonic neutral excitations are

$$
\begin{aligned}
& u_{\alpha}(t, \boldsymbol{r})=\int \operatorname{Tr}\left\{\frac{1}{1+\hat{F}^{\alpha}} \hat{\mathcal{L}}^{\alpha}(\omega) \hat{N}^{\alpha}(\omega ; t, \boldsymbol{r})\right\} \frac{\omega d \omega}{2 \pi} \\
& j_{\alpha}^{\epsilon}(t, \boldsymbol{r})=\int \operatorname{Tr}\left\{\hat{\boldsymbol{s}}^{\alpha}(\omega) \hat{\mathcal{L}}^{\alpha}(\omega) \hat{N}^{\alpha}(\omega ; t, \boldsymbol{r})\right\} \frac{\omega d \omega}{2 \pi}
\end{aligned}
$$

for $\alpha=g, \rho, \sigma$.

Equations (3.14)-(3.15) constitute our main results: the conserved currents are defined in terms of the distribution functions of the quasiparticles which describe the low energy excitations of the interacting electron gas for interaction in the particle-hole channel. In contrast with previous calculations ${ }^{20-23}$, we explicitly show the validity of the continuity equation for the energy transport; no such proof has been presented before in the quantum kinetic equation approach ${ }^{38}$. Moreover we believe that the form of the energy current in those references is not correct, since it is not gauge invariant - see Appendix B for more details. As an additional benefit, our approach enables us to clearly identify the contributions of the collective modes and the scattering processes involved - this last task is accomplished by analysing the explicit form of the collision integrals, which is is also needed to calculate the transport coefficients. The detailed derivation of the collision integrals is presented in Sec. V; here we summarize the results and give them physical interpretation.

To shorten the formulas we introduce the following combinations of the distribution functions:

$$
\begin{aligned}
& \Upsilon_{i j ; k l}^{\alpha}(\epsilon, \omega, \boldsymbol{q} ; t, \boldsymbol{r}) \equiv N^{\alpha}\left(\omega, \boldsymbol{q} ; \boldsymbol{n}_{i}, \boldsymbol{n}_{j} ; t, \boldsymbol{r}\right) \\
& \quad \times\left\{f\left(\epsilon, \boldsymbol{n}_{k} ; t, \boldsymbol{r}\right)-f\left(\epsilon-\omega, \boldsymbol{n}_{k} ; t, \boldsymbol{r}\right)\right\} \\
& \quad+\Omega_{d} \delta\left(\widehat{\boldsymbol{n}_{i} \boldsymbol{n}_{j}}\right)\left\{f\left(\epsilon, \boldsymbol{n}_{l} ; t, \boldsymbol{r}\right)\left[1-f\left(\epsilon-\omega, \boldsymbol{n}_{k} ; t, \boldsymbol{r}\right)\right]\right\}
\end{aligned}
$$

which, as it follows from Eq. (3.2), has the property

$$
\int d \epsilon \Upsilon_{i j ; k l}^{\alpha}(\epsilon, \omega, \boldsymbol{q})=\int d \epsilon \Upsilon_{j i ; l k}^{\alpha}(\epsilon,-\omega,-\boldsymbol{q})
$$

and

$$
\begin{aligned}
& \Psi_{i j ; k l}\left(\epsilon, \epsilon_{1} ; \omega\right) \\
& \equiv f\left(\epsilon-\omega, \boldsymbol{n}_{i}\right)\left[1-f\left(\epsilon, \boldsymbol{n}_{j}\right)\right] f\left(\epsilon_{1}, \boldsymbol{n}_{k}\right)\left[1-f\left(\epsilon_{1}-\omega, \boldsymbol{n}_{l}\right)\right] \\
& -f\left(\epsilon, \boldsymbol{n}_{i}\right)\left[1-f\left(\epsilon-\omega, \boldsymbol{n}_{j}\right)\right] f\left(\epsilon_{1}-\omega, \boldsymbol{n}_{k}\right)\left[1-f\left(\epsilon_{1}, \boldsymbol{n}_{l}\right)\right] .
\end{aligned}
$$

It is easy to check that $\Upsilon=\Psi=0$ in the thermal equilibrium (3.1). The last combination enters the collision integral in the symmetric form

$$
\Psi_{i j}^{s} \equiv \frac{1}{4}\left[\Psi_{i j ; i j}+\Psi_{j i ; i j}+\Psi_{i j ; j i}+\Psi_{j i ; j i}\right]
$$

It is worth noticing that the terms involving four distribution functions $f$ are in fact cancelled from $\Psi^{s}$; moreover it has the properties:

$$
\begin{gathered}
\int \Psi_{i j}^{s}\left(\epsilon, \epsilon_{1} ; \omega\right) d \epsilon d \epsilon_{1}=0 \\
\int a(\omega) \int \epsilon \Psi_{i j}^{s}\left(\epsilon, \epsilon_{1} ; \omega\right) d \epsilon d \epsilon_{1} d \omega=0
\end{gathered}
$$

for any even function $a(\omega)$. Finally we introduce the vertex $\gamma$ for the impurity scattering

$$
\gamma_{i j}^{k} \equiv \frac{1}{\tau\left(\theta_{i j}\right)} \Omega_{d}\left[\delta\left(\widehat{\boldsymbol{n}_{j} \boldsymbol{n}_{k}}\right)-\delta\left(\widehat{\boldsymbol{n}_{i} \boldsymbol{n}_{k}}\right)\right]
$$

and the shorthand notation

$$
\mathcal{L}_{i j}^{\alpha} \equiv \mathcal{L}^{\alpha}\left(\omega, \boldsymbol{q} ; \boldsymbol{n}_{i}, \boldsymbol{n}_{j}\right)
$$

The explicit expression for the boson-electron collision integral is then

$$
\begin{aligned}
& \widehat{\mathrm{St}}^{\alpha-e}\left(\omega, \boldsymbol{q} ; \boldsymbol{n}_{1}, \boldsymbol{n}_{2} ; t, \boldsymbol{r}\right)=-\int d \epsilon \int \frac{d \boldsymbol{n}_{3} d \boldsymbol{n}_{4}}{\Omega_{d}^{2}} \\
& \quad \times\left\{\gamma_{14}^{3} \Upsilon_{32 ; 41}^{\alpha}(\epsilon, \omega, \boldsymbol{q} ; t, \boldsymbol{r})+\gamma_{34}^{2} \Upsilon_{13 ; 14}^{\alpha}(\epsilon, \omega, \boldsymbol{q} ; t, \boldsymbol{r})\right\},
\end{aligned}
$$

for $\alpha=g, \rho, \sigma$. The formula for the electron-boson collision integral can be conveniently decomposed into local $(l)$ and non-local $(n)$ [in the sense of Sec. II B] parts

$$
\widehat{\mathrm{St}}^{e-\alpha}=\widehat{\mathrm{St}}_{l}^{e-\alpha}+\widehat{\mathrm{St}}_{n}^{e-\alpha} \text {. }
$$




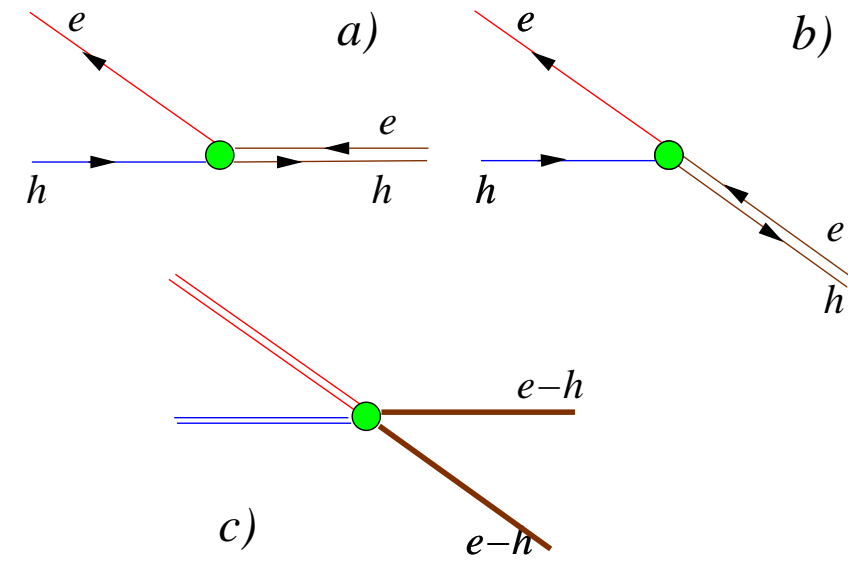

FIG. 2: The scattering amplitudes leading to the creation of the same electron and hole out of different electron hole pairs (double lines) (a-b) and their interference contribution (c). The impurity is denoted by filled circle.

The local part of the collision integral is

$$
\begin{aligned}
& \widehat{\mathrm{St}}_{l}^{e-\alpha}\left(\epsilon, \boldsymbol{n}_{1}\right)=\frac{1}{\nu} \int \frac{d \omega}{2 \pi} \frac{1}{\omega} \int \frac{d^{d} q}{(2 \pi)^{d}} \int \frac{d \boldsymbol{n}_{2} d \boldsymbol{n}_{3} d \boldsymbol{n}_{4}}{\Omega_{d}^{3}} \\
& \times\left\{\gamma_{12}^{3}\left[\mathcal{L}_{34}^{\alpha} \Upsilon_{41 ; 21}^{\alpha}(\epsilon, \omega, \boldsymbol{q})+\Upsilon_{34 ; 21}^{\alpha}(\epsilon, \omega, \boldsymbol{q}) \overline{\mathcal{L}}_{41}^{\alpha}\right]\right. \\
& \left.+\gamma_{21}^{3}\left[\mathcal{L}_{34}^{\alpha} \Upsilon_{42 ; 21}^{\alpha}(\epsilon, \omega, \boldsymbol{q})+\Upsilon_{34 ; 21}^{\alpha}(\epsilon, \omega, \boldsymbol{q}) \overline{\mathcal{L}}_{42}^{\alpha}\right]\right\}
\end{aligned}
$$

where the bar indicates Hermitian conjugation

$$
\overline{\mathcal{L}}^{\alpha}\left(\omega, \boldsymbol{q} ; \boldsymbol{n}_{i}, \boldsymbol{n}_{j}\right)=\mathcal{L}^{\alpha}\left(-\omega,-\boldsymbol{q} ; \boldsymbol{n}_{j}, \boldsymbol{n}_{i}\right)
$$

Using Eq. (3.11) and the definitions (3.13), (3.17) we can verify that the pair Eqs. (3.18)-(3.19b) satisfies the energy conservation law Eq. (3.12d) on its own.

Equation $(3.19 \mathrm{~b})$ satisfies the particle number conservation law (3.12a) as well. To check this, we make the change of variables $(\omega, \boldsymbol{q}) \rightarrow(-\omega,-\boldsymbol{q})$ in the terms containing $\overline{\mathcal{L}}^{\alpha}$ and then use Eqs. (3.16b) and (3.19c) to rewrite the integral of Eq. $(3.19 \mathrm{~b})$ in terms of $\mathcal{L}^{\alpha}$ only:

$$
\begin{aligned}
& \int{\widehat{\mathrm{St}_{l}}}_{l}^{e-\alpha}\left(\epsilon, \boldsymbol{n}_{1}\right) d \epsilon d \boldsymbol{n}_{1}=\ldots \int \frac{d \boldsymbol{n}_{1} \ldots d \boldsymbol{n}_{4}}{\Omega_{d}^{4}} \\
& \times\left\{\gamma_{12}^{3}\left[\mathcal{L}_{34}^{\alpha} \Upsilon_{41 ; 21}^{\alpha}(\epsilon, \omega, \boldsymbol{q})-\mathcal{L}_{14}^{\alpha} \Upsilon_{43 ; 12}^{\alpha}(\epsilon, \omega, \boldsymbol{q})\right]\right. \\
& \left.+\gamma_{21}^{3}\left[\mathcal{L}_{34}^{\alpha} \Upsilon_{42 ; 21}^{\alpha}(\epsilon, \omega, \boldsymbol{q})-\mathcal{L}_{24}^{\alpha} \Upsilon_{43 ; 12}^{\alpha}(\epsilon, \omega, \boldsymbol{q})\right]\right\}
\end{aligned}
$$

We perform the $\boldsymbol{n}_{3}$ integration using the delta functions in Eq. (3.17) and we obtain the result antisymmetric with respect to the $\boldsymbol{n}_{1} \leftrightarrow \boldsymbol{n}_{2}$ permutation. Hence the above expression vanishes after the $\boldsymbol{n}_{1,2}$ integrations.

The physical meaning of the collision integrals (3.18) and $(3.19 \mathrm{~b})$ is the following. In the absence of the disorder the electron-hole pair propagates for an infinitely long time. Due to the impurity potential the decay of the pair into the electron and hole moving in different directions as shown in Fig. 2 is allowed. Equations (3.18) and (3.19b) are the probabilities for such a decay. [See also Sec. VD after Eq. (5.67) for further discussion.]
The non-local contribution to the collision integral

$$
\begin{aligned}
& \widehat{\mathrm{St}}_{n}^{e-\alpha}\left(\epsilon, \boldsymbol{n}_{1}\right)=\frac{2}{\nu} \int \frac{d \omega}{2 \pi} \frac{1}{\omega^{2}} \int \frac{d^{d} q}{(2 \pi)^{d}} \int \frac{d \boldsymbol{n}_{2} \ldots d \boldsymbol{n}_{7}}{\Omega_{d}^{6}} \\
& \times \gamma_{13}^{2} \gamma_{46}^{5}\left[\mathcal{L}_{14}^{g}-\mathcal{L}_{34}^{g}\right] \sinh ^{2}\left(\frac{\omega \partial}{2 \partial \epsilon}\right)\left[f\left(\epsilon, \boldsymbol{n}_{6}\right)-f\left(\epsilon, \boldsymbol{n}_{4}\right)\right] \\
& \times \int d \epsilon_{1}\left\{\left[\Upsilon_{57 ; 64}^{\alpha}\left(\epsilon_{1}, \omega, \boldsymbol{q}\right)+\Upsilon_{57 ; 46}^{\alpha}\left(\epsilon_{1}, \omega, \boldsymbol{q}\right)\right] \overline{\mathcal{L}}_{72}^{\alpha}\right. \\
& \left.+\mathcal{L}_{57}^{\alpha}\left[\Upsilon_{72 ; 13}^{\alpha}\left(\epsilon_{1}, \omega, \boldsymbol{q}\right)+\Upsilon_{72 ; 31}^{\alpha}\left(\epsilon_{1}, \omega, \boldsymbol{q}\right)\right]\right\}
\end{aligned}
$$

by construction obeys its own conservation law

$$
\int \epsilon^{m} \widehat{\mathrm{St}}_{n}^{e-\alpha}\left(\epsilon, \boldsymbol{n}_{1}\right) d \epsilon=0, \quad m=0,1,
$$

i.e. it conserves the energy and the number of the electrons moving for a given momentum direction $\boldsymbol{n}$. Moreover, one can see that the collision integral (3.19d) does not contribute to the linear response at all because in the thermodynamic equilibrium $\Upsilon=0$ and $f$ does not depend on the angle. The non-locality of this collision integral indicates that the task formulated in Sec. IIB has not been quite accomplished. Technically, this nonlocality can be decoupled by introducing a density matrix non-diagonal in the boson-ghost space. We choose not to pursue this line because the term (3.19d) does not contribute to any observable quantity we are interested in and does not affect any conservation laws.

The electron-electron collision integral can be split into elastic, non-local and local parts:

$$
\widehat{\mathrm{St}}^{e-e}\left(\epsilon, \boldsymbol{n}_{1}\right)={\widehat{\mathrm{St}^{e l}}}_{e l}^{-e}\left(\epsilon, \boldsymbol{n}_{1}\right)+{\widehat{\mathrm{St}_{n}}}^{e-e}\left(\epsilon, \boldsymbol{n}_{1}\right)+{\widehat{\mathrm{St}_{l}}}_{l}^{e-e}\left(\epsilon, \boldsymbol{n}_{1}\right) \text {. }
$$

The elastic term describes the scattering of the electron on the static self-consistent potential created by all the other electrons

$$
\begin{aligned}
& \widehat{\mathrm{St}}_{e l}^{e-e}=\frac{2}{\nu} \operatorname{Re} \int \frac{d \omega}{2 \pi} \frac{1}{\omega} \int \frac{d^{d} q}{(2 \pi)^{d}} \int \frac{d \boldsymbol{n}_{2} \ldots d \boldsymbol{n}_{6}}{\Omega_{d}^{5}} \gamma_{13}^{2} \gamma_{46}^{5} \\
& \quad \times\left[\mathcal{L}^{\rho}+3 \mathcal{L}^{\sigma}-4 \mathcal{L}^{g}\right]_{52}\left[f\left(\epsilon-\omega, \boldsymbol{n}_{6}\right)-f\left(\epsilon-\omega, \boldsymbol{n}_{4}\right)\right] \\
& \quad \times\left[\mathcal{L}_{14}^{g} f\left(\epsilon, \boldsymbol{n}_{3}\right)+\mathcal{L}_{34}^{g} f\left(\epsilon, \boldsymbol{n}_{1}\right)\right] .
\end{aligned}
$$

Its physical origin is discussed in details in Ref. 17. Being elastic, it conserves the number of particle for each energy shell:

$$
\int \widehat{\operatorname{St}}_{e l}^{e-e}\left(\epsilon, \boldsymbol{n}_{1}\right) d \boldsymbol{n}_{1}=0,
$$

as one can see from the property $\gamma_{13}^{2}=-\gamma_{31}^{2}$ of the vertex (3.17).

The nonlocal term

$$
\begin{aligned}
& \widehat{\mathrm{St}}_{n}^{e-e}=-\frac{4}{\nu} \operatorname{Re} \int \frac{d \omega}{2 \pi} \frac{1}{\omega^{2}} \int \frac{d^{d} q}{(2 \pi)^{d}} \int \frac{d \boldsymbol{n}_{2} \ldots d \boldsymbol{n}_{6}}{\Omega_{d}^{5}} \gamma_{13}^{2} \gamma_{46}^{5} \\
& \times \int d \epsilon_{1}\left\{\left[\mathcal{L}_{14}^{g}-\mathcal{L}_{34}^{g}\right]\left[\overline{\mathcal{L}}^{\rho}+3 \overline{\mathcal{L}}^{\sigma}-4 \overline{\mathcal{L}}^{g}\right]_{52} \Psi_{46}^{s}\left(\epsilon, \epsilon_{1}, ; \omega\right)\right.
\end{aligned}
$$


describes the inelastic electron-electron collisions during which bosons and ghosts act as virtual states. [The function $\Psi^{s}$ were introduced in Eq. (3.16d)]. The real part being an even function, we can use Eq. (3.16e) to verify that Eq. (3.20d) obeys the conservation law

$$
\int \epsilon^{m} \widehat{\mathrm{St}}_{n, l}^{e-e}\left(\epsilon, \boldsymbol{n}_{1}\right) d \epsilon=0, \quad m=0,1
$$

As indicated, the same law is satisfied by the local (and elastic) term:

$$
\begin{aligned}
& \widehat{\mathrm{St}}_{l}^{e-e}=\frac{2}{\nu} \int \frac{d \omega}{2 \pi} \frac{1}{\omega^{2}} \int \frac{d^{d} q}{(2 \pi)^{d}} \int \frac{d \boldsymbol{n}_{2} \ldots d \boldsymbol{n}_{6}}{\Omega_{d}^{5}} \int d \epsilon_{1} \gamma_{13}^{2} \gamma_{46}^{5} \\
& \times\left[\mathcal{L}_{14}^{g}+\mathcal{L}_{34}^{g}\right]\left[\mathcal{L}^{\rho}+3 \mathcal{L}^{\sigma}-4 \mathcal{L}^{g}\right]_{52} \\
& \times \sinh ^{2}\left(\frac{\omega \partial}{2 \partial \epsilon}\right)\left[f\left(\epsilon, \boldsymbol{n}_{6}\right)-f\left(\epsilon, \boldsymbol{n}_{4}\right)\right] \\
& \times\left[f\left(\epsilon_{1}, \boldsymbol{n}_{1}\right)\left[1-f\left(\epsilon_{1}-\omega, \boldsymbol{n}_{3}\right)\right]+\left(\boldsymbol{n}_{1} \leftrightarrow \boldsymbol{n}_{3}\right)\right] .
\end{aligned}
$$

Therefore equation (3.20e) enables us to conclude that both collision integrals (3.20d) and (3.20f) do not affect the transport coefficients [when they can be considered as perturbations in comparison to the bare impurity collision integral].

We note that, although it might not be evident, the present form of the kinetic equation permits the proper identification of the inelastic kernel that determines the phase relaxation time - further details can be found in Appendix G.

\section{SUMMARY OF THE RESULTS FOR THERMAL TRANSPORT AND SPECIFIC HEAT}

In this section we present our final answers for the interaction corrections to the thermal conductivity and the specific heat. They are obtained by solving the kinetic equations and then substituting the solutions into the definitions (3.15) for the energy and energy current densities. The explicit calculations are performed in Sec. VI. We will consider the short range impurities $\tau(\theta)=\tau$. We report our results for quasi one-dimensional and threedimensional systems in the diffusive limit $T \tau \ll \hbar$; for two-dimensional systems, we will not put such a restriction on the temperature range. However common to all dimensionality is the zeroth harmonic approximation for the Fermi liquid constants [see Eq. (6.14)].

\section{A. Thermal conductivity}

In the absence of the magnetic field the thermal conductivity tensor is diagonal, $\kappa_{\mu \nu}=\kappa \delta_{\mu \nu}$, and we will write the expression for the diagonal components only as:

$$
\kappa=\kappa_{W F}+\Delta \kappa
$$

The first term is given by the Wiedemann-Franz law $\kappa_{W F}=L \sigma T$ with the inclusion of the interaction corrections to the conductivity and the Lorentz number given in Eq. (1.1). The second term causes a violation of the Wiedemann-Franz law. In the diffusive limit and for low dimensionality the main contribution to $\Delta \kappa$ is due to the long range nature of the bosonic energy transport, which originates from the long range part of the interaction in the singlet channel. In the quasi ballistic case a large contribution comes from the inelastic scattering of the electron on the bosons as well. Smaller corrections arise due to the triplet channel bosonic transport and to the energy dependence of the elastic scattering.

For quasi one-dimensional and three-dimensional systems in the diffusive limit we write

$$
\Delta \kappa=\delta \kappa^{\rho}+3 \delta \kappa^{\sigma}+\delta \kappa_{e l}
$$

where the bosonic corrections include the ghost contributions

$$
\delta \kappa^{\alpha}=\kappa^{\alpha}-\kappa^{g}, \alpha=\rho, \sigma
$$

[see Eq. (6.12) for the definition of $\kappa^{\alpha}$ ] and we neglect the inelastic contributions $\delta \kappa_{i n}$ which are smaller by the parameter $T \tau / \hbar$.

For quasi one-dimensional systems the explicit expressions are:

$$
\begin{aligned}
\delta \kappa_{e l}= & \frac{1}{8 \sqrt{2 \pi}} \zeta\left(\frac{3}{2}\right) \sqrt{\frac{D T}{\hbar}} \\
& \times\left\{-1+3\left[1-\frac{2}{F_{0}^{\sigma}}\left(1+F_{0}^{\sigma}-\sqrt{1+F_{0}^{\sigma}}\right)\right]\right\} \\
\delta \kappa^{\rho}= & \frac{3}{8 \sqrt{2 \pi}} \zeta\left(\frac{3}{2}\right) \sqrt{\frac{D T}{\hbar}} a k \ln ^{\frac{1}{2}}\left(\frac{\hbar D k^{2}}{T}\right) \\
\delta \kappa^{\sigma}= & \frac{3}{8 \sqrt{2 \pi}} \zeta\left(\frac{3}{2}\right) \sqrt{\frac{D T}{\hbar}}\left[\sqrt{1+F_{0}^{\sigma}}-1\right]
\end{aligned}
$$

where $a$ is a length of the order of the wire width and $k=\sqrt{4 \pi e^{2} \nu}$ is the inverse screening length in the bulk.

For three-dimensional systems the results are:

$$
\begin{aligned}
\delta \kappa_{e l}= & \frac{5}{48 \sqrt{2 \pi^{3}}} \zeta\left(\frac{5}{2}\right) \sqrt{\frac{T^{3}}{\hbar^{3} D}} \\
& \times\left\{1+3\left[1-\frac{2}{F_{0}^{\sigma}}\left(1-\frac{1}{\sqrt{1+F_{0}^{\sigma}}}\right)\right]\right\} \\
\delta \kappa^{\rho}= & \frac{15}{32 \sqrt{2 \pi^{3}}} \zeta\left(\frac{5}{2}\right) \sqrt{\frac{T^{3}}{\hbar^{3} D}} \\
\delta \kappa^{\sigma}= & \frac{15}{32 \sqrt{2 \pi^{3}}} \zeta\left(\frac{5}{2}\right) \sqrt{\frac{T^{3}}{\hbar^{3} D}}\left[1-\frac{1}{\sqrt{1+F_{0}^{\sigma}}}\right]
\end{aligned}
$$

In these expressions, $\zeta(x)$ is the Riemann zeta function and $\zeta(3 / 2) \approx 2.612, \zeta(5 / 2) \approx 1.341$.

For two-dimensional systems, we separate the corrections due to the singlet and the triplet channel interactions:

$$
\Delta \kappa=\Delta \kappa_{s}+3 \Delta \kappa_{t}
$$


The singlet channel contribution is, with logarithmic accuracy:

$$
\begin{aligned}
\Delta \kappa_{s}= & \frac{T}{6 \hbar} g_{1}\left(2 \pi \frac{T \tau}{\hbar}\right) \ln \left(\frac{\hbar v_{F} k}{T}\right) \\
& -\frac{T}{24 \hbar} g_{2}\left(\pi \frac{T \tau}{\hbar}\right) \ln \left(1+\frac{\hbar^{2}}{(T \tau)^{2}}\right) \\
& -\frac{\pi^{2}}{15} \frac{T}{\hbar}\left(\frac{T \tau}{\hbar}\right)^{2} \ln \left(\frac{E_{F}}{T}\right)
\end{aligned}
$$

where $k=2 \pi e^{2} \nu$ is the two-dimensional inverse screening length. The cross-over functions $g_{1}$ and $g_{2}$ are given in Eq. (6.42). Here we note that $g_{1}(x), g_{2}(x) \simeq 1$ for $x \ll 1$ and $g_{1}(x) \simeq 3 / x, g_{2}(x) \simeq 14 x^{2} / 15$ for $x \gg 1$.

For the triplet channel we have:

$$
\Delta \kappa_{t}=\left\{\begin{array}{cc}
-\frac{T}{18 \hbar}\left[1-\frac{1}{F_{0}^{\sigma}} \ln \left(1+F_{0}^{\sigma}\right)\right] & \\
+\frac{T}{12 \hbar} \ln \left(1+F_{0}^{\sigma}\right) & , T \tau \ll 1 \\
-\frac{\pi^{2}}{15} \frac{T}{\hbar}\left(\frac{T \tau}{\hbar}\right)^{2} \ln \left(\frac{E_{F}}{T}\right)\left(\frac{F_{0}^{\sigma}}{1+F_{0}^{\sigma}}\right)^{2} & , T \tau \gg 1
\end{array}\right.
$$

In the diffusive limit $T \tau \ll \hbar$ our results are consistent with those of Ref. 22, even though the form of the energy current operator used in this reference is, to our opinion, incorrect - see Appendix B.

\section{B. Specific heat}

The specific heat is given by

$$
C_{V}=\frac{\pi^{2}}{3} \nu T+\delta C_{V}
$$

where the first term is the usual non-interacting electronic contribution and the second term is the bosonic interaction correction one.

For quasi one-dimensional and three-dimensional systems

$$
\delta C_{V}=\left(1+3 \Lambda_{d}\right)\left(\frac{T}{\hbar D}\right)^{\frac{d}{2}} a_{d}
$$

The two terms in the first bracket are respectively the singlet and triplet channel contributions. The singlet channel term is considered in the unitary limit and is therefore independent of any interaction parameter. On the other hand, the Fermi liquid parameter for the interaction in the triplet channel enter into Eq. (4.6) as

$$
\Lambda_{d}=1-\frac{1}{\left(1+F_{0}^{\sigma}\right)^{d / 2}}
$$

and the numerical factors $a_{1,3}$ are:

$$
\begin{aligned}
a_{3} & =\frac{15}{32 \pi \sqrt{2 \pi}} \zeta\left(\frac{5}{2}\right) \\
a_{1} & =-\frac{3}{8 \sqrt{2 \pi}} \zeta\left(\frac{3}{2}\right)
\end{aligned}
$$

For two-dimensional systems the result is, with logarithmic accuracy:

$$
\begin{aligned}
\delta C_{V}=-\left(\frac{T}{\hbar D}\right)[ & \left(1+3 \Lambda_{2}\right) \frac{1}{12} \ln \left(\frac{E_{F}}{T}\right) \\
& \left.+\left(1+3 \Lambda_{2}^{2}\right) \frac{3}{4 \pi} \zeta(3) T \tau\right]
\end{aligned}
$$

where $\zeta(3) \approx 1.202$. The first term on the right hand side extends to higher temperatures the logarithmic behaviour known in the diffusive limit (the upper cutoff is of the order of the Fermi energy $E_{F}$ and not $\hbar / \tau$ ); the second term becomes relevant in the quasi ballistic limit. In the diffusive limit our results are the same as those obtained in Ref. 12 by explicit thermodynamic calculation.

\section{DERIVATION OF THE KINETIC EQUATION}

This section is devoted to the derivation of the local kinetic equation. We first introduce the Eilenberger equation and some basic notation. Then we perform a (generalized) gauge transformation: this is the crucial step that enable us to obtain the local description. Then we introduce the bosonic degrees of freedom and derive the collision integrals.

\section{A. Eilenberger equation}

Our starting point for the derivation of the kinetic equation is the same as in Ref. 17 and we briefly summarize it here.

The interaction with small momentum and energy transfer in the singlet channel [the triplet channel will be discussed in section $\mathrm{VH}]$ is decoupled using the two Hubbard-Stratonovich fields $\phi_{ \pm}(t, \boldsymbol{r}, \boldsymbol{n})$. For the purpose of the one loop approximation we will employ, these fields can be considered as Gaussian with the propagators

$$
\begin{aligned}
\left\langle\left\langle\phi_{+}(1) \phi_{+}(2)\right\rangle\right\rangle & =-\frac{i}{2} D^{K}(1,2), \\
\left\langle\left\langle\phi_{+}(1) \phi_{-}(2)\right\rangle\right\rangle & =-\frac{i}{2} D^{R}(1,2), \\
\left\langle\left\langle\phi_{-}(1) \phi_{+}(2)\right\rangle\right\rangle & =-\frac{i}{2} D^{A}(1,2), \\
\left\langle\left\langle\phi_{-}(1) \phi_{-}(2)\right\rangle\right\rangle & =0 .
\end{aligned}
$$

where $\langle\langle\ldots\rangle\rangle$ means averaging over the fields $\phi_{ \pm}$. We used the short hand notation

$$
\begin{aligned}
& (i) \equiv\left(t_{i}, \boldsymbol{r}_{i}, \boldsymbol{n}_{i}\right), \int d i \equiv \int d t_{i} d \boldsymbol{r}_{i} \int \frac{d \boldsymbol{n}_{i}}{\Omega_{d}}, \\
& \left(i^{*}\right) \equiv\left(\boldsymbol{r}_{i}, \boldsymbol{n}_{i}\right), \int d i^{*} \equiv \int d \boldsymbol{r}_{i} \int \frac{d \boldsymbol{n}_{i}}{\Omega_{d}}
\end{aligned}
$$

where $i=1,2, \ldots$ and $\Omega_{d}$ is the total solid angle. 
We introduce the disorder averaged Green's function of the electron in the field $\phi_{ \pm}$in its matrix form in Keldysh space:

$$
\widehat{G}(1,2 \mid \phi)=\left(\begin{array}{ll}
G^{R}(1,2 \mid \phi) & G^{K}(1,2 \mid \phi) \\
G^{Z}(1,2 \mid \phi) & G^{A}(1,2 \mid \phi)
\end{array}\right)_{K}
$$

such that its average over the fluctuating field $\phi_{ \pm}$gives the usual expressions for the physical propagators:

$$
\begin{aligned}
& \left\langle\left\langle G^{R}(1,2)\right\rangle\right\rangle=-i \theta\left(t_{1}-t_{2}\right)\left\langle\psi(1) \psi^{\dagger}(2)+\psi^{\dagger}(2) \psi(1)\right\rangle, \\
& \left\langle\left\langle G^{A}(1,2)\right\rangle\right\rangle=i \theta\left(t_{2}-t_{1}\right)\left\langle\psi(1) \psi^{\dagger}(2)+\psi^{\dagger}(2) \psi(1)\right\rangle, \\
& \left\langle\left\langle G^{K}(1,2)\right\rangle\right\rangle=-i\left\langle\psi(1) \psi^{\dagger}(2)-\psi^{\dagger}(2) \psi(1)\right\rangle, \\
& \left\langle\left\langle G^{Z}(1,2)\right\rangle\right\rangle=0 .
\end{aligned}
$$

Here $\theta(t)$ is the Heaviside step function, $\psi^{\dagger}, \psi$ are the fermionic creation/annihilation operators in the Heisenberg representation and quantum mechanical averaging $\langle\ldots\rangle$ is performed with an arbitrary distribution function to be found from the solution of the kinetic equation.

For the disorder averaged Green's function, the semiclassical approximation is obtained by integrating the Wigner transform of $\widehat{G}(1,2 \mid \phi)$ over the distance from the Fermi surface:

$$
\begin{gathered}
\widehat{G}\left(t_{1}, t_{2}, \boldsymbol{p}, \boldsymbol{R}\right)=\int d^{2} r e^{i \boldsymbol{P} \cdot \boldsymbol{r}} \widehat{G}(1,2 \mid \phi) \\
\boldsymbol{r}=\boldsymbol{r}_{1}-\boldsymbol{r}_{2} ; \quad \boldsymbol{R}=\frac{1}{2}\left(\boldsymbol{r}_{1}+\boldsymbol{r}_{2}\right) \\
\boldsymbol{P}=\boldsymbol{p}-\frac{1}{2}\left[\mathbf{A}\left(t_{1}, \boldsymbol{R}\right)+\mathbf{A}\left(t_{2}, \boldsymbol{R}\right)\right] \\
\hat{g}\left(t_{1}, t_{2}, \boldsymbol{n}, \boldsymbol{r}\right)=\frac{i}{\pi} \int_{-\infty}^{\infty} d \xi \widehat{G}\left(t_{1}, t_{2}, \boldsymbol{n}\left[p_{F}+\frac{\xi}{v_{F}}\right], \boldsymbol{r}\right)
\end{gathered}
$$

where $\mathbf{A}$ is the vector potential of an external electromagnetic field, $p_{F}$ the Fermi momentum and $v_{F}$ the Fermi velocity. The dynamics of the semiclassical Green's function $\hat{g}$ in the matrix form is governed by the Eilenberger equation $^{24}$ :

$$
\left[\tilde{\partial}_{t}+\boldsymbol{v} \cdot \tilde{\nabla}+\boldsymbol{\omega}_{c} \cdot\left(\boldsymbol{n} \times \frac{\partial}{\partial \boldsymbol{n}}\right)\right] \hat{g}+i[\hat{\phi} ; \hat{g}]=\frac{\left[\hat{g} \circ \widehat{\mathrm{St}}_{\tau} \hat{g}\right]}{2}
$$

where $\boldsymbol{v}=v_{F} \boldsymbol{n}$, the action of the "bare" collision integral on any function $a(\boldsymbol{n})$ is defined as

$$
\begin{aligned}
{\left[\widehat{\mathrm{St}}_{\tau} a\right](\boldsymbol{n}) } & =\int \frac{d \boldsymbol{n}_{1}}{\Omega_{d}} \mathrm{St}_{\tau}\left(\boldsymbol{n}, \boldsymbol{n}_{1}\right) a\left(\boldsymbol{n}_{1}\right), \\
\mathrm{St}_{\tau}\left(\boldsymbol{n}_{1}, \boldsymbol{n}_{2}\right) & =\frac{1}{\tau\left(\theta_{12}\right)}-\delta\left(\widehat{\boldsymbol{n}_{1} \boldsymbol{n}_{2}}\right) \int \frac{d \boldsymbol{n}_{2}}{\tau\left(\theta_{12}\right)}
\end{aligned}
$$

and $\theta_{12}=\widehat{\boldsymbol{n}_{1} \boldsymbol{n}_{2}}$ [for the short-range impurity $\tau(\theta)$ does not depend on $\theta$; however, the formulas derived here will be valid for the arbitrary impurity scattering]. The time convolution of two matrices $\hat{a}\left(t_{1}, t_{2}\right)$ and $\hat{b}\left(t_{1}, t_{2}\right)$ is:

$\hat{a} \circ \hat{b}=\int d t_{3} \hat{a}\left(t_{1}, t_{3}\right) \hat{b}\left(t_{3}, t_{2}\right) ; \quad[\hat{a} \circ \hat{b}]=\hat{a} \circ \hat{b}-\hat{b} \circ \hat{a}$.

Defining the commutator between a matrix $\hat{c}(t, \boldsymbol{r}, \boldsymbol{n})$ and $\hat{g}$ as

$$
[\hat{c} ; \hat{g}]=\hat{c}\left(t_{1}, \boldsymbol{r}, \boldsymbol{n}\right) \hat{g}\left(t_{1}, t_{2}, \boldsymbol{n}, \boldsymbol{r}\right)-\hat{g}\left(t_{1}, t_{2}, \boldsymbol{n}, \boldsymbol{r}\right) \hat{c}\left(t_{2}, \boldsymbol{r}, \boldsymbol{n}\right)
$$

the covariant derivatives in Eq. (5.7) are given by:

$$
\begin{aligned}
\tilde{\partial}_{t} \hat{g} & =\partial_{t_{1}} \hat{g}+\partial_{t_{2}} \hat{g}+i[\hat{\varphi} ; \hat{g}] \\
\tilde{\nabla} \hat{g} & =\nabla \hat{g}+i[\hat{\mathbf{A}} ; \hat{g}]
\end{aligned}
$$

with $\hat{\mathbf{A}}=\mathbf{A} \hat{\mathbb{1}}_{K}$ and $\hat{\varphi}=\varphi \hat{\mathbb{1}}_{K}$. Here $\hat{\mathbb{1}}_{K}$ denotes the unit matrix in Keldysh space and $\varphi$ is the scalar potential for an external electromagnetic field such that:

$$
e \boldsymbol{E}=-\boldsymbol{\nabla} \varphi+\partial_{t} \mathbf{A} \quad, \quad e \boldsymbol{B}=-c \boldsymbol{\nabla} \times \mathbf{A}
$$

The vector $\boldsymbol{\omega}_{c}=e \boldsymbol{B} /(m c)$ has the magnitude of the cyclotron frequency and the direction of the magnetic field $\boldsymbol{B}$. Finally, $\hat{\phi}$ is the matrix in the Keldysh space:

$$
\hat{\phi}=\left(\begin{array}{ll}
\phi_{+} & \phi_{-} \\
\phi_{-} & \phi_{+}
\end{array}\right)_{K}
$$

The matrix Green's function $\hat{g}$ is subject to the following constraints:

$$
\begin{aligned}
& \hat{g}(\boldsymbol{n}, \boldsymbol{r}) \circ \hat{g}(\boldsymbol{n}, \boldsymbol{r})=\delta\left(t_{1}-t_{2}\right) \hat{\mathbb{1}}_{K} \\
& \int d t \operatorname{Tr} \hat{g}(t, t, \boldsymbol{n}, \boldsymbol{r})=0 .
\end{aligned}
$$

In thermal equilibrium, the relation

$$
\begin{aligned}
& g^{K}\left(t_{1}, t_{2}\right)=\left[g^{R} \circ n-n \circ g^{A}\right]\left(t_{1}, t_{2}\right) \\
& n\left(t_{1}, t_{2}\right)=\int \frac{d \epsilon}{2 \pi} e^{i \epsilon\left(t_{2}-t_{1}\right)} n(\epsilon) \\
& n(\epsilon)=1-2 f_{F}(\epsilon)=2 \tanh \frac{\epsilon}{2 T}
\end{aligned}
$$

must hold independently of the form of the spectral functions $g^{R, A}$.

In what follows we will assume that there is no magnetic field, $\boldsymbol{B}=0$ and $\boldsymbol{\omega}_{c}=0$, but no gauge choice is made: although one could set $\mathbf{A}=0$ by a gauge transformation, both the scalar and vector external potentials are left arbitrary in order to keep track of the gauge invariance of the equations.

As for the propagators defined by Eq. (5.1), they satisfy matrix Dyson's equation:

$$
\begin{aligned}
& \hat{D}(1,2)=\hat{D}_{0}(1,2)+\int d 3 \int d 4 \hat{D}_{0}(1,3) \hat{\Pi}(3,4) \hat{D}(4,2) ; \\
& \hat{D}_{0}(1,2)=-\left[V\left(\boldsymbol{r}_{12}\right)+\frac{F^{\rho}\left(\theta_{12}\right) \delta\left(\boldsymbol{r}_{12}\right)}{\nu}\right] \delta\left(t_{12}\right) \hat{\mathbb{1}}_{K} ;
\end{aligned}
$$


where $V(\boldsymbol{r})$ is the long range part of the interaction (for the Coulomb interaction $\left.V(\boldsymbol{r})=e^{2} /|\boldsymbol{r}|\right), \theta_{12}=\widehat{\boldsymbol{n}_{1} \boldsymbol{n}_{2}}$, $\boldsymbol{r}_{12}=\boldsymbol{r}_{1}-\boldsymbol{r}_{2}, t_{12}=t_{1}-t_{2}$. The matrix propagator is denoted by $\hat{D}$ and $\hat{\Pi}$ is the matrix polarization operator. They have a structure similar to the Green's function one:

$$
\hat{D}=\left(\begin{array}{cc}
D^{R} & D^{K} \\
0 & D^{A}
\end{array}\right)_{K}, \quad \hat{\Pi}=\left(\begin{array}{cc}
\Pi^{R} & \Pi^{K} \\
0 & \Pi^{A}
\end{array}\right)_{K}
$$

The polarization operators are given by variational derivatives of the solutions to the Eilenberger equation $(5.7)$ :

$$
\begin{gathered}
\Pi^{R}(1,2)=\Pi^{A}(2,1)=\nu\left[\delta_{12}+\frac{\pi}{2} \frac{\delta g^{K}\left(t_{1}, t_{1}, \boldsymbol{n}_{1}, \boldsymbol{r}_{1}\right)}{\delta \phi_{+}\left(t_{2}, \boldsymbol{r}_{2}, \boldsymbol{n}_{2}\right)}\right] \\
\Pi^{K}(1,2)=\frac{\pi \nu}{2}\left[\frac{\delta\left(g^{K}+g^{Z}\right)\left(t_{1}, t_{1}, \boldsymbol{n}_{1}, \boldsymbol{r}_{1}\right)}{\delta \phi_{-}\left(t_{2}, \boldsymbol{r}_{2}, \boldsymbol{n}_{2}\right)}\right],
\end{gathered}
$$

where

$$
\delta_{12} \equiv \Omega_{d} \delta\left(\widehat{\boldsymbol{n}_{1} \boldsymbol{n}_{2}}\right) \delta\left(\boldsymbol{r}_{1}-\boldsymbol{r}_{2}\right) \delta\left(t_{1}-t_{2}\right)
$$

with $\Omega_{d}$ being the total solid angle.

\section{B. The gauge transformation}

With the Eilenberger equation (5.7) at hand, one could proceed as in Ref. 17 in order to derive an equation for the distribution function. However the resulting inelastic part of the collision integral, expressed in terms of the electron distribution function only, is non-local and the evaluation of e.g. the thermal conductivity would require the time and spatial gradient expansion of this term in the spirit of Eq. (2.15). As we already discussed, such a route makes the energy conservation in the kinetic equation obscure. Here we follow a different approach, inspired by the following considerations ${ }^{25}$ : if the fluctuating fields were uniform, they could be eliminated from Eq. (5.7) by a gauge transformation

$$
\hat{g} \rightarrow e^{-i \int t_{1} \hat{\phi}(t) d t} \hat{g} e^{i \int^{t_{2}} \hat{\phi}(t) d t} .
$$

In other words, the position-independent fluctuations of the $\phi$ fields define the time dependent position of the energy levels but the occupation numbers for such levels do not change. Therefore such fluctuations affect neither the electric transport nor the electron contribution to the thermal transport in the system. Moreover, if the path of the electron were a straight line, all the smooth fluctuating fields could still be eliminated in the eikonal approximation and once again they should not affect the electronic contribution to the transport. To get rid of such spurious contributions we will employ the gauge transformation described below.
We introduce a new matrix field $\hat{K}(t, \boldsymbol{n}, \boldsymbol{r})$ :

$$
\hat{K}=\left(\begin{array}{ll}
K_{+} & K_{-} \\
K_{-} & K_{+}
\end{array}\right)
$$

which is a functional of the field $\hat{\phi}$ and is used to perform the "generalized" gauge transformation

$$
\hat{g} \rightarrow e^{-i \hat{K}\left(t_{1}, \boldsymbol{n}, \boldsymbol{r}\right)} \hat{g} e^{i \hat{K}\left(t_{2}, \boldsymbol{n}, \boldsymbol{r}\right)} .
$$

This transformation is unitary and as such preserves the constraints (5.13). As we will see, it will lead us to the local kinetic equations. Applying the transformation to the Eilenberger equation (5.7), we obtain

$$
\left[\tilde{\partial}_{t}+\boldsymbol{v} \cdot \tilde{\nabla}\right] \hat{g}-i\left[\left(\partial_{t}+\boldsymbol{v} \cdot \boldsymbol{\nabla}\right) \hat{K}-\hat{\phi}, \hat{g}\right]=\frac{1}{2}\left[\hat{g} \circ \widehat{\mathrm{St}}_{\tau}^{\phi} \hat{g}\right],
$$

where

$$
\begin{aligned}
& {\left[\widehat{\mathrm{St}}_{\tau}^{\phi} \hat{g}\right]\left(t_{1}, t_{2}, \boldsymbol{n}\right) \equiv \int \frac{d \boldsymbol{n}_{1}}{\Omega_{d}} \mathrm{St}_{\tau}\left(\boldsymbol{n}, \boldsymbol{n}_{1}\right)} \\
& \times e^{i \hat{K}\left(t_{1}, \boldsymbol{n}\right)} e^{-i \hat{K}\left(t_{1}, \boldsymbol{n}_{1}\right)} \hat{g}\left(t_{1}, t_{2}, \boldsymbol{n}_{1}\right) e^{i \hat{K}\left(t_{2}, \boldsymbol{n}_{1}\right)} e^{-i \hat{K}\left(t_{2}, \boldsymbol{n}\right)} .
\end{aligned}
$$

The "bare" impurity collision integral and the derivatives are defined respectively in Eq. (5.8) and (5.11). We suppressed the $\boldsymbol{r}$ argument which is the same in all functions.

We will look for a perturbative solution to the Eilenberger equation in the form (5.22) in the first loop approximation; to do so it will suffice to retain only the terms at most quadratic in the $K$ fields in the collision integral. At the lowest order, $\hat{g}$ has the form:

$$
\hat{g}=\left(\begin{array}{cc}
\delta\left(t_{1}-t_{2}\right) & g^{K} \\
0 & -\delta\left(t_{1}-t_{2}\right)
\end{array}\right) .
$$

We will require that such form is preserved even in the first and second order in $K$, i.e. the corrections to the spectrum (described by $g^{R, A}$ ) are indeed eliminated by the gauge transformation.

At the linear order, the retarded, advanced and ' $\mathrm{Z}$ ' components of Eq. (5.22) vanish if $K_{-}$satisfies the equation

$$
\left(\partial_{t}+\boldsymbol{v} \cdot \nabla\right) K_{-}+\widehat{\mathrm{St}}_{\tau} K_{-}=\phi_{-}
$$

The solution to the integro-differential equation (5.24) can be written in terms of the diffuson propagator $\mathcal{L}^{g}\left(t_{1}, t_{2} ; \boldsymbol{n}_{1}, \boldsymbol{n}_{2} ; \boldsymbol{r}_{1}, \boldsymbol{r}_{2}\right)$ - the retarded solution to the classical kinetic equation

$$
\left(\partial_{t_{1}}+\boldsymbol{v}_{1} \cdot \nabla_{\boldsymbol{r}_{1}}-\widehat{\mathrm{St}}_{\tau}\right) \mathcal{L}^{g}=\delta_{12}
$$

where $\delta_{12}$ is defined in Eq. (5.18). Using Eq. (5.25), we find

$$
K_{-}(1)=-\int d 2 \overline{\mathcal{L}}^{g}(1,2) \phi_{-}(2), \quad \overline{\mathcal{L}}^{g}(1,2)=\mathcal{L}^{g}(2,1),
$$


where we use the shorthand notation (5.2). In an operator notation, Eq. (5.26) can be rewritten as

$$
K_{-}=-\hat{\overline{\mathcal{L}}}^{g} \phi_{-} .
$$

To simplify further manipulations, we introduce the following function of three angular variables

$$
\gamma\left(\begin{array}{c}
\boldsymbol{n}_{k} \\
\boldsymbol{n}_{i} ; \boldsymbol{n}_{j}
\end{array}\right) \equiv \frac{1}{\tau\left(\theta_{i j}\right)} \Omega_{d}\left[\delta\left(\widehat{\boldsymbol{n}_{j} \boldsymbol{n}_{k}}\right)-\delta\left(\widehat{\boldsymbol{n}_{i} \boldsymbol{n}_{k}}\right)\right] \equiv \gamma_{i j}^{k}
$$

This function is related to the impurity collision integral (5.8) by

$$
\begin{aligned}
\int \frac{d \boldsymbol{n}_{2}}{\Omega_{d}} \gamma\left(\begin{array}{c}
\boldsymbol{n}_{3} \\
\boldsymbol{n}_{1} ; \boldsymbol{n}_{2}
\end{array}\right) & =\left[\mathrm{St}_{\tau}\right]\left(\boldsymbol{n}_{1}, \boldsymbol{n}_{3}\right) ; \\
\int \frac{d \boldsymbol{n}_{1}}{\Omega_{d}} \gamma\left(\begin{array}{c}
\boldsymbol{n}_{3} \\
\boldsymbol{n}_{1} ; \boldsymbol{n}_{2}
\end{array}\right) & =-\left[\mathrm{St}_{\tau}\right]\left(\boldsymbol{n}_{2}, \boldsymbol{n}_{3}\right) ; \\
\int \frac{d \boldsymbol{n}_{3}}{\Omega_{d}} \gamma\left(\begin{array}{c}
\boldsymbol{n}_{3} \\
\boldsymbol{n}_{1} ; \boldsymbol{n}_{2}
\end{array}\right) & =0
\end{aligned}
$$

Denoting with $\delta g^{K}$ the first order correction to $g^{K}$, the Keldysh component of the Eilenberger equations at the linear order is:

$$
\begin{aligned}
& i\left(\tilde{\partial}_{t}+\boldsymbol{v} \cdot \tilde{\boldsymbol{\nabla}}-\widehat{\mathrm{St}}_{\tau}\right) \delta g^{K} \\
& +\left[\left(\partial_{t}+\boldsymbol{v} \cdot \boldsymbol{\nabla}-\widehat{\mathrm{St}}_{\tau}\right) K_{+}-\phi_{+} ; g^{K}\right]=\hat{Q} K_{-} \\
& +\int \frac{d \boldsymbol{n}_{2} d \boldsymbol{n}_{3}}{\Omega_{d}^{2}} \gamma\left(\begin{array}{c}
\boldsymbol{n}_{3} \\
\boldsymbol{n} ; \boldsymbol{n}_{2}
\end{array}\right)\left[K_{+}\left(\boldsymbol{n}_{3}\right) ; g^{K}\left(\boldsymbol{n}_{2}\right)-g^{K}(\boldsymbol{n})\right]
\end{aligned}
$$

with [cf. Eq. (5.11)]

$$
\begin{aligned}
& \tilde{\partial}_{t} \delta g^{K}=\left(\partial_{t_{1}}+\partial_{t_{2}}\right) \delta g^{K}+i\left[\varphi ; \delta g^{K}\right] \\
& \tilde{\nabla} \delta g^{K}=\nabla \delta g^{K}+i\left[\mathbf{A} ; \delta g^{K}\right] .
\end{aligned}
$$

Here $\hat{Q}$ is a local in space operator

$$
\hat{Q} K_{-}=\int d t_{3} \int \frac{d \boldsymbol{n}_{1}}{\Omega_{d}} Q\left(t_{1}, t_{2}, \boldsymbol{n} ; t_{3}, \boldsymbol{n}_{1} ; \boldsymbol{r}\right) K_{-}\left(t_{3}, \boldsymbol{n}_{1}, \boldsymbol{r}\right)
$$

with the kernel

$$
\begin{aligned}
& Q\left(t_{1}, t_{2}, \boldsymbol{n}_{1} ; t_{3}, \boldsymbol{n}_{2}\right)=\frac{1}{2} \int \frac{d \boldsymbol{n}_{3}}{\Omega_{d}} \gamma\left(\begin{array}{c}
\boldsymbol{n}_{2} \\
\boldsymbol{n}_{1} ; \boldsymbol{n}_{3}
\end{array}\right) \\
& \times\left[P g^{K}\left(t_{1}, t_{3}, \boldsymbol{n}_{1}\right) g^{K}\left(t_{3}, t_{2}, \boldsymbol{n}_{3}\right)+\left(\boldsymbol{n}_{1} \leftrightarrow \boldsymbol{n}_{3}\right)\right] .
\end{aligned}
$$

We suppressed the spectator argument $\boldsymbol{r}$, which is the same in each term of the equation, and the last term means that one must add terms like the ones shown but with the angular arguments of the Green's function switched. The principal value sign $P$ in Eq. (5.30) means that the divergent at $t_{1} \rightarrow t_{2}$ part of the product of the Green's functions

$$
\left.g^{K}\left(t_{1}, t_{2}, \boldsymbol{n}, \boldsymbol{r}\right)\right|_{t_{1} \rightarrow t_{2}}=-\frac{2 i}{\pi\left(t_{1}-t_{2}\right)}+\text { regular }
$$

must be excluded:

$$
\begin{aligned}
P g^{K}\left(t_{1}, t_{3}\right) & g^{K}\left(t_{3}, t_{2}\right) \\
& \equiv g^{K}\left(t_{1}, t_{3}\right) g^{K}\left(t_{3}, t_{2}\right)-4 \delta\left(t_{1}-t_{3}\right) \delta\left(t_{3}-t_{2}\right),
\end{aligned}
$$

or, equivalently,

$$
\begin{aligned}
& \operatorname{Pg}^{K}\left(t_{1}, t_{3}\right) g^{K}\left(t_{3}, t_{2}\right) \\
& \equiv \frac{1}{2} \sum_{\sigma= \pm 1} g^{K}\left(t_{1}, t_{3}+\sigma i 0\right) g^{K}\left(t_{3}+\sigma i 0, t_{2}\right)
\end{aligned}
$$

It is worth noticing that all non-equilibrium effects contribute to the regular part in Eq. (5.31) but not to the singular part - the states deep into the Fermi sea, which are not perturbed, contribute to it.

In order to solve Eq. $(5.29)$ we define a new field $\tilde{K}_{-}$ by the relation

$$
\tilde{K}_{-}(t, \boldsymbol{n}, \boldsymbol{r})=\left(i \partial_{t}\right)^{-1} \hat{M} K_{-},
$$

where the operator $\hat{M}$ will be shown to be related to certain products of the Green's functions $g^{K}$, see Eq. (5.38). The operator $\hat{M}$ is Hermitian and local in space but not in the momentum direction and time. We used again an operator notation

$$
\hat{M} K_{-} \equiv \int d t_{1} \int \frac{d \boldsymbol{n}_{1}}{\Omega_{d}} M\left(t, \boldsymbol{n} ; t_{1}, \boldsymbol{n}_{1} ; \boldsymbol{r}\right) K_{-}\left(t_{1}, \boldsymbol{n}_{1}, \boldsymbol{r}\right) .
$$

We require $K_{+}$to satisfy:

$$
\left(\partial_{t}+\boldsymbol{v} \cdot \nabla-\widehat{\mathrm{St}}_{\tau}\right) K_{+}=\phi_{+}-2 \tilde{K}_{-},
$$

whose solution is:

$$
K_{+}=\hat{\mathcal{L}}^{g} \phi_{+}-2 \hat{\mathcal{L}}^{g} \tilde{K}_{-} .
$$

The operator notation here is the same as in Eq. (5.26).

The next task is to choose the "best" form for the operator $\hat{M}$ to maximally simplify any further perturbative expansion. Writing $\delta g^{K}=\delta g_{+}^{K}+\delta g_{-}^{K}$, we obtain the following equations:

$$
\begin{aligned}
i \hat{L} \delta g_{-}^{K}= & \hat{Q} K_{-}+2\left[\tilde{K}_{-}, g^{K}\right] \\
i \hat{L} \delta g_{+}^{K}= & \int \frac{d \boldsymbol{n}_{2} d \boldsymbol{n}_{3}}{\Omega_{d}^{2}} \gamma\left(\begin{array}{c}
\boldsymbol{n}_{3} \\
\boldsymbol{n} ; \boldsymbol{n}_{2}
\end{array}\right) \\
& \times\left[K_{+}\left(\boldsymbol{n}_{3}\right),\left(g^{K}\left(\boldsymbol{n}_{2}\right)-g^{K}(\boldsymbol{n})\right)\right]
\end{aligned}
$$

where $\hat{L} \equiv\left(\tilde{\partial}_{t}+\boldsymbol{v} \cdot \tilde{\nabla}-\widehat{\mathrm{St}}_{\tau}\right)$.

We note that the right hand side of Eq. (5.36b) vanishes in equilibrium, since $g^{K}=\left\langle g^{K}\right\rangle_{\boldsymbol{n}}$. Therefore $\delta g_{+}^{K}$ also vanishes in equilibrium and cannot contribute to equilibrium properties such as the specific heat. Moreover, even in non-equilibrium $\delta g_{+}^{K}\left(t_{1}, t_{1}, \boldsymbol{n}, \boldsymbol{r}\right)=0$, because the right hand side of Eq. (5.36b) vanishes, see the remark after Eq. (5.31). It means that $\delta g_{+}^{K}$ does not contribute to the electron density or current. 
We are now ready to choose the operator $\hat{M}$. We require that $\delta g_{-}^{K}\left(t_{1}, t_{1}, \boldsymbol{n}, \boldsymbol{r}\right)=0$, i.e. $\delta g_{-}^{K}$ also does not contribute to the electron density or current. It means the the right-hand side of Eq. (5.36a) must vanish for $t_{1}=t_{2}$ for any field $K_{-}$. Imposing this requirement, we obtain

$$
\hat{M}\left(t_{1}, t_{2}, \boldsymbol{n}, \tilde{\boldsymbol{n}}, \boldsymbol{r}\right)=\frac{\pi}{4} \hat{Q}\left(t_{1}, t_{1}, \boldsymbol{n} ; t_{2}, \tilde{\boldsymbol{n}} ; \boldsymbol{r}\right) .
$$

Together with Eq. (5.30), it yields

$$
\begin{aligned}
& M\left(t_{1}, \boldsymbol{n}_{1} ; t_{2}, \boldsymbol{n}_{2} ; \boldsymbol{r}\right)=\frac{\pi}{8} \int \frac{d \boldsymbol{n}_{3}}{\Omega_{d}} \gamma\left(\begin{array}{c}
\boldsymbol{n}_{2} \\
\boldsymbol{n}_{1} ; \boldsymbol{n}_{3}
\end{array}\right) \\
& \times\left[P g^{K}\left(t_{1}, t_{2}, \boldsymbol{n}_{1}\right) g^{K}\left(t_{2}, t_{1}, \boldsymbol{n}_{3}\right)+\left(\boldsymbol{n}_{1} \leftrightarrow \boldsymbol{n}_{3}\right)\right] .
\end{aligned}
$$

Expression (5.27) for the vertex $\gamma$ enables us to establish the following properties of kernel Eq. (5.38)

$$
M\left(t_{1}, \boldsymbol{n}_{1} ; t_{2}, \boldsymbol{n}_{2}\right)=M\left(t_{1}, \boldsymbol{n}_{2} ; t_{2}, \boldsymbol{n}_{1}\right)=M\left(t_{2}, \boldsymbol{n}_{2} ; t_{1}, \boldsymbol{n}_{1}\right),
$$

i.e. the operator $\hat{M}$ is Hermitian.

It is instructive to find $\hat{M}$ in the thermal equilibrium. Using Eq. (5.14) and the fact that due to the choice (5.24) the retarded and advanced components of $\hat{g}$ are still given by Eq. (5.23), we find from Eqs. (5.28) and (5.38):

$$
\begin{aligned}
& M_{e q}\left(t_{1}, \boldsymbol{n}_{1} ; t_{2}, \boldsymbol{n}_{2} ; \boldsymbol{r}\right)=\int \frac{d \omega}{2 \pi} e^{i \omega\left(t_{2}-t_{1}\right)} \hat{M}_{e q}\left(\omega ; \boldsymbol{n}_{1}, \boldsymbol{n}_{2}\right) \\
& M_{e q}\left(\omega ; \boldsymbol{n}_{1}, \boldsymbol{n}_{2}\right)=-\omega \operatorname{coth}\left(\frac{\omega}{2 T}\right)\left[\hat{S} t_{\tau}\right]\left(\boldsymbol{n}_{1}, \boldsymbol{n}_{2}\right) .
\end{aligned}
$$

Equation (5.39) will be useful for checking the fluctuation dissipation theorem.

\section{Polarization operators and propagators}

The knowledge of the linear order corrections to the Green's function permits the calculation of the polarization operators as variational derivatives of the original Green's functions (i.e. before the gauge transformation) in the limit $t_{2} \rightarrow t_{1}$, see Eq. (5.17). At linear order the corrections to the original Green's functions are given by the relations [cf. Eq. (5.21)]:

$$
\begin{aligned}
\delta g^{K} & \rightarrow \delta g^{K}-i\left[K_{+}, g^{K}\right]-2 K_{-} \delta\left(t_{1}-t_{2}\right), \\
\delta g^{Z} & \rightarrow 2 K_{-} \delta\left(t_{1}-t_{2}\right) .
\end{aligned}
$$

By construction of the previous subsection:

$$
\lim _{t_{2} \rightarrow t_{1}} \delta g^{K}\left(t_{1}, t_{2}, \boldsymbol{n}, \boldsymbol{r}\right)=0
$$

and using Eq. (5.31):

$$
\lim _{t_{2} \rightarrow t_{1}}-i\left[K_{+}, g^{K}\right]=-\frac{2}{\pi} \partial_{t} K_{+}(t, \boldsymbol{n}, \boldsymbol{r}) .
$$

Substituting these results into Eq. (5.17) and using Eqs. (5.26), (5.32) and (5.35), we obtain:

$$
\begin{aligned}
& \Pi^{R}(1,2)=\nu\left[\delta_{12}-\partial_{t_{1}} \mathcal{L}^{g}(1,2)\right] ; \\
& \Pi^{A}(2,1)=\nu\left[\delta_{12}-\partial_{t_{2}} \overline{\mathcal{L}}^{g}(1,2)\right],
\end{aligned}
$$

and we use the notation (5.2) throughout this subsection. The result for the Keldysh component is

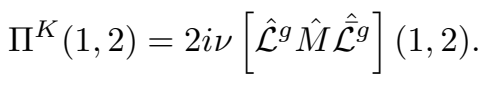

The actions of the operators $\hat{M}$ and $\hat{D}$ are defined in Eqs. (5.26) and (5.33).

It is easy to check that the fluctuation-dissipation relation between the polarization operators holds in the thermal equilibrium. As follows from Eqs. (5.25) and $(5.26)$

$$
-2 \hat{\mathcal{L}}^{g} \hat{S} t_{\tau} \hat{\overline{\mathcal{L}}}^{g}=\hat{\mathcal{L}}^{g}+\hat{\overline{\mathcal{L}}}^{g} .
$$

We perform the time Fourier transform for all the propagators and the polarization operators in thermodynamic equilibrium

$$
A(1,2)=\int \frac{d \omega}{2 \pi} e^{i \omega\left(t_{2}-t_{1}\right)} A\left(\omega ; 1^{*}, 2^{*}\right),
$$

where we use the short hand notation (5.2). Substituting Eqs. (5.39) and (5.44) into Eq. (5.43b), we obtain that in equilibrium:

$$
\Pi_{e q}^{K}\left(\omega ; 1^{*}, 2^{*}\right)=\left[\Pi^{R}\left(\omega ; 1^{*}, 2^{*}\right)-\Pi^{A}\left(\omega ; 1^{*}, 2^{*}\right)\right] \operatorname{coth} \frac{\omega}{2 T} .
$$

and with the help of Eq. (5.15), we derive the fluctuationdissipation relation

$$
D_{e q}^{K}\left(\omega ; 1^{*}, 2^{*}\right)=\left[D^{R}\left(\omega ; 1^{*}, 2^{*}\right)-D^{A}\left(\omega ; 1^{*}, 2^{*}\right)\right] \operatorname{coth} \frac{\omega}{2 T} .
$$

Given the expressions for the polarization operators obtained above, we can solve the Dyson equation (5.15) and obtain the explicit expressions for the interaction propagators. In the operator notation:

$$
\begin{aligned}
& \nu \hat{D}^{R}=-\frac{1}{1+\hat{F}-\partial_{t} \hat{F}^{\hat{\mathcal{L}}^{g}}} \hat{F} \\
& \nu \hat{D}^{A}=-\hat{F} \frac{1}{1+\hat{F}+\partial_{t} \hat{\overline{\mathcal{L}}}^{g} \hat{F}} \\
& \hat{D}^{K}=2 i \nu \hat{D}^{R} \hat{\mathcal{L}}^{g} \hat{M} \hat{\overline{\mathcal{L}}}^{g} \hat{D}^{A},
\end{aligned}
$$

where the action of the operator $\hat{F}$ on any function $a(t, \boldsymbol{n}, \boldsymbol{r})$ is defined by

$$
\begin{gathered}
{[\hat{F} a](t ; \boldsymbol{n}, \boldsymbol{r}) \equiv \int \frac{d \boldsymbol{n}_{1}}{\Omega_{d}}\left[F^{\rho}\left(\widehat{\boldsymbol{n n}} \boldsymbol{n}_{1}\right) a\left(t, \boldsymbol{n}_{1}, \boldsymbol{r}\right)\right.} \\
\left.+\int d \boldsymbol{r}_{1} \nu V\left(\boldsymbol{r}-\boldsymbol{r}_{1}\right) a\left(t, \boldsymbol{n}_{1}, \boldsymbol{r}_{1}\right)\right]
\end{gathered}
$$

see also the text after Eq. (5.15).

To find the propagators for the fields $K_{ \pm}$given in 
Eqs. $\left(5.26^{\prime}\right)$ and (5.35), defined as:

$$
\begin{aligned}
\left\langle\left\langle K_{+}(1) K_{+}(2)\right\rangle\right\rangle & =\frac{i}{2} \mathcal{K}^{K}(1,2), \\
\left\langle\left\langle K_{+}(1) K_{-}(2)\right\rangle\right\rangle & =\frac{i}{2} \mathcal{K}^{R}(1,2), \\
\left\langle\left\langle K_{-}(1) K_{+}(2)\right\rangle\right\rangle & =\frac{i}{2} \mathcal{K}^{A}(1,2), \\
\left\langle\left\langle K_{-}(1) K_{-}(2)\right\rangle\right\rangle & =0
\end{aligned}
$$

we use Eqs. (5.1) and (5.48) and obtain for the retarded and advanced propagators

$$
\hat{\mathcal{K}}^{R}=\hat{\mathcal{L}}^{g} \hat{D}^{R} \hat{\mathcal{L}}^{g} ; \quad \hat{\mathcal{K}}^{A}=\hat{\overline{\mathcal{L}}}^{g} \hat{D}^{A} \hat{\mathcal{\mathcal { L }}^{g}},
$$

whereas the result for the Keldysh propagator is

$$
\begin{aligned}
\hat{\mathcal{K}}^{K}= & -\hat{\mathcal{L}}^{g} \hat{D}^{K} \hat{\hat{\mathcal{L}}^{g}} \\
& +2 i\left[\hat{\mathcal{L}}^{g}\left(\partial_{t}\right)^{-1} \hat{M} \hat{\hat{\mathcal{L}}^{g}} \hat{D}^{A} \hat{\overline{\mathcal{L}^{g}}}-\hat{\mathcal{L}}^{g} \hat{D}^{R} \hat{\mathcal{L}}^{g} \hat{M}\left(\partial_{t}\right)^{-1} \hat{\hat{\mathcal{L}}^{g}}\right] .
\end{aligned}
$$

The fluctuation-dissipation relation between the $D$ propagators in Eq. (5.47), the equilibrium form for $\hat{M}$ in Eq. (5.39) and the identity (5.44) enable us to verify the fluctuation-dissipation relation for the $\mathcal{K}$ propagators:

$$
\mathcal{K}_{e q}^{K}\left(\omega ; 1^{*}, 2^{*}\right)=\left[\mathcal{K}^{R}\left(\omega ; 1^{*}, 2^{*}\right)-\mathcal{K}^{A}\left(\omega ; 1^{*}, 2^{*}\right)\right] \operatorname{coth} \frac{\omega}{2 T} .
$$

\section{Additional bosonic fields}

Equation (5.51b) together with Eqs. (5.51a) and (5.38) permits to express the Keldysh propagator $\mathcal{K}^{K}$ in terms of the electron distribution function. This relation, however, would be nonlocal on the spatial scale much larger than the temperature length

$$
L_{T} \simeq \min \left[\frac{\hbar v_{F}}{T}, v_{F} \sqrt{\frac{\hbar \tau}{T}}\right],
$$

recall the discussion of Sec. II B. Indeed the collision integral and all the physical quantities will be given by integrals of the type

$$
\mathcal{I}^{\alpha}=\int d \omega f(\omega) \mathcal{K}^{\alpha}(\omega), \quad \alpha=R, A, K,
$$

where the function $f(\omega)$ depends on its argument on the characteristic scale of $T$. A retarded function is an analytic function of $\omega$ at $\operatorname{Im} \omega>0$, which means that for $\alpha=R$ the integral will be determined only by the singularities of $f(\omega)$, i.e. $\mathcal{I}^{R} \simeq \mathcal{K}^{R}(\omega=i T)$. This immediately restricts the spatial scales to $L_{T}$. The same argument applies to the advanced case, because of analyticity at $\operatorname{Im} \omega<0$. The function $\mathcal{K}^{K}(\omega)$, however, is not analytic. Moreover, according to Eq. (5.51b) it contains overlapping singularities of the retarded and advanced propagators. It means that the characteristic frequencies entering $\mathcal{I}^{K}$ are determined by the poles of the propagator rather than by the width of the function $f$, i.e. the spatial scale may by far exceed $L_{T}$ and any expression of the type $\mathcal{I}^{K}$ is thus non-local.

To overcome this difficulty, the standard parametrization of the Keldysh function $D^{K}=D^{R} \circ N-N \circ D^{A}$ is usually introduced and the kinetic equation for the distribution function $N$ is then derived. All the non-locality in the problem is then contained in the partial solution of the kinetic equation, compare with Eq. (2.14), whereas the kinetic equation itself is local.

In what follows, we adopt this program in a slightly modified form. We introduce a new retarded propagator $\mathcal{L}^{\rho}(1,2)$, compare with Eq. (5.25)

$$
\begin{aligned}
& {\left[i \hat{H}_{\mathrm{e}-\mathrm{h}}\left(i \partial_{t_{1}},-i \boldsymbol{\nabla}_{1}\right)-\widehat{\mathrm{St}}_{\tau}\right] \mathcal{L}^{\rho}=\delta_{12},} \\
& \hat{H}_{\mathrm{e}-\mathrm{h}}(\omega, \boldsymbol{q})=\boldsymbol{v} \cdot \boldsymbol{q}-\frac{\omega}{1+\hat{F}}
\end{aligned}
$$

and its advanced counterpart $\overline{\mathcal{L}}^{\rho}(1,2)=\mathcal{L}^{\rho}(2,1)$. The multiplications in Eq. (5.54) are to be understood in the operator sense and the action of the operator $\hat{F}$ on a function $a(t, \boldsymbol{n}, \boldsymbol{r})$ is defined by Eq. (5.49).

For $\hat{F}=0, \mathcal{L}^{\rho}\left(\overline{\mathcal{L}}^{\rho}\right)$ reduces to the usual diffuson $\mathcal{L}^{g}$ $\left(\overline{\mathcal{L}}^{g}\right)$. Physically, $\mathcal{L}^{\rho}$ describes the spectrum of the propagating electron-hole pair and the operator in the left hand side of Eq. (5.54) corresponds to the kinetic equation for the collective mode in the Fermi liquid theory ${ }^{26}$. The operator $\hat{H}_{\mathrm{e}-\mathrm{h}}(\omega, \boldsymbol{q})$ can be interpreted as a "Hamiltonian" [see also App. C] of the interacting electron-hole pair.

In terms of $\mathcal{L}^{\rho}$ and $\mathcal{L}^{g}$, Eqs. (5.51) acquire the form

$$
\begin{gathered}
\nu \hat{\mathcal{K}}^{R}=\left(\partial_{t}\right)^{-1}\left[\hat{\mathcal{L}}^{g}-\hat{\mathcal{L}}^{\rho}\right] \\
\nu \hat{\mathcal{K}}^{A}=-\left[\hat{\mathcal{\mathcal { L }}^{g}}-\hat{\overline{\mathcal{L}}}^{\rho}\right]\left(\partial_{t}\right)^{-1} \\
\nu \hat{\mathcal{K}}^{K}=-2 i\left(\partial_{t}\right)^{-1}\left[\hat{\mathcal{L}}^{g} \hat{M} \hat{\overline{\mathcal{L}}}^{g}-\hat{\mathcal{L}}^{\rho} \hat{\bar{M}} \hat{\overline{\mathcal{L}}}^{\rho}\right]\left(\partial_{t}\right)^{-1} .
\end{gathered}
$$

Let us introduce two bosonic "distribution functions" (the density matrices to be more precise), $\hat{\mathcal{N}}^{g}$ and $\hat{\mathcal{N}}^{\rho}$, that satisfy the following equations:

$$
\begin{aligned}
& \left(\hat{\mathcal{L}}^{g}\right)^{-1} \hat{\mathcal{N}}^{g}+\hat{\mathcal{N}}^{g}\left(\hat{\overline{\mathcal{L}}}^{g}\right)^{-1}=2 \hat{M}, \\
& \left(\hat{\mathcal{L}}^{\rho}\right)^{-1} \hat{\mathcal{N}}^{\rho}+\hat{\mathcal{N}}^{\rho}\left(\hat{\overline{\mathcal{L}}}^{\rho}\right)^{-1}=2 \hat{M} .
\end{aligned}
$$

The operator $\hat{M}$ is defined in Eqs. (5.33) and (5.38) and, in a more explicit notation, the action of the operators $\hat{\mathcal{N}}^{\rho, g}$ on any function $a(t, \boldsymbol{n}, \boldsymbol{r})$ is to be understood as

$$
\left[\hat{\mathcal{N}}^{\rho, g} a\right](1)=\int d 2 \mathcal{N}^{\rho, g}(1,2) a(2),
$$

where the shorthand notation (5.2) is used. Note that from the above equations it follows that the bosonic functions $\mathcal{N}^{\rho, g}$ are symmetric

$$
\mathcal{N}^{\rho, g}(1,2)=\mathcal{N}^{\rho, g}(2,1) .
$$


Equations (5.56) enable us to rewrite Eq. (5.55b) as

$$
\begin{aligned}
\nu \hat{\mathcal{K}}^{K}= & -i\left(\partial_{t}\right)^{-1}\left[\hat{\mathcal{L}}^{g} \hat{\mathcal{N}}^{g}+\hat{\mathcal{N}}^{g} \hat{\mathcal{L}}^{g}\right]\left(\partial_{t}\right)^{-1} \\
& +i\left(\partial_{t}\right)^{-1}\left[\hat{\mathcal{L}}^{\rho} \hat{\mathcal{N}}^{\rho}+\hat{\mathcal{N}}^{\rho} \hat{\overline{\mathcal{L}}}^{\rho}\right]\left(\partial_{t}\right)^{-1} .
\end{aligned}
$$

This expression is local in the above discussed sense and will be used in the construction of the conserved energy current. Obtaining the local expression, however, required the introduction of two additional bosonic distribution functions: $\mathcal{N}^{\rho}$, describing the interacting electronhole pairs and the ghost field distribution $\mathcal{N}^{g}$, subtracting the contribution of the electron-hole pairs in the absence of interactions.

Closing this subsection, let us rewrite Eqs. (5.56) in a form resembling the kinetic equation of Sec. II. We substitute Eqs. (5.25) and (5.54) in Eqs. (5.56) and obtain

$$
\begin{aligned}
{\left[\partial_{t}+\boldsymbol{v} \cdot \boldsymbol{\nabla} ; \hat{\mathcal{N}}^{g}\right] } & =\widehat{\mathrm{St}}^{b}\left\{\mathcal{N}^{g}, g^{K}\right\} \\
{\left[i \hat{H}_{\mathrm{e}-\mathrm{h}}\left(i \partial_{t_{1}},-i \boldsymbol{\nabla}_{1}\right) ; \hat{\mathcal{N}}^{\rho}\right] } & =\widehat{\mathrm{St}}^{b}\left\{\mathcal{N}^{\rho}, g^{K}\right\}
\end{aligned}
$$

where the collision integrals are

$$
\widehat{\mathrm{St}}^{b}\left\{\mathcal{N}^{\alpha}, g^{K}\right\} \equiv 2\left\{\widehat{\mathrm{St}}_{\tau} ; \hat{\mathcal{N}}^{\alpha}\right\}+2 \hat{M}
$$

for $\alpha=g, \rho$. They depend on $g^{K}$ via $\hat{M}$ and we used the notation

$$
\{\hat{A} ; \hat{B}\} \equiv \frac{1}{2}(\hat{A} \hat{B}+\hat{B} \hat{A}) ; \quad[\hat{A} ; \hat{B}] \equiv \hat{A} \hat{B}-\hat{B} \hat{A} .
$$

We perform the time and space Wigner transforms of Eqs. (5.59) to introduce the bosonic distribution functions $N^{g, \rho}$

$$
\begin{aligned}
& \hat{\mathcal{N}}^{g, \rho}(1,2)=\int \frac{d \omega}{2 \pi} e^{-i \omega\left(t_{1}-t_{2}\right)} \int \frac{d^{d} q}{(2 \pi)^{d}} e^{i \boldsymbol{q} \cdot\left(\boldsymbol{r}_{1}-\boldsymbol{r}_{2}\right)} \\
& \times \omega\left[2 N^{g, \rho}\left(\omega, \boldsymbol{q} ; \boldsymbol{n}_{1}, \boldsymbol{n}_{2} ; t, \boldsymbol{r}\right)+\Omega_{d} \delta\left(\widehat{\boldsymbol{n}_{1} \boldsymbol{n}_{2}}\right)\right],
\end{aligned}
$$

where $t=\frac{t_{1}+t_{2}}{2}, \boldsymbol{r}=\frac{\boldsymbol{r}_{1}+\boldsymbol{r}_{2}}{2}$. The symmetry relation (5.57) translates into the condition

$$
\begin{aligned}
& N^{g, \rho}\left(\omega, \boldsymbol{q} ; \boldsymbol{n}_{1}, \boldsymbol{n}_{2}\right) \\
& \quad=-\left[N^{g, \rho}\left(-\omega,-\boldsymbol{q} ; \boldsymbol{n}_{2}, \boldsymbol{n}_{1}\right)+\Omega_{d} \delta\left(\widehat{\boldsymbol{n}_{1} \boldsymbol{n}_{2}}\right)\right] .
\end{aligned}
$$

The physical meaning of this relation is boson statistics: at $\omega>0, N^{g, \rho}$ corresponds to the occupation numbers entering the probability of the absorption of the bosons whereas the $\omega<0$ part describes the boson emission.

The fermionic distribution function $f$ is obtained in two steps: (a) we introduce the gauge invariant Green's function $g$ (see also the next subsection) and (b) we per- form the time Wigner transform:

$$
\begin{aligned}
g^{K}\left(t_{1}, t_{2}, \boldsymbol{n}, \boldsymbol{r}\right) & \equiv \exp \left(-i \int_{t_{2}}^{t_{1}} d \tilde{t} \varphi(\tilde{t}, \boldsymbol{r})\right) g\left(t_{1}, t_{2}, \boldsymbol{n}, \boldsymbol{r}\right) \\
g\left(t_{1}, t_{2}, \boldsymbol{n}, \boldsymbol{r}\right) & =2 \int \frac{d \epsilon}{2 \pi} e^{-i \epsilon\left(t_{1}-t_{2}\right)}[1-2 f(\epsilon, \boldsymbol{n} ; t, \boldsymbol{r})] .
\end{aligned}
$$

Performing such Wigner transforms on Eqs. (5.56) and (5.38), we find

$$
\begin{gathered}
\omega\left[\partial_{t} \hat{N}^{g}+\left\{\boldsymbol{v} ; \boldsymbol{\nabla} \hat{N}^{g}\right\}+i\left[\boldsymbol{v} \cdot \boldsymbol{q} ; \hat{N}^{g}\right]\right]=\widehat{\mathrm{St}}^{b}\left\{N^{g}, f\right\} \\
\omega\left[\left\{\frac{1}{1+\hat{F}} ; \partial_{t} \hat{N}^{\rho}\right\}+\left\{\hat{\boldsymbol{s}}(\omega, \boldsymbol{q}) ; \boldsymbol{\nabla} \hat{N}^{\rho}\right\}\right. \\
\left.+i\left[\hat{H}_{\mathrm{e}-\mathrm{h}}(\omega, \boldsymbol{q}), \hat{N}^{\rho}\right]\right]=\widehat{\mathrm{St}}^{b}\left\{N^{\rho}, f\right\}
\end{gathered}
$$

where the collective mode velocity operator is

$$
\hat{\boldsymbol{s}}(\omega, \boldsymbol{q})=\frac{\partial \hat{H}_{\mathrm{e}-\mathrm{h}}(\omega, \boldsymbol{q})}{\partial \boldsymbol{q}}=\boldsymbol{v}+\omega \frac{\partial}{\partial \boldsymbol{q}}\left(\frac{\hat{F}}{1+\hat{F}}\right) .
$$

In the left hand side of equation (5.64b) we limited ourselves to the leading Poisson brackets [the equation becomes exact for the short range interaction, since $\partial_{\boldsymbol{q}} \hat{F}=$ 0 , and in the unitary limit $\hat{F} \rightarrow \infty]$. However no Poisson brackets arise in the right hand sides of Eqs. (5.64) - this is a consequence of the locality of the kinetic equations.

The right hand sides of Eqs. (5.64) describe the decay of the electron-hole pair into the electron and the hole moving into different directions. To write down the expression for this collision term, it is convenient to introduce the following object:

$$
\begin{aligned}
& \Upsilon_{i j ; k l}^{g, \rho}(\epsilon, \omega, \boldsymbol{q} ; t, \boldsymbol{r}) \equiv N^{g, \rho}\left(\omega, \boldsymbol{q} ; \boldsymbol{n}_{i}, \boldsymbol{n}_{j} ; t, \boldsymbol{r}\right) \\
& \quad \times\left\{f\left(\epsilon, \boldsymbol{n}_{k} ; t, \boldsymbol{r}\right)-f\left(\epsilon-\omega, \boldsymbol{n}_{k} ; t, \boldsymbol{r}\right)\right\} \\
& \quad+\Omega_{d} \delta\left(\widehat{\boldsymbol{n}_{i} \boldsymbol{n}_{j}}\right)\left\{f\left(\epsilon, \boldsymbol{n}_{l} ; t, \boldsymbol{r}\right)\left[1-f\left(\epsilon-\omega, \boldsymbol{n}_{k} ; t, \boldsymbol{r}\right)\right]\right\} .
\end{aligned}
$$

It is easy to see that $\Upsilon^{g, \rho}=0$ in the thermal equilibrium Eq. (3.1).

In terms of this object and the vertex (5.27) we have

$$
\begin{aligned}
\widehat{\mathrm{St}^{b}} & \left\{N^{g, \rho}, f\right\}\left(\omega, \boldsymbol{q} ; \boldsymbol{n}_{1}, \boldsymbol{n}_{2} ; t, \boldsymbol{r}\right)=-\int d \epsilon \int \frac{d \boldsymbol{n}_{3} d \boldsymbol{n}_{4}}{\Omega_{d}^{2}} \\
& \times\left\{\gamma_{14}^{3} \Upsilon_{32 ; 41}^{g, \rho}(\epsilon, \omega)+\gamma_{34}^{2} \Upsilon_{13 ; 14}^{g, \rho}(\epsilon, \omega)\right\},
\end{aligned}
$$

where we suppressed the spectator arguments $t, \boldsymbol{r}$ and $\boldsymbol{q}$ in the right-hand side of the equation. Deriving Eq. (5.67) we used Eqs. (5.28), (5.38') and the property

$$
\int d \epsilon[f(\epsilon)-f(\epsilon-\omega)]=-\omega
$$


To understand the physical meaning of the processes described by the collision integral (5.67), we use the explicit form of the vertex $\gamma$ [Eq. (5.27)] for the isotropic impurity scattering $\tau\left(\theta_{12}\right)=\tau$. Then, the collision integrals can be decomposed into the sum of two contributions

$$
\widehat{\mathrm{St}}^{b}\left\{N^{g, \rho}, f\right\}=\widehat{\mathrm{St}}_{c l}^{b}\left\{N^{g, \rho}, f\right\}+\widehat{\mathrm{St}}_{q}^{b}\left\{N^{g, \rho}, f\right\}
$$

The first term in the right-hand side can be obtained from a simple counting of the probabilities of the processes depicted on Fig. 2(a-b)

$$
\begin{aligned}
\widehat{\mathrm{St}}_{c l}^{b} & \left\{N^{g, \rho}, f\right\}\left(\omega ; \boldsymbol{n}_{1}, \boldsymbol{n}_{2}\right) \\
& =\frac{1}{\tau} \int d \epsilon \int \frac{d \boldsymbol{n}_{3}}{\Omega_{d}}\left\{\Upsilon_{12 ; 32}^{g, \rho}(\epsilon, \omega)+\Upsilon_{12 ; 13}^{g, \rho}(\epsilon, \omega, \boldsymbol{q})\right\} .
\end{aligned}
$$

The second term in the right-hand side originates from the interference of two scattering processes, see Fig. 2(c). That is why it produces contributions to $\hat{N}$ which are not diagonal in the momentum directions:

$$
\begin{aligned}
\widehat{\mathrm{St}}_{q}^{b} & \left\{N^{g, \rho}, f\right\}\left(\omega ; \boldsymbol{n}_{1}, \boldsymbol{n}_{2}\right) \\
& =-\frac{1}{\tau} \int d \epsilon \int \frac{d \boldsymbol{n}_{3}}{\Omega_{d}}\left\{\Upsilon_{13 ; 12}^{g, \rho}(\epsilon, \omega)+\Upsilon_{32 ; 21}^{g, \rho}(\epsilon, \omega)\right\}
\end{aligned}
$$

\section{E. The collision integral for electrons}

With the bosonic propagators $\mathcal{K}$ at hand, we can proceed with the calculation and include the second order in the fluctuating fields $K_{ \pm}$contributions to the collision term of the Eilenberger equation (5.22). With the fluctuating fields $K_{ \pm}$given by Eqs. $\left(5.26^{\prime}\right)$ and (5.35), Eilenberger equation becomes:

$$
\begin{aligned}
& {\left[\tilde{\partial}_{t}+\boldsymbol{v} \cdot \tilde{\nabla}\right] \hat{g}} \\
& =\left[\hat{g} \circ \frac{1}{2} \widehat{\mathrm{St}}_{\tau}^{\phi} \hat{g}-i\left(\widehat{\mathrm{St}}_{\tau} K_{+}-2 \tilde{K}_{-}\right) \hat{\mathbb{1}}_{K}+i \widehat{\mathrm{St}}_{\tau} K_{-} \hat{\sigma}_{K}^{x}\right],
\end{aligned}
$$

where we use the notation (5.11) for the derivatives, $\widehat{\mathrm{St}}_{\tau}^{\phi}$ was defined after Eq. (5.22) and $\tilde{K}_{-}$in Eq. (5.32), and $\hat{\sigma}_{K}^{x}$ is the Pauli matrix.

We expand the right hand side of Eq. (5.68) up to the second order in $\hat{K}$, see Fig. 3a; then we average it to obtain Fig. 3b. The resulting second order contributions can have two different origins: (1) they may arise from the expansion of the exponentials truncated at the second order, term $\widehat{\mathrm{St}}_{1}$ on Fig. 3c, or (2) they are obtained as products between the linear correction $\delta g^{K}$ of Eqs. (5.36a) and the first order expansion of the exponentials, term $\widehat{\mathrm{St}}_{2}$ on Fig. 3c.

The Eilenberger equation for the averaged Green function takes the form

$$
\left[\tilde{\partial}_{t}+\boldsymbol{v} \cdot \tilde{\nabla}\right] \hat{g}=\hat{\widehat{\operatorname{St}}}\left\{\hat{g}, N^{\rho}, N^{g}\right\}
$$

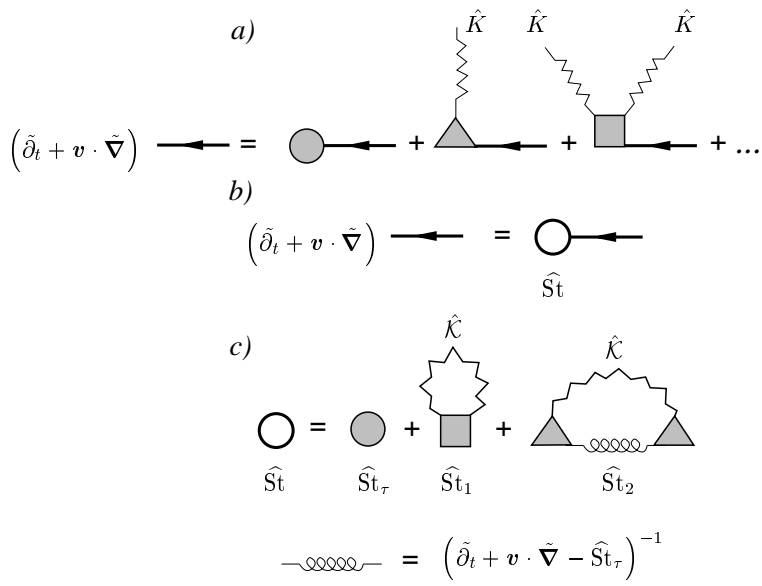

FIG. 3: Schematic representation of the averaging over the fluctuation fields $\hat{K}$. a) Expansion of the Eilenberger equation (5.68) before the averaging; b) The equation for the Green function averaged over $\hat{K}$, see Eq. (5.69); c) The contribtutions to the total collision integral in the first loop approximation.

where $\hat{\widehat{S t}}$ contains both zero and second order contributions. We find [see Appendix D for details on the cancellation of the second order corrections in the R,A,Z sectors]:

$$
\hat{\widehat{\mathrm{St}}}\left\{g_{0}^{R}, g_{0}^{A}, g^{K}\right\}=\left(\begin{array}{cc}
0 & \widehat{\mathrm{St}} \\
0 & \left.g^{K}, N^{\rho}, N^{g}\right\} \\
0
\end{array}\right),
$$

where $g_{0}^{R}=-g_{0}^{A}=\delta\left(t_{1}-t_{2}\right)$. It means that the matrix Green's function of the form (5.23) is still the solution of the Eilenberger equation - the main gain of the gauge transformation (5.21) - provided that the kinetic equation for the Keldysh component is satisfied: accordingly, we concentrate on this component only.

Performing the gauge transformation (5.63a) on the Keldysh component of the Eilenberger equation, we arrive at the explicitly gauge-invariant form of the kinetic equation:

$\left[\partial_{t_{1}}+\partial_{t_{2}}+\boldsymbol{v} \cdot \boldsymbol{\nabla}+i \boldsymbol{v} \cdot \int_{t_{2}}^{t_{1}} d \tilde{t} e \boldsymbol{E}(\tilde{t}, \boldsymbol{r})\right] g=\widehat{\mathrm{St}}\left\{g, N^{\rho}, N^{g}\right\}$

In the collision integral $\widehat{\mathrm{St}}\left\{g, N^{\rho}, N^{g}\right\}$, we indicate with $\widehat{\operatorname{St}}_{1}\left\{g, N^{\rho}, N^{g}\right\}$ the contributions of the type (1) and with $\widehat{\operatorname{St}}_{2}\left\{g, N^{\rho}, N^{g}\right\}$ those of type (2) [this separation has no particular physical meaning - it is just a matter of practicality in the calculations; we will return on the physical aspects when analyzing the conservation laws in the next subsection]:

$\widehat{\mathrm{St}}\left\{g, N^{\rho}, N^{g}\right\}=\widehat{\mathrm{St}}_{\tau} g-4 \widehat{\mathrm{St}}_{1}\left\{g, N^{\rho}, N^{g}\right\}-4 \widehat{\mathrm{St}}_{2}\left\{g, N^{\rho}, N^{g}\right\}$.

where $\widehat{S t}_{\tau}$ is defined in Eq. (5.8). The numerical factors in front of the last two terms are introduced to facilitate the transformation to the canonical form of the kinetic equation in further sections. 
The expression for $\widehat{S t}_{1}$ written in terms of the $\mathcal{K}$ propagators (5.50) and the $\gamma$-vertex (5.27) is

$$
\begin{aligned}
& {\left[\widehat{\mathrm{St}}_{1}\right]\left(t_{1}, t_{2} ; \boldsymbol{n}_{1}\right)=-\frac{i}{16} \int \frac{d \boldsymbol{n}_{2} d \boldsymbol{n}_{3}}{\Omega_{d}^{2}} \gamma_{12}^{3}} \\
& \times\left\{g\left(t_{1}, t_{2}, \boldsymbol{n}_{2}\right)\left[\tilde{\mathcal{K}}_{32}^{K}\left(t_{1}, t_{2}\right)-\tilde{\mathcal{K}}_{31}^{K}\left(t_{1}, t_{2}\right)\right]\right. \\
& +\int d t_{3} g\left(t_{1}, t_{3}, \boldsymbol{n}_{1}\right) g\left(t_{3}, t_{2}, \boldsymbol{n}_{2}\right)\left[\mathcal{K}_{32}^{A}\left(t_{3}, t_{2}\right)-\mathcal{K}_{31}^{A}\left(t_{3}, t_{2}\right)\right] \\
& \left.-g\left(t_{1}, t_{3}, \boldsymbol{n}_{2}\right) g\left(t_{3}, t_{2}, \boldsymbol{n}_{1}\right)\left[\mathcal{K}_{23}^{R}\left(t_{1}, t_{3}\right)-\mathcal{K}_{13}^{R}\left(t_{1}, t_{3}\right)\right]\right\} .
\end{aligned}
$$

We introduced the short hand notations

$$
\mathcal{K}_{i j}\left(t_{1}, t_{2}\right) \equiv \mathcal{K}\left(t_{1}, \boldsymbol{n}_{i} ; t_{2}, \boldsymbol{n}_{j}\right),
$$

and

$$
\tilde{\mathcal{K}}^{K}\left(t_{1}, t_{2}\right) \equiv 2 \mathcal{K}^{K}\left(t_{1}, t_{2}\right)-\mathcal{K}^{K}\left(t_{1}, t_{1}\right)-\mathcal{K}^{K}\left(t_{2}, t_{2}\right)
$$

We omitted the variable $\boldsymbol{r}$, which always appears in the distribution function as $g\left(t_{1}, t_{2}, \boldsymbol{n}, \boldsymbol{r}\right)$ and in the propagators as $\mathcal{K}\left(t_{1}, \boldsymbol{n}_{1}, \boldsymbol{r} ; t_{2}, \boldsymbol{n}_{2}, \boldsymbol{r}\right)$. The dependence on the electron distribution function $g$ is explicit, whereas the dependence on the bosonic distribution functions is hidden into the propagators - see Eq. (5.58). For reasons which will become clear in the next subsection, we split Eq. (5.73) into two parts

$$
\begin{aligned}
& \widehat{\mathrm{St}}_{1}=\widehat{\mathrm{St}}_{1}^{\mathrm{in}}+\widehat{\mathrm{St}}_{1}^{\mathrm{el}} ; \\
& {\left[\widehat{\mathrm{St}}_{1}^{\mathrm{in}}\right]\left(t_{1}, t_{2} ; \boldsymbol{n}_{1}\right)=-\frac{i}{32} \int \frac{d \boldsymbol{n}_{2} d \boldsymbol{n}_{3}}{\Omega_{d}^{2}} \gamma_{12}^{3}} \\
& \times\left\{2 g\left(t_{1}, t_{2}, \boldsymbol{n}_{2}\right)\left[\tilde{\mathcal{K}}_{32}^{K}\left(t_{1}, t_{2}\right)-\tilde{\mathcal{K}}_{31}^{K}\left(t_{1}, t_{2}\right)\right]\right. \\
& +\int d t_{3}\left[g\left(t_{1}, t_{3}, \boldsymbol{n}_{1}\right) g\left(t_{3}, t_{2}, \boldsymbol{n}_{2}\right)+g\left(t_{1}, t_{3}, \boldsymbol{n}_{2}\right) g\left(t_{3}, t_{2}, \boldsymbol{n}_{1}\right)\right] \\
& \left.\times\left[\mathcal{K}_{32}^{A}\left(t_{3}, t_{2}\right)-\mathcal{K}_{23}^{R}\left(t_{1}, t_{3}\right)-\mathcal{K}_{31}^{A}\left(t_{3}, t_{2}\right)+\mathcal{K}_{13}^{R}\left(t_{1}, t_{3}\right)\right]\right\} . \\
& {\left[\widehat{\mathrm{St}}_{1}^{\mathrm{el}}\right]\left(t_{1}, t_{2} ; \boldsymbol{n}_{1}\right)=-\frac{i}{32} \int d t_{3} \int \frac{d \boldsymbol{n}_{2} d \boldsymbol{n}_{3}}{\Omega_{d}^{2}} \gamma_{12}^{3}} \\
& \times\left\{\left[g\left(t_{1}, t_{3}, \boldsymbol{n}_{1}\right) g\left(t_{3}, t_{2}, \boldsymbol{n}_{2}\right)-g\left(t_{1}, t_{3}, \boldsymbol{n}_{2}\right) g\left(t_{3}, t_{2}, \boldsymbol{n}_{1}\right)\right]\right. \\
& \left.\times\left[\mathcal{K}_{32}^{A}\left(t_{3}, t_{2}\right)+\mathcal{K}_{23}^{R}\left(t_{1}, t_{3}\right)-\mathcal{K}_{31}^{A}\left(t_{3}, t_{2}\right)-\mathcal{K}_{13}^{R}\left(t_{1}, t_{3}\right)\right]\right\} .
\end{aligned}
$$

As for $\widehat{\mathrm{St}}_{2}$, it is convenient to separate it in two parts, depending on which field, $K_{+}$or $K_{-}$, we retain in the expansion:

$$
\widehat{\mathrm{St}}_{2}=\left\langle\left\langle\widehat{\mathrm{St}}_{+}\right\rangle\right\rangle+\left\langle\left\langle\widehat{\mathrm{St}}_{-}\right\rangle\right\rangle
$$

where $\langle\langle\ldots\rangle\rangle$ stands for the averaging over the fluctuating fields $K_{ \pm}$with the propagators (5.50) and

$$
\begin{aligned}
& \widehat{\mathrm{St}_{-}}\left(t_{1}, t_{2} ; \boldsymbol{n}, \boldsymbol{r}\right)=\frac{i}{4} \int d t_{3} \int \frac{d \boldsymbol{n}_{1}}{\Omega_{d}} K_{-}\left(t_{3}, \boldsymbol{n}_{1}, \boldsymbol{r}\right) \\
& \times {\left[\delta Q\left(t_{1}, t_{2}, \boldsymbol{n} ; t_{3}, \boldsymbol{n}_{1} ; \boldsymbol{r}\right)\right.} \\
&-\left.\frac{i \pi}{2} \delta g_{+}\left(t_{1}, t_{2} ; \boldsymbol{n}, \boldsymbol{r}\right) \int_{t_{2}}^{t_{1}} d t_{5} Q\left(t_{5}, t_{5}, \boldsymbol{n} ; t_{3}, \boldsymbol{n}_{1} ; \boldsymbol{r}\right)\right] . \\
& \widehat{\mathrm{St}}_{+}\left(t_{1}, t_{2} \boldsymbol{n}\right)=\frac{i}{4} \int \frac{d \boldsymbol{n}_{2} d \boldsymbol{n}_{3}}{\Omega_{d}^{2}} \gamma\left(\begin{array}{c}
\boldsymbol{n}_{3} \\
\boldsymbol{n} ; \boldsymbol{n}_{2}
\end{array}\right) \\
& \quad \times\left[K_{+}\left(\boldsymbol{n}_{3}\right) ;\left(\delta g\left(\boldsymbol{n}_{2}\right)-\delta g(\boldsymbol{n})\right)\right]\left(t_{1}, t_{2}\right) .
\end{aligned}
$$

The commutator was defined in Eq. (5.10) and the kernel $\delta Q$ is the first variation of the operator (5.30) with respect to the Keldysh component of the electron Green's function

$$
\begin{aligned}
& \delta Q\left(t_{1}, t_{2}, \boldsymbol{n} ; t_{3}, \boldsymbol{n}_{1} ; \boldsymbol{r}\right)=\frac{1}{2} \int \frac{d \boldsymbol{n}_{2}}{\Omega_{d}} \gamma\left(\begin{array}{c}
\boldsymbol{n}_{1} \\
\boldsymbol{n} ; \boldsymbol{n}_{2}
\end{array}\right) \\
& \times\left\{\left[\delta g_{+}\left(t_{1}, t_{3}, \boldsymbol{n}, \boldsymbol{r}\right) g\left(t_{3}, t_{2}, \boldsymbol{n}_{2}, \boldsymbol{r}\right)\right.\right. \\
& \left.\left.\quad+g\left(t_{1}, t_{3}, \boldsymbol{n}, \boldsymbol{r}\right) \delta g_{+}\left(t_{3}, t_{2}, \boldsymbol{n}_{2}, \boldsymbol{r}\right)\right]+\left(\boldsymbol{n}_{2} \leftrightarrow \boldsymbol{n}\right)\right\} .
\end{aligned}
$$

Finally, the functions $\delta g_{ \pm}$and $\delta g=\delta g_{+}+\delta g_{-}$are obtained by solving Eqs. (5.36a) and (5.36b) [after the transformation (5.63a)]; with the help of Eq. (5.37):

$$
\begin{gathered}
\delta g_{+}\left(t_{1}, t_{2} ; \boldsymbol{n}, \boldsymbol{r}\right)=-i \int d 3 \int \frac{d \boldsymbol{n}_{2} d \boldsymbol{n}_{4}}{\Omega_{d}^{2}} \gamma_{32}^{4} \\
\mathcal{L}^{g}\left(t_{1}, \boldsymbol{n}, \boldsymbol{r} ; 3\right)\left[K_{+}\left(t_{3}, \boldsymbol{n}_{4}, \boldsymbol{r}_{3}\right)-K_{+}\left(t_{3}-t_{12}, \boldsymbol{n}_{4}, \boldsymbol{r}_{3}\right)\right] \\
\times\left[g^{K}\left(t_{3}, t_{3}-t_{12} ; \boldsymbol{n}_{2}, \boldsymbol{r}_{3}\right)-g^{K}\left(t_{3}, t_{3}-t_{12} ; \boldsymbol{n}_{3}, \boldsymbol{r}_{3}\right)\right] \\
\delta g_{-}\left(t_{1}, t_{2} ; \boldsymbol{n}, \boldsymbol{r}\right)=-i \int d 3 \int d t_{4} \int \frac{d \boldsymbol{n}_{4}}{\Omega_{d}} \\
\mathcal{L}^{g}\left(t_{1}, \boldsymbol{n}, \boldsymbol{r} ; 3\right) K_{-}\left(t_{4}, \boldsymbol{n}_{4}, \boldsymbol{r}_{3}\right)\left[Q\left(t_{3}, t_{3}-t_{12}, \boldsymbol{n}_{3} ; t_{4}, \boldsymbol{n}_{4} ; \boldsymbol{r}_{3}\right) .\right. \\
\left.-\frac{i \pi}{2} g^{K}\left(t_{3}, t_{3}-t_{12} ; \boldsymbol{n}_{3}, \boldsymbol{r}_{3}\right) \int_{t_{3}}^{t_{3}} d t_{5} Q\left(t_{5}, t_{5}, \boldsymbol{n}_{3} ; t_{4}, \boldsymbol{n}_{4} ; \boldsymbol{r}_{3}\right)\right],
\end{gathered}
$$

where $t_{12}=t_{1}-t_{2}$ and the notation (5.2) is used ${ }^{39}$.

For future use, we note following properties [see also the discussion following Eq. (5.36)]:

$\delta g_{-}(t, t)=\delta g_{+}(t, t)=0 ; \int d \boldsymbol{n} \delta Q\left(t_{1}, t_{2}, \boldsymbol{n} ; t_{3}, \boldsymbol{n}_{1} ; \boldsymbol{r}\right)=0$

as one can see from Eqs. (5.77)-(5.78) and from the definitions (5.76d) and (5.27).

The canonical form of the kinetic equation is obtained by performing the time Wigner transform (5.63b) of both 
sides of Eq. (5.71). It is clear from the structure of the collision integrals that this procedure will lead to the appearance of Poisson brackets in the right hand side of the kinetic equation. We choose, however, another route: we will prove the existence of the conservation laws before the Wigner transform. It will enable us to argue that these Poisson brackets (in our formulation of the kinetic equation) give only small contributions, which can be neglected within the accuracy of the kinetic equation.

\section{F. Conservation laws}

The derivation of the conservation laws is based on the following properties of the collision integrals of the previous subsection

$$
\begin{aligned}
& \left\langle\widehat{\mathrm{St}}_{\tau} g\right\rangle_{\boldsymbol{n}}=0 \\
& \left\langle\widehat{\mathrm{St}}_{-}\right\rangle_{\boldsymbol{n}}=0 \\
& \left\langle\widehat{\mathrm{St}}_{1}^{\mathrm{el}}\right\rangle_{\boldsymbol{n}}=0
\end{aligned}
$$

The physical meaning of conditions (5.80) is that the corresponding terms in the collision integral conserve the number of particles within the energy shell. Equations (5.80) follow immediately from definitions (5.8), (5.75b), (5.76b), (5.76d) and (5.79).

The two remaining contributions to the collision integral have the properties

$$
\begin{aligned}
& \lim _{t_{1} \rightarrow t_{2}} \widehat{\mathrm{St}}_{+}\left(t_{1}, t_{2}\right)=0 ; \\
& \lim _{t_{1} \rightarrow t_{2}} \widehat{\mathrm{St}}_{1}^{\mathrm{in}}\left(t_{1}, t_{2}\right)=0,
\end{aligned}
$$

and

$$
\begin{aligned}
& \lim _{t_{1} \rightarrow t_{2}}\left(\partial_{t_{1}}-\partial_{t_{2}}\right) \widehat{\mathrm{St}}_{+}\left(t_{1}, t_{2}\right)=0 \\
& \lim _{t_{1} \rightarrow t_{2}}\left(\partial_{t_{1}}-\partial_{t_{2}}\right) \widehat{\mathrm{St}}_{1}^{\mathrm{in}}\left(t_{1}, t_{2}\right) \\
& =\frac{1}{4 \pi} \int \frac{d \boldsymbol{n}_{2} d \boldsymbol{n}_{3}}{\Omega_{d}^{2}} \gamma_{12}^{3}\left\{\left[\partial_{t} \mathcal{K}^{K} \partial_{t}\right]_{31}\left(t_{1}, t_{1}\right)\right. \\
& \quad-\frac{i \pi}{4} \int d t_{3}\left[\partial_{t} \mathcal{K}^{R}\right]_{13}\left(t_{1}, t_{3}\right)\left[g\left(t_{1}, t_{3}, \boldsymbol{n}_{1}\right) g\left(t_{3}, t_{1}, \boldsymbol{n}_{2}\right)\right. \\
& \left.\left.\quad+g\left(t_{1}, t_{3}, \boldsymbol{n}_{2}\right) g\left(t_{3}, t_{1}, \boldsymbol{n}_{1}\right)\right]-\left(\boldsymbol{n}_{1} \rightarrow \boldsymbol{n}_{2}\right)\right\}
\end{aligned}
$$

where we use the notation (5.74) and the vertex is defined in Eq. (5.28). Equations (5.81a) and (5.82a) immediately follow from the definition (5.76c) and the condition (5.79). Derivations of Eqs. (5.81b) and (5.82b) are presented in Appendix E.

Expressions (5.81) mean that while not conserving the number of particles for a given energy shell, the terms $\widehat{\mathrm{St}}_{+}$and $\widehat{\mathrm{St}}_{1}^{\mathrm{in}}$ conserve the total number of particles for a given direction (small angle inelastic scattering). Equation (5.82a) means that the inelastic $\widehat{\mathrm{St}}_{+}$term conserves not only the number of particles but also the energy for a given direction. Equation (5.82b) means $\widehat{\mathrm{St}}_{1}^{\text {in }}$ term does not conserve the energy for a given direction, thus describing the energy exchange between the quasiparticles and the electron-hole pairs discussed in Sec. V C.

The possibility to find the conserved energy current is based on a certain relation between Eq. (5.82b) and the collision integral for the electron-hole pairs - we now turn to the discussion of this relation.

We substitute Eqs. (5.55a) and (5.58) into Eq. (5.82b) and average the result over $\boldsymbol{n}$; then using the analytical property

$$
\mathcal{K}^{R}(t, t)=\int \frac{d \omega}{2 \pi} \mathcal{K}^{R}(\omega)=0
$$

together with $\mathcal{L}^{\rho}(1,2)=\overline{\mathcal{L}}^{\rho}(2,1), \mathcal{L}^{g}(1,2)=\overline{\mathcal{L}}^{g}(2,1)$, $\mathcal{N}^{\rho, g}(1,2)=\mathcal{N}^{\rho, g}(2,1)$ and Eq. (5.38), we find:

$$
\begin{aligned}
\lim _{t_{1} \rightarrow t_{2}}\left(\partial_{t_{1}}-\partial_{t_{2}}\right)\left\langle\widehat{\mathrm{St}}_{1}\right\rangle_{n} \\
=-\frac{i}{\pi \nu} \operatorname{Tr}_{\boldsymbol{n}}\left[\hat{\mathcal{L}}^{g}\left(\hat{\mathcal{N}}^{g} \widehat{\mathrm{St}}_{\tau}+\hat{M}\right)\right] \\
\quad+\frac{i}{\pi \nu} \operatorname{Tr}_{\boldsymbol{n}}\left[\hat{\mathcal{L}}^{\rho}\left(\hat{\mathcal{N}}^{\rho} \widehat{\mathrm{St}}_{\tau}+\hat{M}\right)\right],
\end{aligned}
$$

where $\widehat{\mathrm{St}}_{\tau}$ is defined in Eq. (5.8) and $\operatorname{Tr}_{\boldsymbol{n}}$ acts as

$$
\left[\operatorname{Tr}_{\boldsymbol{n}} \hat{A}\right](t, \boldsymbol{r}) \equiv \int \frac{d \boldsymbol{n}}{\Omega_{d}} A(t, \boldsymbol{n}, \boldsymbol{r} ; t, \boldsymbol{n}, \boldsymbol{r}) .
$$

The corresponding traces of the collision integrals for the electron-hole pairs - Eq. $(5.59 \mathrm{c})^{40}-$ are:

$$
\begin{aligned}
& \operatorname{Tr}_{\boldsymbol{n}} {\left[\hat{\mathcal{L}}^{g} \widehat{\mathrm{St}}^{g}\left\{\mathcal{N}^{g}, g\right\}\right]=\operatorname{Tr}_{\boldsymbol{n}}\left[\left[\hat{\mathcal{L}}^{g}, \widehat{\mathrm{St}}_{\tau}\right] \hat{\mathcal{N}}^{g}\right.} \\
&+\left.+2 \hat{\mathcal{L}}^{g}\left(\hat{\mathcal{N}}^{g} \widehat{\mathrm{St}}_{\tau}+\hat{M}\right)\right] \\
& \operatorname{Tr}_{\boldsymbol{n}}\left[\hat{\mathcal{L}}^{\rho} \widehat{\mathrm{St}}^{\rho}\left\{\mathcal{N}^{\rho}, g\right\}\right]=\operatorname{Tr}_{\boldsymbol{n}}\left[\left[\hat{\mathcal{L}}^{\rho}, \widehat{\mathrm{St}}_{\tau}\right] \hat{\mathcal{N}}^{\rho}\right. \\
&\left.\quad+2 \hat{\mathcal{L}}^{\rho}\left(\hat{\mathcal{N}}^{\rho} \widehat{\mathrm{St}}_{\tau}+\hat{M}\right)\right] .
\end{aligned}
$$

Comparing Eqs. (5.84) and (5.86) and using once again Eqs. (5.25) and (5.54), we arrive to the desired relation between the collision integrals:

$$
\begin{aligned}
& 2 i \pi \nu \lim _{t_{1} \rightarrow t_{2}}\left(\partial_{t_{1}}-\partial_{t_{2}}\right)\left\langle\widehat{\operatorname{St}}\left\{g, \mathcal{N}^{\rho}, \mathcal{N}^{g}\right\}\right\rangle_{\boldsymbol{n}} \\
& +\operatorname{Tr}_{\boldsymbol{n}}\left[\hat{\mathcal{L}}^{\rho} \widehat{\mathrm{St}}^{\rho}\left\{\mathcal{N}^{\rho}, g\right\}\right]-\operatorname{Tr}_{\boldsymbol{n}}\left[\hat{\mathcal{L}}^{g} \widehat{\mathrm{St}}^{g}\left\{\mathcal{N}^{g}, g\right\}\right] \\
& =\operatorname{Tr}_{\boldsymbol{n}}\left[\left[\boldsymbol{v} \cdot \boldsymbol{\nabla}, \hat{\mathcal{L}}^{g}\right] \hat{\mathcal{N}}^{g}-i\left[\hat{H}_{\mathrm{e}-\mathrm{h}}\left(i \partial_{t},-i \boldsymbol{\nabla}\right), \hat{\mathcal{L}}^{\rho}\right] \hat{\mathcal{N}}^{\rho}\right] .
\end{aligned}
$$

The left hand side of Eq. (5.87) is the quantum counterpart of the relation (2.3b) and Eqs. (5.80)-(5.81) are related to Eq. (2.3a); we will derive the expressions for the electric and energy currents in the spirit of our discussion in Sec. II A. 
We begin with the conservation of electric charge. According to Eqs. (5.4) and (5.6) the charge density is given by

$$
\rho(t, \boldsymbol{r})=-\frac{e \nu \pi}{2} \lim _{t_{1} \rightarrow t_{2} \rightarrow t}\left\langle g\left(t_{1}, t_{2}, \boldsymbol{n}, \boldsymbol{r}\right)\right\rangle_{\boldsymbol{n}} .
$$

Taking the limit $t_{1} \rightarrow t_{2} \rightarrow t$ in both sides of Eq. (5.71) and using Eqs. (5.72), (5.80) and (5.81), we obtain the continuity equation

$$
\partial_{t} \rho+\nabla \cdot \boldsymbol{j}=0
$$

where

$$
\boldsymbol{j}(t, \boldsymbol{r})=-\frac{e v_{F} \nu \pi}{2} \lim _{t_{1} \rightarrow t_{2} \rightarrow t}\left\langle\boldsymbol{n} g\left(t_{1}, t_{2}, \boldsymbol{n}, \boldsymbol{r}\right)\right\rangle_{\boldsymbol{n}},
$$

compare with Eqs. (2.5) - (2.7).

Having found the usual equation for the electric currents, we turn to the energy conservation. Acting with the operator $\left(\partial_{t_{1}}-\partial_{t_{2}}\right)$ on both sides of Eq. (5.71) and introducing the quantities

$$
\begin{aligned}
& u_{e}(t, \boldsymbol{r})=-\frac{i \pi \nu}{4} \lim _{t_{1} \rightarrow t_{2} \rightarrow t}\left(\partial_{t_{1}}-\partial_{t_{2}}\right)\left\langle g\left(t_{1}, t_{2}, \boldsymbol{n}, \boldsymbol{r}\right)\right\rangle_{\boldsymbol{n}} \\
& \boldsymbol{j}_{e}^{\epsilon}(t, \boldsymbol{r})=-\frac{i \pi \nu v_{F}}{4} \lim _{t_{1} \rightarrow t_{2} \rightarrow t}\left(\partial_{t_{1}}-\partial_{t_{2}}\right)\left\langle\boldsymbol{n} g\left(t_{1}, t_{2}, \boldsymbol{n}, \boldsymbol{r}\right)\right\rangle_{\boldsymbol{n}},
\end{aligned}
$$

we find

$$
\begin{aligned}
\partial_{t} u_{e} & +\nabla \cdot \boldsymbol{j}_{e}^{\epsilon}=\boldsymbol{j} \cdot \boldsymbol{E} \\
& +i \nu \pi \lim _{t_{1} \rightarrow t_{2} \rightarrow t}\left(\partial_{t_{1}}-\partial_{t_{2}}\right)\left\langle\widehat{\mathrm{St}}\left\{g, \mathcal{N}^{\rho}, \mathcal{N}^{g}\right\}\right\rangle_{\boldsymbol{n}} .
\end{aligned}
$$

The expression in the left hand side of Eq. (5.92) has the form of the continuity equation for the energy current of the electrons and the first term in the right hand side is the Joule heat acting as an energy source. The last term in the right-hand side indicates that the electron system by itself is open, due to the energy exchange with the electron-hole pairs. As we discussed in Sec. II A this means that in the definition of the conserved energy and energy current densities the contribution of these degrees of freedom must be taken into account. To accomplish this task, we premultiply Eqs. (5.59a) and (5.59b) by $\hat{\mathcal{L}}^{g}$ and $\hat{\mathcal{L}}^{\rho}$ respectively. Using Eqs. $(5.25),(5.54)$ and $(5.59 \mathrm{c})$ and taking the trace $\operatorname{Tr}_{n}$ [see Eq. (5.85)] of both sides, we obtain:

$$
\begin{aligned}
\partial_{t} u_{g}+\boldsymbol{\nabla} \cdot \boldsymbol{j}_{g}^{\epsilon} & -\frac{1}{2} \operatorname{Tr}_{\boldsymbol{n}}\left[\boldsymbol{v} \cdot \boldsymbol{\nabla}, \hat{\mathcal{L}}^{g}\right] \hat{\mathcal{N}}^{g} \\
& =\frac{1}{2} \operatorname{Tr}_{\boldsymbol{n}} \hat{\mathcal{L}}^{g} \widehat{\mathrm{St}}^{g} \\
\partial_{t} u_{\rho}+\boldsymbol{\nabla} \cdot \boldsymbol{j}_{\rho}^{\epsilon} & -\frac{i}{2} \operatorname{Tr}_{\boldsymbol{n}}\left[\hat{H}_{\mathrm{e}-\mathrm{h}}\left(i \partial_{t},-i \boldsymbol{\nabla}\right), \hat{\mathcal{L}}^{\rho}\right] \hat{\mathcal{N}}^{\rho} \\
& =\frac{1}{2} \operatorname{Tr}_{\boldsymbol{n}} \hat{\mathcal{L}}^{\rho} \widehat{\mathrm{St}}^{\rho}
\end{aligned}
$$

where the energy densities $u_{\rho, g}$ and currents $\boldsymbol{j}_{\rho, g}^{\epsilon}$ are defined as

$$
\begin{gathered}
u_{g}(t, \boldsymbol{r})=\frac{1}{2} \operatorname{Tr}_{\boldsymbol{n}} \hat{\mathcal{L}}^{g} \hat{\mathcal{N}}^{g} ; u_{\rho}(t, \boldsymbol{r})=\frac{1}{2} \operatorname{Tr}_{\boldsymbol{n}}\left\{\frac{1}{1+\hat{F}} ; \hat{\mathcal{L}}^{\rho} \hat{\mathcal{N}}^{\rho}\right\} \\
\boldsymbol{j}_{g}^{\epsilon}=\frac{1}{2} \operatorname{Tr}_{\boldsymbol{n}} \boldsymbol{v} \hat{\mathcal{L}}^{g} \hat{\mathcal{N}}^{g} ; \quad \boldsymbol{j}_{\rho}^{\epsilon}=\frac{1}{2} \operatorname{Tr}_{\boldsymbol{n}}\left\{\hat{\boldsymbol{s}} ; \hat{\mathcal{L}}^{\rho} \hat{\mathcal{N}}^{\rho}\right\}
\end{gathered}
$$

The velocity operator $\hat{\boldsymbol{s}}$ is defined in Eq. (5.65) and the notation (5.60) is used.

We now add Eq. (5.93a) to Eq. (5.92) and subtract Eq. (5.93b). According to Eq. (5.87) all the collision terms as well as the commutators cancel and we obtain the energy balance equation [compare with Eq. (2.8)]:

$$
\begin{aligned}
& \partial_{t} u_{t o t}+\nabla \cdot \boldsymbol{j}_{\text {tot }}^{\epsilon}=\boldsymbol{j} \cdot \boldsymbol{E} \\
& u_{t o t}(t, \boldsymbol{r})=u_{e}(t, \boldsymbol{r})+u_{\rho}(t, \boldsymbol{r})-u_{g}(t, \boldsymbol{r}) \\
& \boldsymbol{j}_{\text {tot }}^{\epsilon}(t, \boldsymbol{r})=\boldsymbol{j}_{e}^{\epsilon}(t, \boldsymbol{r})+\boldsymbol{j}_{\rho}^{\epsilon}(t, \boldsymbol{r})-\boldsymbol{j}_{g}^{\epsilon}(t, \boldsymbol{r})
\end{aligned}
$$

Equations (5.94), (5.91), (5.93c) and (5.93d) constitute the main result of this subsection. They define the conserved currents in terms of quantities to be found from the kinetic equations. It is important to emphasize that the conservation laws thus found are exact (at one loop) in the sense that no approximation has been made beyond the usual Fermi-liquid theory: in detail, no gradient or harmonic expansion has been made and no time or space (except those suppressed by a factor of $q / p_{F}$ ) Poisson brackets have been neglected yet.

Within the same accuracy with which the kinetic equations (5.64) were derived, performing the Wigner transforms (5.61)-(5.63) of Eq. (5.90), (5.91) and (5.93d) we find

$$
\boldsymbol{j}=e v_{F} \nu \int d \epsilon\langle\boldsymbol{n} f(\epsilon, \boldsymbol{n} ; t, \boldsymbol{r})\rangle_{\boldsymbol{n}}
$$

for the electric current density and

$$
\begin{aligned}
& \boldsymbol{j}_{e}^{\epsilon}=v_{F} \nu \int d \epsilon \epsilon\langle\boldsymbol{n} f(\epsilon, \boldsymbol{n} ; t, \boldsymbol{r})\rangle_{\boldsymbol{n}} \\
& \boldsymbol{j}_{\rho}^{\epsilon}=\int \frac{d \omega}{2 \pi} \omega \int \frac{d^{d} q}{(2 \pi)^{d}}\left\langle\left\{\hat{\boldsymbol{s}}(\omega, \boldsymbol{q}) ; \hat{\mathcal{L}}^{\rho}(\omega, \boldsymbol{q}) \hat{N}^{\rho}(\omega, \boldsymbol{q} ; t, \boldsymbol{r})\right\}\right\rangle_{\boldsymbol{n}} \\
& \boldsymbol{j}_{g}^{\epsilon}=\int \frac{d \omega}{2 \pi} \omega \int \frac{d^{d} q}{(2 \pi)^{d}}\left\langle\boldsymbol{v} \hat{\mathcal{L}}^{g}(\omega, \boldsymbol{q}) \hat{N}^{g}(\omega, \boldsymbol{q} ; t, \boldsymbol{r})\right\rangle_{\boldsymbol{n}}
\end{aligned}
$$

for the energy current densities, in agreement with Eqs. (3.14)-(3.15).

What remains to be done is to reduce the found expressions to the usual form of the quantum Boltzmann equation. This is the subject of the next subsection. 


\section{G. The quantum kinetic equation}

After the Wigner transforms (5.61)-(5.63b), Eq. (5.71) becomes

$$
\left[\partial_{t}+\boldsymbol{v} \cdot \boldsymbol{\nabla}+e \boldsymbol{v} \cdot \boldsymbol{E} \frac{\partial}{\partial \epsilon}\right] f(\epsilon, \boldsymbol{n} ; t, \boldsymbol{r})=\widehat{\operatorname{St}}\left\{f, N^{\rho}, N^{g}\right\} .
$$

The collision integral is the sum of the inelastic and elastic parts

$$
\widehat{\operatorname{St}}\left\{f, N^{\rho}, N^{g}\right\}=\widehat{\operatorname{St}}_{\text {in }}\left\{f, N^{\rho}, N^{g}\right\}+\widehat{\operatorname{St}}_{\mathrm{el}}\{f\} .
$$

The elastic part is obtained by adding the transform of the "bare" collision integral [the first term on the right hand side of Eq. (5.72)] to the transforms of $\widehat{\mathrm{St}}_{1}^{\mathrm{el}}$, Eq. (5.75c), and $\widehat{\mathrm{St}}_{-}, \mathrm{Eq} .(5.76 \mathrm{~b})$. The inelastic part is given by the transform of $\widehat{\mathrm{St}}_{1}$, Eq. $(5.75 \mathrm{~b})$, plus the transform of $\widehat{\mathrm{St}}_{+}$, Eq. (5.76c). However for the sake of compactness we will not make such a distinction between elastic and inelastic contributions and, using a notation resembling that of section $\mathrm{VE}$, we write the collision integral in the following form:

$$
\begin{aligned}
\widehat{\mathrm{St}}\left\{f, N^{\rho}, N^{g}\right\}= & \widehat{\mathrm{St}}_{\tau} f+\widehat{\mathrm{St}}_{1}\left\{f, N^{\rho}, N^{g}\right\} \\
& +\widehat{\mathrm{St}}_{-}\{f\}+\widehat{\mathrm{St}}_{+}\left\{f, N^{\rho}, N^{g}\right\},
\end{aligned}
$$

where the first term on the right hand side is the transform of the "bare" collision integral and the other terms are given below.

Keeping the elastic and inelastic parts of the collision integral (5.73) in a single formula, the corresponding contribution is obtained by first substituting Eqs. (5.58) and then performing the Wigner transforms (5.61)-(5.63) [and using their property (5.83)]. We decompose the result into distinct contributions due to the two bosonic degrees of freedom:

$$
\widehat{\mathrm{St}}_{1}(\epsilon, \boldsymbol{n} ; t, \boldsymbol{r})=\widehat{\mathrm{St}}_{1}^{e-\rho}(\epsilon, \boldsymbol{n} ; t, \boldsymbol{r})-\widehat{\mathrm{St}}_{1}^{e-g}(\epsilon, \boldsymbol{n} ; t, \boldsymbol{r})
$$

As usual the collisions with the "ghost" particles enter with the opposite sign. In terms of the combination (5.66) of distribution functions that we denoted by $\Upsilon$ and the vertex (5.28), these contributions are (we suppress the spectator arguments $t$ and $\boldsymbol{r}$ in both sides of the equations):

$$
\begin{aligned}
& {\widehat{\mathrm{St}_{1}}}^{e-\rho}\left(\epsilon, \boldsymbol{n}_{1}\right)=\frac{1}{\nu} \int \frac{d \omega}{2 \pi} \frac{1}{\omega} \int \frac{d^{d} q}{(2 \pi)^{d}} \int \frac{d \boldsymbol{n}_{2} d \boldsymbol{n}_{3} d \boldsymbol{n}_{4}}{\Omega_{d}^{3}} \\
& \times\left\{\gamma_{12}^{3}\left[\mathcal{L}_{34}^{\rho} \Upsilon_{41 ; 21}^{\rho}(\epsilon, \omega, \boldsymbol{q})+\Upsilon_{34 ; 21}^{\rho}(\epsilon, \omega, \boldsymbol{q}) \overline{\mathcal{L}}_{41}^{\rho}\right]\right. \\
& \left.+\gamma_{21}^{3}\left[\mathcal{L}_{34}^{\rho} \Upsilon_{42 ; 21}^{\rho}(\epsilon, \omega, \boldsymbol{q})+\Upsilon_{34 ; 21}^{\rho}(\epsilon, \omega, \boldsymbol{q}) \overline{\mathcal{L}}_{42}^{\rho}\right]\right\} \\
& \widehat{\mathrm{St}}_{1}^{e-g}\left(\epsilon, \boldsymbol{n}_{1}\right)=\frac{1}{\nu} \int \frac{d \omega}{2 \pi} \frac{1}{\omega} \int \frac{d^{d} q}{(2 \pi)^{d}} \int \frac{d \boldsymbol{n}_{2} d \boldsymbol{n}_{3} d \boldsymbol{n}_{4}}{\Omega_{d}^{3}} \\
& \times\left\{\gamma_{12}^{3}\left[\mathcal{L}_{34}^{g} \Upsilon_{41 ; 21}^{g}(\epsilon, \omega, \boldsymbol{q})+\Upsilon_{34 ; 21}^{g}(\epsilon, \omega, \boldsymbol{q}) \overline{\mathcal{L}}_{41}^{g}\right]\right. \\
& \left.+\gamma_{21}^{3}\left[\mathcal{L}_{34}^{\rho} \Upsilon_{42 ; 21}^{g}(\epsilon, \omega, \boldsymbol{q})+\Upsilon_{34 ; 21}^{g}(\epsilon, \omega, \boldsymbol{q}) \overline{\mathcal{L}}_{42}^{g}\right]\right\}
\end{aligned}
$$

Here and below the short hand notation

$$
\mathcal{L}_{i j}^{\alpha}=\mathcal{L}^{\alpha}\left(\omega, \boldsymbol{q} ; \boldsymbol{n}_{i}, \boldsymbol{n}_{j}\right), \alpha=g, \rho
$$

is used. It is readily seen that this contributions coincide with the local electron-boson collision integral of Eq. (3.19b).

Proceeding as above, the transform of Eq. (5.76b) is

$$
\begin{aligned}
& \widehat{\mathrm{St}}_{-}\left(\epsilon, \boldsymbol{n}_{1}\right)=\widehat{\mathrm{St}}_{e l}\left(\epsilon, \boldsymbol{n}_{1}\right)+\widehat{\mathrm{St}}_{-, l}\left(\epsilon, \boldsymbol{n}_{1}\right) \\
& \widehat{\mathrm{St}}_{e l}=\frac{2}{\nu} \operatorname{Re} \int \frac{d \omega}{2 \pi} \frac{1}{\omega} \int \frac{d^{d} q}{(2 \pi)^{d}} \int \frac{d \boldsymbol{n}_{2} \ldots d \boldsymbol{n}_{6}}{\Omega_{d}^{5}} \gamma_{13}^{2} \gamma_{46}^{5} \\
& \quad \times\left[\mathcal{L}^{\rho}-\mathcal{L}^{g}\right]_{52}\left[f\left(\epsilon-\omega, \boldsymbol{n}_{6}\right)-f\left(\epsilon-\omega, \boldsymbol{n}_{4}\right)\right] \\
& \quad \times\left[\mathcal{L}_{14}^{g} f\left(\epsilon, \boldsymbol{n}_{3}\right)+\mathcal{L}_{34}^{g} f\left(\epsilon, \boldsymbol{n}_{1}\right)\right] \\
& \widehat{\mathrm{St}}_{-, l}=\frac{1}{\nu} \int \frac{d \omega}{2 \pi} \frac{1}{\omega^{2}} \int \frac{d^{d} q}{(2 \pi)^{d}} \int \frac{d \boldsymbol{n}_{2} \ldots d \boldsymbol{n}_{6}}{\Omega_{d}^{5}} \gamma_{13}^{2} \gamma_{46}^{5} \\
& \times\left[2 f\left(\epsilon, \boldsymbol{n}_{4}\right)-f\left(\epsilon-\omega, \boldsymbol{n}_{4}\right)-f\left(\epsilon+\omega, \boldsymbol{n}_{4}\right)-\left(\boldsymbol{n}_{4} \rightarrow \boldsymbol{n}_{6}\right)\right] \\
& \times \int d \epsilon_{1}\left[f\left(\epsilon_{1}, \boldsymbol{n}_{1}\right)\left[1-f\left(\epsilon_{1}-\omega, \boldsymbol{n}_{3}\right)\right]+\left(\boldsymbol{n}_{1} \leftrightarrow \boldsymbol{n}_{3}\right)\right] \\
& \times\left[\mathcal{L}^{\rho}-\mathcal{L}^{g}\right]_{52} \mathcal{L}_{14}^{g}+\frac{1}{2}\left[\mathcal{L}^{\rho}-\mathcal{L}^{g}\right]_{52}\left[\mathcal{L}_{14}^{g}-\mathcal{L}_{34}^{g}\right]
\end{aligned}
$$

Equation (5.102b) is the (singlet part of the) elastic electron-electron collision integral, Eq. (3.20b). To obtain Eq. (5.102c) in the given form, we used the analytic properties of the propagators and performed the change of variable $\epsilon_{1} \rightarrow \epsilon_{1}+\omega$ in some of the terms.

Finally we perform the transform of Eq. (5.76c) and obtain:

$$
\widehat{\mathrm{St}}_{+}\left(\epsilon, \boldsymbol{n}_{1}\right)=\widehat{\mathrm{St}}_{n}^{e-e}\left(\epsilon, \boldsymbol{n}_{1}\right)+\widehat{\mathrm{St}}_{+, n}\left(\epsilon, \boldsymbol{n}_{1}\right)+\widehat{\mathrm{St}}_{+, l}\left(\epsilon, \boldsymbol{n}_{1}\right)
$$

The first term is given by (the singlet part of) Eq. (3.20d). The second term is

$$
\widehat{\mathrm{St}}_{+, n}=\widehat{\mathrm{St}}_{+, n}^{e-\rho}-\widehat{\mathrm{St}}_{+, n}^{e-g}
$$

with $\widehat{\mathrm{St}}_{+, n}^{e-\alpha}$ given by Eq. (3.19d) excluding the last line. The third term is:

$$
\begin{aligned}
& \widehat{\mathrm{St}}_{+, l}=\frac{1}{2 \nu} \int \frac{d \omega}{2 \pi} \frac{1}{\omega} \int \frac{d^{d} q}{(2 \pi)^{d}} \int \frac{d \boldsymbol{n}_{2} \ldots d \boldsymbol{n}_{6}}{\Omega_{d}^{5}} \gamma_{13}^{2} \gamma_{46}^{5} \\
& \times\left[2 f\left(\epsilon, \boldsymbol{n}_{4}\right)-f\left(\epsilon-\omega, \boldsymbol{n}_{4}\right)-f\left(\epsilon+\omega, \boldsymbol{n}_{4}\right)-\left(\boldsymbol{n}_{4} \rightarrow \boldsymbol{n}_{6}\right)\right] \\
& \times\left[\mathcal{L}^{\rho}\left(2 N^{\rho}+1\right)-\mathcal{L}^{g}\left(2 N^{g}+1\right)\right]_{52}\left[\mathcal{L}_{34}^{g}-\mathcal{L}_{14}^{g}\right] \quad(5.103 \mathrm{c})
\end{aligned}
$$

Adding Eqs. (5.103c) and (5.102c) we recover the last line of Eq. (3.19d) and the (the singlet part of the) local electron-electron collision integral, Eq. (3.20f). This concludes the derivation of the quantum kinetic equation for the singlet channel.

\section{H. The triplet channel}

The inclusion of the interaction in the triplet channel is straightforward; in the Eilenberger equation (5.7) we 
add to the left hand side the term:

$$
i[\hat{\boldsymbol{\phi}} \cdot \boldsymbol{\sigma} ; \hat{g}]
$$

where $\sigma_{i}$ are the Pauli matrices $[i=x, y, z]$ and the fluctuating field $\hat{\phi}_{i}$ is a 3 component vector in the $L=1$ angular momentum space. Therefore all the triplet channel propagators, the polarization operators and the density matrices should be treated as $3 \times 3$ matrices; for example we have

$$
\left[D_{0}\right]_{i j}(1,2)=-\frac{F^{\sigma}\left(\theta_{12}\right) \delta\left(\boldsymbol{r}_{12}\right)}{\nu} \delta\left(t_{12}\right) \delta_{i j}
$$

[cf. Eq. (5.15)] and the retarded polarization operator is given by

$$
\Pi_{i j}^{R}(1,2)=\nu\left\{\delta_{12}+\frac{\pi}{4} \operatorname{tr}\left[\sigma_{i} \frac{\delta g^{K}\left(t_{1}, t_{1}, \boldsymbol{n}_{1}, \boldsymbol{r}_{1}\right)}{\delta \phi_{+j}\left(t_{2}, \boldsymbol{r}_{2}, \boldsymbol{n}_{2}\right)}\right]\right\},
$$

where the trace is over spin indices.

The trace of triplet channel operators includes the sum over the indices $i, j$. In the absence of the magnetic field, all the operators are diagonal, e.g.:

$$
\left[\mathcal{L}^{\sigma}\right]_{i j}=\mathcal{L}^{\sigma} \delta_{i j}
$$

and the trace results in extra factors of 3 in comparison to the singlet channel. The derivation can therefore be repeated with simple modifications and it gives the quantum kinetic equation presented in Sec. III. We note only one main difference in the derivation for the triplet channel: the gauge transformation, which has the form:

$$
\hat{g} \rightarrow e^{-i \hat{\boldsymbol{K}} \cdot \boldsymbol{\sigma}} \hat{g} e^{i \hat{\boldsymbol{K}} \cdot \boldsymbol{\sigma}},
$$

does not commute with the interaction term (5.104). Additional second order terms arise due to commutators of the Pauli matrices; however these terms vanish in the one loop approximation and we can neglect them.

In the next section we will use the quantum kinetic equation to calculate the interaction corrections to transport coefficients and specific heat.

\section{DERIVATION OF TRANSPORT COEFFICIENTS AND SPECIFIC HEAT}

In this section the calculation of the transport coefficients for quasi one-dimensional, two-dimensional and three-dimensional systems is presented; the evaluation of the interaction correction to the specific heat is in the final subsection.

In order to calculate the currents in the presence of an external field (electric field $\boldsymbol{E}$ or temperature gradient $\boldsymbol{\nabla} T$ ), we need to solve the kinetic equations. We assume that the external fields are weak, i.e.

$$
e E L_{T} \ll T, \quad \nabla T L_{T} \ll T
$$

and the temperature length was defined in Eq. (5.53). These conditions ensure that the deviations from the equilibrium distribution functions are small and we can solve the equations by iteration.

In the lowest order, the distribution functions should null the collision integrals in Eqs. (3.4) and (3.6), and the sought corrections $\delta f, \delta N^{\alpha}$ are linear in the electric field or in the gradients of the distribution functions. In other words, we look for a solution to those equations of the form:

$$
\begin{aligned}
& f(\epsilon, \boldsymbol{n} ; \boldsymbol{r})=f_{F}(\epsilon ; \boldsymbol{r})+\delta f(\epsilon, \boldsymbol{n} ; \boldsymbol{r}) \\
& \hat{N}^{\alpha}(\omega, \boldsymbol{q} ; \boldsymbol{r})=N_{P}(\omega ; \boldsymbol{r}) \hat{\mathbb{1}}+\delta \hat{N}^{\alpha}(\omega, \boldsymbol{q} ; \boldsymbol{r}) ;
\end{aligned}
$$

where the Fermi and Plank distribution functions (2.2) depend on the spatial coordinate only through the temperature $T(\boldsymbol{r})$. For compactness, we will consider explicitly the singlet channel only and we will indicate how to include the triplet channel.

Let us start from the electronic part of the kinetic equation. The bare impurity collision part $\widehat{\mathrm{St}}_{\tau}$ is larger than the other terms, thus it suffices to calculate the latter in the first order of perturbation theory. Considering short range impurities, so that $\tau$ is independent of the scattering angle, we find:

$$
\begin{aligned}
& \delta f=\delta f_{0}+\delta f_{1} \\
& \delta f_{0}=\tau \boldsymbol{v} \cdot\left(e \boldsymbol{E}-\frac{\epsilon \boldsymbol{\nabla} T}{T}\right)\left(-\frac{\partial f_{F}(\epsilon)}{\partial \epsilon}\right) \\
& \delta f_{1}=\tau \delta \widehat{\mathrm{St}}\left\{f_{F}+\delta f_{0}, N_{P}+\delta N^{\rho}, N_{P}+\delta N^{g}\right\}
\end{aligned}
$$

where $\delta \widehat{\mathrm{St}}$ is the linearized collision integral. Note that according to the discussion of the conservation laws in Sec. III, we need to consider only the local electronboson contribution, Eq. (3.19b), and the elastic electronelectron one, Eq. (3.20b).

Expression (6.2) is to be substituted in Eqs. (3.14) and $(3.15 \mathrm{~b})$ to find the electric current and the electron component of the energy current. Integration of the $\delta f_{0}$ term is straightforward. Due to the structure of the collision integrals (3.19)-(3.20), the integration over $\epsilon$ can be performed before the $\omega$ and $\boldsymbol{q}$ integrations in the $\delta f_{1}$ term. For the combination of distribution functions entering into Eq. (3.16a), we find

$$
\begin{aligned}
& \delta \Upsilon_{i j, k l}(\epsilon, \omega)=\delta N\left(\omega, \boldsymbol{q} ; \boldsymbol{n}_{i}, \boldsymbol{n}_{j}\right)\left\{f_{F}(\epsilon)-f_{F}(\epsilon-\omega)\right\} \\
& +\Omega_{d} \delta\left(\widehat{\boldsymbol{n}_{i} \boldsymbol{n}_{j}}\right) N_{P}(\omega)\left\{\delta f\left(\epsilon, \boldsymbol{n}_{k}\right)-\delta f\left(\epsilon-\omega, \boldsymbol{n}_{k}\right)\right\} \\
& +\Omega_{d} \delta\left(\widehat{\boldsymbol{n}_{i} \boldsymbol{n}_{j}}\right)\left[\delta f\left(\epsilon, \boldsymbol{n}_{l}\right)\left(1-f_{F}(\epsilon-\omega)\right)\right. \\
& \left.\quad-f_{F}(\epsilon) \delta f\left(\epsilon-\omega, \boldsymbol{n}_{k}\right)\right],
\end{aligned}
$$

which with the help of the identities

$$
\begin{aligned}
& \int d \epsilon f_{F}(\epsilon) \frac{\partial f_{F}(\epsilon-\omega)}{\partial \epsilon}=\frac{\partial}{\partial \omega}\left[\omega N_{P}(\omega)\right] \\
& \int d \epsilon \frac{\partial f_{F}(\epsilon)}{\partial \epsilon}\left[1-f_{F}(\epsilon-\omega)\right]=\frac{\partial}{\partial \omega}\left[\omega N_{P}(\omega)\right]
\end{aligned}
$$




$$
\begin{gathered}
\int d \epsilon \epsilon \frac{\partial f_{F}(\epsilon)}{\partial \epsilon}\left[1-f_{F}(\epsilon-\omega)\right]=\frac{1}{2} \omega^{2} \frac{\partial N_{P}(\omega)}{\partial \omega} ; \\
\int d \epsilon(\epsilon-\omega) \frac{\partial f_{F}(\epsilon-\omega)}{\partial \epsilon} f_{F}(\epsilon)=-\frac{1}{2} \omega^{2} \frac{\partial N_{P}(\omega)}{\partial \omega} ; \\
\int d \epsilon \epsilon^{2} \frac{\partial f_{F}(\epsilon)}{\partial \epsilon}\left[1-f_{F}(\epsilon-\omega)\right] \\
=\frac{\pi^{2}}{3} T^{2} \frac{\partial}{\partial \omega}\left[\omega N_{P}(\omega)\right]+\frac{1}{3} \omega^{3} \frac{\partial N_{P}(\omega)}{\partial \omega} \\
\int d \epsilon \epsilon(\epsilon-\omega) f_{F}(\epsilon) \frac{\partial f_{F}(\epsilon-\omega)}{\partial \epsilon} \\
=\frac{\pi^{2}}{3} T^{2} \frac{\partial}{\partial \omega}\left[\omega N_{P}(\omega)\right]-\frac{1}{6} \omega^{3} \frac{\partial N_{P}(\omega)}{\partial \omega}
\end{gathered}
$$

yields

$$
\begin{aligned}
& \frac{1}{2} \int d \epsilon\left[\delta \Upsilon_{i j, k l}(\epsilon, \omega, \boldsymbol{q})-\delta \Upsilon_{i j, k l}(\epsilon,-\omega,-\boldsymbol{q})\right] \\
& =e v_{F} \tau \boldsymbol{E} \cdot\left(\boldsymbol{n}_{k}-\boldsymbol{n}_{l}\right) \frac{\partial}{\partial \omega}\left(\omega N_{P}^{i j}\right) ; \\
& \frac{1}{2} \int d \epsilon \epsilon\left[\delta \Upsilon_{i j, k l}(\epsilon, \omega, \boldsymbol{q})-\delta \Upsilon_{i j, k l}(\epsilon,-\omega,-\boldsymbol{q})\right] \\
& =-\frac{\omega^{2}}{2} \delta N\left(\omega, \boldsymbol{q} ; \boldsymbol{n}_{i}, \boldsymbol{n}_{j}\right)-\frac{v_{F} \tau \boldsymbol{\nabla} T}{4 T} \cdot\left(\boldsymbol{n}_{l}+\boldsymbol{n}_{k}\right) \omega^{3} \frac{\partial N_{P}^{i j}}{\partial \omega} \\
& \quad+\frac{v_{F} \tau \boldsymbol{\nabla} T}{T} \cdot\left(\boldsymbol{n}_{l}-\boldsymbol{n}_{k}\right)\left[\frac{\pi^{2} T^{2}}{3} \frac{\partial\left(\omega N_{P}^{i j}\right)}{\partial \omega}+\frac{\omega^{3}}{12} \frac{\partial N_{P}^{i j}}{\partial \omega}\right],
\end{aligned}
$$

where

$$
N_{P}^{i j} \equiv\left[\hat{N}_{P}\right]\left(\boldsymbol{n}_{i}, \boldsymbol{n}_{j}\right) \equiv N_{P}(\omega) \Omega_{d} \delta\left(\widehat{\boldsymbol{n}_{i} \boldsymbol{n}_{j}}\right) .
$$

Here we retained only the odd in $\omega$ contribution because the even part vanishes after the $\omega$ integration in the relevant collision integral, see Eq. (3.19b).

The combination of the distribution functions entering the elastic collision part, Eq. (3.20b), gives

$$
\begin{aligned}
& \frac{1}{2} \int d \epsilon f_{F}(\epsilon)[\delta f(\epsilon-\omega, \boldsymbol{n})-\delta f(\epsilon+\omega, \boldsymbol{n})] \\
& =-e v_{F} \tau \boldsymbol{E} \cdot \boldsymbol{n} \frac{\partial}{\partial \omega}\left[\omega N_{P}(\omega)\right] . \\
& \frac{1}{2} \int d \epsilon \epsilon f_{F}(\epsilon)[\delta f(\epsilon-\omega, \boldsymbol{n})-\delta f(\epsilon+\omega, \boldsymbol{n})] \\
& =\frac{v_{F} \tau \boldsymbol{n} \cdot \boldsymbol{\nabla} T}{T}\left[\frac{\pi^{2} T^{2}}{3} \frac{\partial\left(\omega N_{P}(\omega)\right)}{\partial \omega}-\frac{\omega^{3}}{6} \frac{\partial N_{P}(\omega)}{\partial \omega}\right],
\end{aligned}
$$

where once again we retained only the odd in $\omega$ part, non-vanishing after the $\omega$ integration in Eq. (3.20b).

Using Eq. (3.14), we find the electric current $\boldsymbol{j}=\hat{\sigma} \boldsymbol{E}$ with the conductivity tensor $\hat{\sigma}$ given by:

$$
\hat{\sigma}=\sigma_{D}\left\{\hat{\mathbb{1}}+\int d \omega\left[\hat{\mathcal{S}}^{e l}(\omega)+\hat{\mathcal{E}}(\omega)\right] \frac{\partial}{\partial \omega}\left[\omega N_{P}(\omega)\right]\right\}
$$

where $\sigma_{D}=\tau v_{F}^{2} e^{2} \nu / d$ is the Drude conductivity. Indicating spatial indices with $\mu, \nu=1, \ldots, d$, the elastic kernels $\mathcal{S}_{\mu \nu}^{e l}$ and $\mathcal{E}_{\mu \nu}$, which originate from Eq. (3.19b) and Eq. (3.20b) respectively, are:

$$
\begin{aligned}
& \mathcal{S}_{\mu \nu}^{e l}(\omega)=\mathcal{S}_{\mu \nu}^{11}(\omega)+\mathcal{S}_{\mu \nu}^{12}(\omega) \\
& \mathcal{S}_{\mu \nu}^{11}(\omega)=\frac{d}{\pi \omega \nu} \int \frac{d^{d} q}{(2 \pi)^{d}} \int \frac{d \boldsymbol{n}_{1} d \boldsymbol{n}_{2}}{\left(\Omega_{d}\right)^{2}} n_{1 \mu} n_{1 \nu} \\
& \operatorname{Re}\left[\mathcal{L}_{11}^{\rho}-\mathcal{L}_{11}^{g}\right]+\operatorname{Re}\left[\mathcal{L}_{22}^{\rho}-\mathcal{L}_{22}^{g}\right]-2 \operatorname{Re}\left[\mathcal{L}_{12}^{\rho}-\mathcal{L}_{12}^{g}\right] \\
& \mathcal{S}_{\mu \nu}^{12}(\omega)=\frac{2 d}{\pi \omega \nu} \int \frac{d^{d} q}{(2 \pi)^{d}} \int \frac{d \boldsymbol{n}_{1} d \boldsymbol{n}_{2}}{\left(\Omega_{d}\right)^{2}} n_{1 \mu} n_{2 \nu} \operatorname{Re}\left[\mathcal{L}_{12}^{\rho}-\mathcal{L}_{12}^{g}\right] \\
& \mathcal{E}_{\mu \nu}(\omega)=-\frac{d \tau}{\pi \omega \nu} \int \frac{d^{d} q}{(2 \pi)^{d}} \int \frac{d \boldsymbol{n}_{1} \ldots d \boldsymbol{n}_{6}}{\left(\Omega_{d}\right)^{6}} \gamma_{12}^{6} \gamma_{43}^{5} \\
&\left(n_{1 \mu} n_{3 \nu}-n_{2 \mu} n_{3 \nu}\right) \operatorname{Re}\left\{\left[\mathcal{L}_{56}^{\rho}-\mathcal{L}_{56}^{g}\right]\left[\mathcal{L}_{13}^{g}+\mathcal{L}_{14}^{g}\right]\right\}
\end{aligned}
$$

where for compactness we kept the singlet channel correction only; inclusion of the triplet channel contribution is straightforward ${ }^{41}$. We show in appendix $\mathrm{F}$ that our expression for the conductivity coincides with the one of Ref. 17. It is natural that the conductivity does not involve any bosonic distribution function [cf. Eq. (6.5a)], because the inelastic electron collision with such bosons changes the energy of the electron but not the direction of its motion.

In contrast, even the electron contribution to the thermal conductivity tensor $\hat{\kappa}$, such that $\boldsymbol{j}_{\text {tot }}^{\epsilon}=-\hat{\kappa} \boldsymbol{\nabla} T$, is sensitive to the bosonic distribution functions. We represent the total thermal conductivity as

$$
\hat{\kappa}=\hat{\kappa}_{W F}+\delta \hat{\kappa}+\hat{\kappa}^{\rho}-\hat{\kappa}^{g}
$$

The first term in this expression obeys Wiedemann-Franz law even with the interaction to the conductivity included, i.e. $\hat{\kappa}_{W F}=\mathrm{L} \hat{\sigma} T$ with the Lorentz number given by Eq. (1.1). The second term represent the (electronic) correction to the Wiedemann-Franz due to the energy dependence of the elastic scattering and due to the inelastic electron scattering on the bosons. Finally, the third and the fourth term represent the contribution of the $\rho$ and $g$ bosons to the thermal transport. These additional contributions are given by:

$$
\begin{aligned}
\delta \hat{\kappa} & =\delta \hat{\kappa}_{e l}+\delta \hat{\kappa}_{i n} ; \\
{\left[\delta \kappa_{e l}\right]_{\mu \nu} } & =\frac{\sigma_{D}}{e^{2} T} \int d \omega\left[\mathcal{S}_{\mu \nu}^{e l}(\omega)-2 \mathcal{E}_{\mu \nu}(\omega)\right]\left[\frac{\omega^{3}}{12} \frac{\partial N_{P}}{\partial \omega}\right] ; \\
{\left[\delta \kappa_{i n}\right]_{\mu \nu} } & =\frac{\sigma_{D}}{e^{2} T} \int d \omega\left[\mathcal{S}_{\mu \nu}^{12}(\omega)-\mathcal{S}_{\mu \nu}^{11}(\omega)\right]\left[\frac{\omega^{3}}{4} \frac{\partial N_{P}}{\partial \omega}\right] \\
& +v_{F} \int \frac{d \omega}{2 \pi} \omega \int \frac{d^{d} q}{(2 \pi)^{d}} \int \frac{d \boldsymbol{n}_{1} d \boldsymbol{n}_{2} d \boldsymbol{n}_{3}}{\left(\Omega_{d}\right)^{3}} n_{1 \mu} \\
& \left\{\operatorname{Re}\left[\mathcal{L}_{12}^{\rho} \delta_{\nu} N_{23}^{\rho}+\mathcal{L}_{32}^{\rho} \delta_{\nu} N_{21}^{\rho}-\mathcal{L}_{12}^{\rho} \delta_{\nu} N_{21}^{\rho}\right]\right. \\
& \left.-\operatorname{Re}\left[\mathcal{L}_{12}^{g} \delta_{\nu} N_{23}^{g}+\mathcal{L}_{32}^{g} \delta_{\nu} N_{21}^{g}-\mathcal{L}_{12}^{g} \delta_{\nu} N_{21}^{g}\right]\right\}
\end{aligned}
$$




$$
\kappa_{\mu \nu}^{\alpha}=-\int \frac{d \omega}{2 \pi} \omega \int \frac{d^{d} q}{(2 \pi)^{d}} \int \frac{d \boldsymbol{n}_{1} d \boldsymbol{n}_{2}}{\left(\Omega_{d}\right)^{2}}\left\{\hat{s}_{\mu}^{\alpha} ; \mathcal{L}_{12}^{\alpha} \delta_{\nu} N_{21}^{\alpha}\right\}
$$

with

$$
\delta_{\mu} N_{i j}^{\alpha}=\frac{\delta}{\delta\left(\nabla_{\mu} T\right)}\left[\delta N^{\alpha}\left(\omega, \boldsymbol{q} ; \boldsymbol{n}_{i}, \boldsymbol{n}_{j}\right)\right] .
$$

Equations (6.8)-(6.12) are the complete expressions for the electric and thermal transport coefficients. To obtain the explicit result one has to solve Eqs. (3.6) to find the distribution functions $\delta N^{\alpha}$. We will do so by restricting ourselves to the diffusive $T \tau \ll 1$ regime, except for twodimensional systems for which we will consider the arbitrary temperature range ${ }^{42}$. Moreover for the Coulomb interaction we will consider the unitary limit (for infrared finite momentum integrals), which will enable us to drop all the terms that depend on $\partial_{\boldsymbol{q}} \hat{F}$.

From now on we will retain only the zeroth harmonic of the Fermi liquid constants, which we denote symbolically with $F_{0}^{\alpha}$. For the singlet channel this means

$$
F_{0}^{\rho} \equiv \int \frac{d \theta}{2 \pi} F^{\rho}(\theta)+\nu V(\boldsymbol{q})
$$

and for the triplet channel

$$
F_{0}^{\sigma} \equiv \int \frac{d \theta}{2 \pi} F^{\sigma}(\theta)
$$

We remind that $F^{g}=0$ and that for the Coulomb interaction the potential is:

$$
V(\boldsymbol{q})=\left\{\begin{array}{cc}
\frac{4 \pi e^{2}}{q^{2}}, & d=3 \\
\frac{2 \pi e^{2}}{q}, & d=2 \\
e^{2} \ln \frac{1}{(q a)^{2}}, & d=1
\end{array}\right.
$$

where $a$ is a length of the order of the quasi onedimensional wire width.

\section{A. Diffusive regime}

We consider first the distribution function $N^{g}$; substituting expression (6.1) into Eq. (3.6) we obtain at linear order in $\nabla T$

$$
\begin{array}{r}
-\frac{\omega}{T} \boldsymbol{v}_{1} \cdot \boldsymbol{\nabla} T \frac{\partial N_{P}^{12}(\omega)}{\partial \omega}+i\left[\boldsymbol{v} \cdot \boldsymbol{q} ; \delta N^{g}\right]=2\left\{\widehat{\mathrm{St}}_{\tau} ; \delta N^{g}\right\} \\
+v_{F} \frac{\omega}{T} \frac{\partial N_{P}(\omega)}{\partial \omega} \boldsymbol{\nabla} T \cdot\left(\Omega_{d} \delta\left(\widehat{\boldsymbol{n}_{1} \boldsymbol{n}_{2}}\right) \boldsymbol{n}_{1}-\boldsymbol{n}_{1}-\boldsymbol{n}_{2}\right)
\end{array}
$$

with $N_{P}^{i j}$ defined in Eq. (6.6). The (exact at this order) solution for $\delta N^{g}\left(\omega ; \boldsymbol{n}_{1}, \boldsymbol{n}_{2}\right)$ is:

$$
\delta N^{g}=\delta N^{0} \equiv v_{F} \tau \boldsymbol{n}_{1} \cdot \nabla T \frac{\omega}{T} \frac{\partial N_{P}^{12}(\omega)}{\partial \omega}
$$

For the distribution function $N^{\rho}$, the above is only the starting point for the iterative solution:

$$
\delta N^{\rho}=\delta N^{0}+\delta N^{1}
$$

The equation for $\delta N^{1}$ is:

$$
\begin{aligned}
& \left(-\frac{\omega}{T} \frac{\partial \hat{N}_{P}(\omega)}{\partial \omega} \nabla T\right) \cdot\left(\omega \frac{\partial}{\partial \boldsymbol{q}} \frac{\hat{F}^{\rho}}{1+\hat{F}^{\rho}}\right) \\
& +i\left[\omega \frac{\hat{F}^{\rho}}{1+\hat{F}^{\rho}} ; \delta \hat{N}^{g}\right]+i\left[\hat{H}_{\mathrm{e}-\mathrm{h}} ; \delta \hat{N}^{1}\right]=2\left\{\widehat{\mathrm{St}}_{\tau} ; \delta \hat{N}^{1}\right\} .
\end{aligned}
$$

In the diffusive limit $T \tau \ll 1$ the (first iteration) solution would be of the form

$$
\delta N^{1} \simeq \omega \tau\left(v_{F} \tau \frac{\omega}{T} \frac{\partial N_{P}(\omega)}{\partial \omega} \nabla T\right) \cdot \boldsymbol{V}
$$

for a vector $\boldsymbol{V}$ with magnitude of order one. However contributions from frequency $\omega$ larger than temperature $T$ are exponentially suppressed, i.e. $\omega \tau \lesssim T \tau \ll 1$; therefore $\delta N^{1}$ can be neglected in comparison to $\delta N^{g}$. Thus in the diffusive limit

$$
\delta_{\mu} N_{i j}^{\alpha}=v_{F} \tau \boldsymbol{n}_{i \mu} \frac{\omega}{T} \frac{\partial N_{P}^{i j}(\omega)}{\partial \omega} .
$$

For the propagators $\mathcal{L}^{\alpha}$ the diffusive approximation amounts to the substitution

$$
n_{\mu} n_{\nu} \rightarrow \frac{\delta_{\mu \nu}}{d}
$$

which leads to the following expression:

$$
\begin{aligned}
\mathcal{L}^{\alpha}\left(\boldsymbol{n}_{1}, \boldsymbol{n}_{2}\right)= & \tau\left(\delta\left(\widehat{\boldsymbol{n}_{1} \boldsymbol{n}_{2}}\right)-1\right)+L_{0}^{\alpha} \\
& +\left(n_{1}+n_{2}\right)_{\mu} L_{1 \mu}^{\alpha}+n_{1 \mu} n_{2 \nu} L_{2 \mu \nu}^{\alpha},
\end{aligned}
$$

where the functions $L_{i}^{\alpha}$ depend on $\omega, \boldsymbol{q}$ only and are explicitly:

$$
\begin{aligned}
L_{0}^{\alpha} & =\frac{1}{\frac{-i \omega}{1+F_{0}^{\alpha}}+D q^{2}} \\
L_{1 \mu}^{\alpha} & =-i \tau v_{F} q_{\mu} L_{0}^{\alpha} \\
L_{2 \mu \nu}^{\alpha} & =-d \tau D q_{\mu} q_{\nu} L_{0}^{\alpha} .
\end{aligned}
$$

Here $D=\tau v_{F}^{2} / d$ is the diffusion constant. These formulas are valid for $\omega, D q^{2} \ll 1 / \tau$.

Within this approximation, we have

$$
\mathcal{S}_{\mu \nu}^{12}(\omega) \pm \mathcal{S}_{\mu \nu}^{11}(\omega) \propto L_{2 \mu \nu}^{\rho}-L_{2 \mu \nu}^{g} .
$$

This means that in both $\hat{\sigma}$ and $\delta \hat{\kappa}-$ see Eqs. (6.8) and $(6.11)$ - we can neglect the contributions of the $\hat{\mathcal{S}}$ kernels [note that by inserting the solution (6.19) into Eq. (6.11c), $\delta \hat{\kappa}_{i n}$ is given by twice the first line of that equation]: indeed the leading contribution is given by the 
kernel $\hat{\mathcal{E}}$ in Eq. (6.11b). This kernel has the approximate form

$$
\mathcal{E}_{\mu \nu}(\omega)=\frac{4}{\pi \nu \tau} \frac{1}{d \omega} \int \frac{d^{d} q}{(2 \pi)^{d}} \operatorname{Re}\left[L_{0}^{g}\left(L_{2 \mu \nu}^{\rho}-L_{2 \mu \nu}^{g}\right)\right] .
$$

Finally the bosonic contributions [cf. Eq. (6.12)] to the thermal conductivity (6.10) can be written as ${ }^{43}$

$$
\hat{\kappa}^{\rho}-\hat{\kappa}^{g}=\frac{\sigma_{D}}{e^{2} T} \int d \omega \hat{\mathcal{B}}(\omega)\left[\frac{\omega^{3}}{4} \frac{\partial N_{P}}{\partial \omega}\right]
$$

with

$$
\mathcal{B}_{\mu \nu}(\omega)=-\frac{2 \delta_{\mu \nu}}{\pi \omega \nu} \int \frac{d^{d} q}{(2 \pi)^{d}} \operatorname{Re}\left[L_{0}^{\rho}-L_{0}^{g}\right] .
$$

The next step is to evaluate the momentum integrals; first we give the results for the short range interaction described by the (zeroth harmonic of the) Fermi-liquid constant $F_{0}$ and then we indicate the modifications needed to account for the long range part of the Coulomb interaction in the singlet channel. The triplet channel contributions are obtained by multiplying the found results by three and identifying $F_{0}$ with $F_{0}^{\sigma}$.

For the elastic kernel we find:

$$
\begin{aligned}
\mathcal{E}_{\mu \nu}(\omega)= & \frac{\delta_{\mu \nu}}{d} \frac{e^{2}}{\sigma_{D}} \frac{\Omega_{d}}{(2 \pi)^{d}}\left(\frac{|\omega|}{D}\right)^{\frac{d}{2}-1} \frac{1}{\omega} \\
& \times \frac{1}{\cos \frac{\pi d}{4}}\left[\frac{d}{2}-\frac{1}{F_{0}}\left(1+F_{0}-\left(1+F_{0}\right)^{1-\frac{d}{2}}\right)\right]
\end{aligned}
$$

The expression for the Coulomb interaction is obtained by taking the unitary limit $F_{0} \rightarrow+\infty$.

The result for the bosonic kernel is:

$$
\begin{aligned}
\mathcal{B}_{\mu \nu}(\omega)=\frac{\delta_{\mu \nu}}{2} & \frac{e^{2}}{\sigma_{D}} \frac{\Omega_{d}}{(2 \pi)^{d}}\left(\frac{|\omega|}{D}\right)^{\frac{d}{2}-1} \frac{1}{\omega} \\
& \times \frac{1}{\cos \frac{\pi d}{4}}\left[1-\left(1+F_{0}\right)^{1-\frac{d}{2}}\right]
\end{aligned}
$$

In $d=3$ the limit $F_{0} \rightarrow+\infty$ gives the correct formula for the long range contribution, but for $d=1$ the limit diverges. By retaining the full form of the interaction potential however such infrared divergence is cut off at the inverse of the screening radius. With logarithmic accuracy the result for the Coulomb interaction is found by substituting $[\ldots] \rightarrow-a k \ln ^{1 / 2}\left(D k^{2} /|\omega|\right)$, where $a$ is a length of the order of the wire width and $k^{2}=4 \pi e^{2} \nu$ is the square of the inverse screening radius [in the bulk].

We can now proceed with the calculation of the integrals over $\omega$ in Eqs. (6.8), (6.11) and (6.23) using the identity:

$$
\int d \omega \omega^{m} \partial_{\omega} N_{P}(\omega)=-2 T^{m} \zeta(m) \Gamma(m+1)
$$

Here $\zeta(x)$ is the Riemann zeta function, whose values at the points relevant for our discussion are:

$$
\begin{aligned}
& \zeta\left(-\frac{1}{2}\right) \approx-0.208, \quad \zeta(0)=-\frac{1}{2}, \\
& \zeta\left(\frac{1}{2}\right) \approx-1.460, \quad \zeta\left(\frac{3}{2}\right) \approx 2.612, \\
& \zeta(2)=\frac{\pi^{2}}{6} \approx 1.645, \quad \zeta\left(\frac{5}{2}\right) \approx 1.341,
\end{aligned}
$$

and $\Gamma(x)$ is the Euler gamma function, with values

$$
\begin{array}{ll}
\Gamma\left(\frac{1}{2}\right)=\sqrt{\pi}, & \Gamma(1)=1, \\
\Gamma\left(\frac{3}{2}\right)=\frac{1}{2} \sqrt{\pi}, & \Gamma\left(\frac{5}{2}\right)=\frac{3}{4} \sqrt{\pi}, \\
\Gamma(3)=2 \quad, & \Gamma\left(\frac{7}{2}\right)=\frac{15}{8} \sqrt{\pi} .
\end{array}
$$

Performing the final $\omega$ integrations we obtain:

$$
\begin{aligned}
\sigma= & \sigma_{D}+e^{2} \frac{\Omega_{d}}{(2 \pi)^{d}} \frac{2}{d} \zeta\left(\frac{d}{2}-1\right)\left(\frac{T}{D}\right)^{\frac{d}{2}-1} \Gamma\left(\frac{d}{2}\right) \\
& \times \frac{d-4}{2-d} \frac{1}{\cos \frac{\pi d}{4}}\left[\frac{d}{2}-\frac{1}{F_{0}}\left(1+F_{0}-\left(1+F_{0}\right)^{1-\frac{d}{2}}\right)\right]
\end{aligned}
$$

$$
\begin{aligned}
\delta \kappa=\frac{1}{3 d} & \frac{\Omega_{d}}{(2 \pi)^{d}}\left(\frac{T}{D}\right)^{\frac{d}{2}} D \zeta\left(\frac{d}{2}+1\right) \Gamma\left(\frac{d}{2}+2\right) \\
& \times \frac{1}{\cos \frac{\pi d}{4}}\left[\frac{d}{2}-\frac{1}{F_{0}}\left(1+F_{0}-\left(1+F_{0}\right)^{1-\frac{d}{2}}\right)\right] \\
\kappa^{\rho}-\kappa^{g}= & -\frac{1}{4} \frac{\Omega_{d}}{(2 \pi)^{d}}\left(\frac{T}{D}\right)^{\frac{d}{2}} D \zeta\left(\frac{d}{2}+1\right) \Gamma\left(\frac{d}{2}+2\right) \\
& \times \frac{1}{\cos \frac{\pi d}{4}}\left[1-\left(1+F_{0}\right)^{1-\frac{d}{2}}\right],
\end{aligned}
$$

where in the absence of the magnetic field $\sigma_{\mu \nu}=\sigma \delta_{\mu \nu}$ and the similar relation holds for the thermal conductivity. According to the previous discussion, for the Coulomb interaction the correct expressions are given by the limit $F_{0} \rightarrow+\infty$, with the exception of the term $\kappa^{\rho}-\kappa^{g}$ in $d=1$ for which, with logarithmic accuracy, the result is obtained by substituting [...] $\rightarrow$ $-a k \ln ^{1 / 2}\left(D k^{2} / T\right)$. The final answers for the corrections to the thermal conductivity are given in Eqs. (4.2)-(4.3).

We note that for $d=3$ the $\omega$ integration in $\hat{\sigma}$ and $\hat{\kappa}_{W F}$ is actually ultraviolet divergent. This divergence can be incorporated as a renormalization into the Drude results; this renormalization however does not invalidate the Wiedemann-Franz law.

\section{B. Two-dimensional system}

In order to evaluate the interaction corrections for the whole temperature range, we need the exact form of the 
propagators. In two dimensions they are:

$$
\begin{aligned}
& \mathcal{L}^{\alpha}\left(\omega, \boldsymbol{q} ; \boldsymbol{n}_{1}, \boldsymbol{n}_{2}\right)=\Omega_{d} \delta\left(\widehat{\boldsymbol{n}_{1} \boldsymbol{n}_{2}}\right) \mathcal{L}_{0}\left(\omega, \boldsymbol{q} ; \boldsymbol{n}_{1}\right) \\
& +\mathcal{L}_{0}\left(\omega, \boldsymbol{q} ; \boldsymbol{n}_{1}\right) \mathcal{L}_{0}\left(\omega, \boldsymbol{q} ; \boldsymbol{n}_{2}\right) \frac{\left(-i \omega \frac{F_{0}^{\alpha}}{1+F_{0}^{\alpha}}+\frac{1}{\tau}\right) \mathcal{C}}{\mathcal{C}-\left(-i \omega \frac{F_{0}^{\alpha}}{1+F_{0}^{\alpha}}+\frac{1}{\tau}\right)}
\end{aligned}
$$

where

$$
\begin{aligned}
& \mathcal{L}_{0}(\omega, \boldsymbol{q} ; \boldsymbol{n})=\frac{1}{-i \omega+i \boldsymbol{v} \cdot \boldsymbol{q}+1 / \tau} \\
& \mathcal{C}=\sqrt{(-i \omega+1 / \tau)^{2}+\left(v_{F} q\right)^{2}}
\end{aligned}
$$

Note that now the variables $\omega$ and $v_{F} q$ are limited only by the Fermi energy $E_{F}$.

As before, we need to find the non-equilibrium corrections $\delta N^{\rho, g}$ to the bosonic distribution functions. These are given again by Eqs. (6.16)-(6.17), but to obtain the thermal conductivity on the arbitrary temperature range, we calculate exactly (at linear order in $\nabla T$ ) the solution to Eq. (6.18):

$$
\begin{aligned}
\delta N^{1}=\tilde{N}\{ & \frac{\boldsymbol{q} \cdot \boldsymbol{\nabla} T}{v_{F} q^{2}}+\frac{i}{i v_{F} \boldsymbol{q} \cdot\left(\boldsymbol{n}_{1}-\boldsymbol{n}_{2}\right)+2 / \tau} \\
& \left.\times\left[\bar{\lambda}\left(\boldsymbol{n}_{1}\right) \boldsymbol{n}_{1}^{\perp}-\lambda\left(\boldsymbol{n}_{2}\right) \boldsymbol{n}_{2}^{\perp}\right] \cdot \nabla T\right\},
\end{aligned}
$$

where the bar indicates complex conjugation and we introduced the following quantities:

$$
\begin{aligned}
& \tilde{N}=v_{F} \tau \frac{\omega^{2}}{T} \frac{\partial N_{P}}{\partial \omega} \frac{F_{0}}{1+F_{0}}, \boldsymbol{n}^{\perp}=\boldsymbol{n}-\frac{(\boldsymbol{n} \cdot \boldsymbol{q}) \boldsymbol{q}}{q^{2}} \\
& \lambda(\boldsymbol{n})=\frac{a(\boldsymbol{n})}{a(\boldsymbol{n})-b}, \quad b=\frac{-i \omega F_{0}}{1+F_{0}}+\frac{1}{\tau} \\
& a(\boldsymbol{n})=\sqrt{\left(v_{F} q\right)^{2}+\left(-i \boldsymbol{q} \cdot \boldsymbol{v}+\frac{2}{\tau}\right)^{2}} .
\end{aligned}
$$

We will not calculate the corrections to the electrical conductivity, which would reproduce the results of Ref. 17, as shown in Appendix F [see also Ref. 18 for the generalization to the arbitrary disorder]. For convenience in the calculations, we separate in $\delta \hat{\kappa}_{i n}$ and $\hat{\kappa}^{\rho}$ the contributions due to $\delta N^{0}$ and $\delta N^{1}$ [cf. Eqs. (6.11c) and $(6.23)]$ :

$$
\begin{aligned}
\delta \hat{\kappa}_{i n}= & \delta \hat{\kappa}_{i n}^{0}+\delta \hat{\kappa}_{i n}^{1} \\
\delta \hat{\kappa}_{i n}^{0}= & \frac{2 \sigma_{D}}{e^{2} T} \int d \omega\left[\hat{\mathcal{S}}^{12}(\omega)-\hat{\mathcal{S}}^{11}(\omega)\right]\left[\frac{\omega^{3}}{4} \frac{\partial N_{P}}{\partial \omega}\right](6.35 \\
{\left[\delta \kappa_{i n}^{1}\right]_{\mu \nu}=} & v_{F} \int \frac{d \omega}{2 \pi} \omega \int \frac{d^{2} q}{(2 \pi)^{2}} \int \frac{d \boldsymbol{n}_{1} d \boldsymbol{n}_{2} d \boldsymbol{n}_{3}}{\left(\Omega_{2}\right)^{3}} \\
& n_{1 \mu} \operatorname{Re}\left\{\mathcal{L}_{12}^{\rho} \delta_{\nu} N_{23}^{1}+\mathcal{L}_{32}^{\rho} \delta_{\nu} N_{21}^{1}-\mathcal{L}_{12}^{\rho} \delta_{\nu} N_{21}^{1}\right\}
\end{aligned}
$$

where $k=2 \pi e^{2} \nu$ is the inverse screening radius. Next, we calculate the angular integrals in Eqs. (6.35c) and (6.36c) as well as the angular part of the momentum integrals

$$
\begin{aligned}
& \hat{\kappa}^{\rho}=\hat{\kappa}_{0}^{\rho}+\hat{\kappa}_{1}^{\rho} \\
& \hat{\kappa}_{0}^{\rho}-\hat{\kappa}^{g}=\frac{\sigma_{D}}{e^{2} T} \int d \omega \hat{\mathcal{B}}^{0}(\omega)\left[\frac{\omega^{3}}{4} \frac{\partial N_{P}}{\partial \omega}\right] \\
& \hat{\kappa}_{1}^{\rho}=-\int \frac{d \omega}{2 \pi} \omega \int \frac{d^{2} q}{(2 \pi)^{2}} \int \frac{d \boldsymbol{n}_{1} d \boldsymbol{n}_{2}}{\left(\Omega_{2}\right)^{2}}\left\{\hat{s}_{\mu} ; \mathcal{L}_{12}^{\rho} \delta_{\nu} N_{21}^{1}\right\}
\end{aligned}
$$

where, as in Eq. (6.13), we indicate with $\delta_{\nu} N^{1}$ the variational derivative with respect to the temperature gradient and

$$
\mathcal{B}_{\mu \nu}^{0}(\omega)=-\frac{4}{\pi \omega \nu} \int \frac{d^{2} q}{(2 \pi)^{2}} \int \frac{d \boldsymbol{n}_{1}}{\Omega_{2}} n_{1 \mu} n_{1 \nu} \operatorname{Re}\left[\mathcal{L}_{11}^{\rho}-\mathcal{L}_{11}^{g}\right]
$$

Expressions (6.8) for the electrical conductivity and (6.11b) for the elastic correction to the thermal conductivity and the definition (6.9) for the kernels remain unchanged. The momentum and angular integrals in the latter can be exactly calculated and for the singlet channel in the unitary limit we find:

$$
\begin{aligned}
\mathcal{E}_{\mu \nu}(\omega)= & -\frac{e^{2} \delta_{\mu \nu}}{\sigma_{D} 2 \pi^{2}} \frac{1}{2 \omega}\left[2-2 \omega \tau H(\omega \tau) \arctan \frac{1}{\omega \tau}\right. \\
& +\frac{(\omega \tau)^{2}}{2}\left(\frac{1}{2}-H(\omega \tau)\right) \ln \left(1+\frac{1}{(\omega \tau)^{2}}\right)(6.37 \mathrm{a}) \\
& \left.\quad-(\omega \tau)^{2} H(\omega \tau) \ln 2\right] \\
\mathcal{S}_{\mu \nu}^{11}(\omega)= & -\frac{e^{2} \delta_{\mu \nu}}{\sigma_{D} 2 \pi^{2}} \tau \frac{\pi}{2} \operatorname{sgn} \omega \\
\mathcal{S}_{\mu \nu}^{12}(\omega)= & -\frac{e^{2} \delta_{\mu \nu}}{\sigma_{D} 2 \pi^{2}} \tau\left\{(2 H(\omega \tau)-1) \arctan \frac{1}{\omega \tau} \quad(6.37 \mathrm{c})\right. \\
+ & \left.\frac{\pi}{2} \operatorname{sgn} \omega+\omega \tau H(\omega \tau)\left[\frac{1}{2} \ln \left(1+\frac{1}{(\omega \tau)^{2}}\right)+\ln 2\right]\right\}
\end{aligned}
$$

with the function $H$ defined as:

$$
H(x)=\frac{1}{4+x^{2}}
$$

To perform the momentum integral in Eq. (6.36d) we must keep the full form of the propagator in order to avoid the infrared divergence that we would obtain in the unitary limit:

$$
\begin{array}{r}
\mathcal{B}_{\mu \nu}^{0}(\omega)=-\frac{e^{2} \delta_{\mu \nu}}{\sigma_{D} 2 \pi^{2}} \frac{1}{\omega}\left\{\ln \left(\frac{D k^{2}}{|\omega|}\right)+\pi|\omega| \tau\right. \\
\left.-\frac{1}{2} \ln \left[1+(\omega \tau)^{2}\right]-\omega \tau \arctan \left(\frac{1}{\omega \tau}\right)\right\}
\end{array}
$$


and obtain:

$$
\begin{aligned}
& \int \frac{d \theta_{q}}{2 \pi} \int \frac{d \boldsymbol{n}_{1} d \boldsymbol{n}_{2} d \boldsymbol{n}_{3}}{\left(\Omega_{2}\right)^{3}} n_{1 \mu} \operatorname{Re}\left\{\mathcal{L}_{12}^{\rho} \delta_{\nu} N_{23}^{1}+\mathcal{L}_{32}^{\rho} \delta_{\nu} N_{21}^{1}\right\} \\
& =\frac{i}{4} \tilde{N} \delta_{\mu \nu}\left\{\frac{1}{(v q)^{2}} \frac{1}{\mathcal{C} \overline{\mathcal{C}}}\left[\frac{\mathcal{C} \bar{b}^{\prime}}{\mathcal{C}-b}+\frac{\overline{\mathcal{C}} b^{\prime}}{\overline{\mathcal{C}}-\bar{b}}\right]\left[\overline{\mathcal{C}}-\bar{b}^{\prime}-\mathcal{C}+b^{\prime}\right]\right. \\
& \left.+\frac{1}{(v q)^{2}}\left[\frac{\mathcal{C}-b^{\prime}}{\mathcal{C}}-\frac{\overline{\mathcal{C}}-\bar{b}^{\prime}}{\overline{\mathcal{C}}}\right]-\frac{1}{\mathcal{C} \overline{\mathcal{C}}}\left[\frac{\mathcal{C}}{\mathcal{C}-b}-\frac{\overline{\mathcal{C}}}{\overline{\mathcal{C}}-\bar{b}}\right]\right\} \\
& \int \frac{d \theta_{q}}{2 \pi} \int \frac{d \boldsymbol{n}_{1} d \boldsymbol{n}_{2}}{\left(\Omega_{2}\right)^{2}} n_{1 \mu} \operatorname{Re}\left\{\mathcal{L}_{12}^{\rho} \delta_{\nu} N_{21}^{1}\right\} \\
& =\frac{i}{4} \tilde{N} \delta_{\mu \nu} \frac{\tau}{2}\left\{[ \frac { \overline { \mathcal { C } } } { \overline { \mathcal { C } } - \overline { b } } - \frac { \mathcal { C } } { \mathcal { C } - b } ] \left[\frac{1}{\mathcal{C}}+\frac{1}{\overline{\mathcal{C}}]}\right.\right. \\
& -\frac{1}{(v q)^{2}}\left[\frac{\mathcal{C} \bar{b}^{\prime}}{\mathcal{C}-b}+\frac{\overline{\mathcal{C}} b^{\prime}}{\overline{\mathcal{C}}-\bar{b}}\right]\left[\frac{\mathcal{C}-b^{\prime}}{\mathcal{C}}-\frac{\overline{\mathcal{C}}-\bar{b}^{\prime}}{\overline{\mathcal{C}}}\right] .38 \mathrm{~b}
\end{aligned}
$$

The function $\mathcal{C}$ was defined in Eq. (6.32) and $b^{\prime}$ is given by the limit $F_{0} \rightarrow+\infty$ of $b$ in Eq. (6.34), where $\tilde{N}$ is also defined. The remaining integrals over the magnitude of the momentum can be evaluated approximately; the result can be written as:

$$
\delta \hat{\kappa}_{i n}^{1}+\hat{\kappa}_{1}^{\rho}=\frac{\sigma_{D}}{e^{2} T} \int d \omega \hat{\mathcal{B}}^{1}(\omega)\left[\frac{\omega^{3}}{4} \frac{\partial N_{P}}{\partial \omega}\right]
$$

with

$$
\begin{array}{r}
\mathcal{B}_{\mu \nu}^{1}(\omega)=\frac{e^{2} \delta_{\mu \nu}}{\sigma_{D} 2 \pi^{2}} \tau\left\{\frac{2 \omega \tau}{1+(\omega \tau)^{2}} \ln \left(\frac{v_{F} k}{2|\omega|}\right)\right. \\
\left.-\arctan \omega \tau-\omega \tau \ln \left(\frac{E_{F}}{\sqrt{\omega^{2}+\tau^{-2}}}\right) \cdot\right\}
\end{array}
$$

In the above kernel the first term in curly bracket originates from $\hat{\kappa}_{1}^{\rho}$ only - as discussed in Sec. VB no long range terms can be present in the electronic contribution to the thermal conductivity. Note that the second term in the above expression is beyond the logarithmic accuracy of our approximate calculation and must be dropped. Similarly, most of the terms in the other kernels can be neglected and collecting the logarithmic contributions:

$$
\begin{aligned}
\Delta \kappa_{s} & =-\frac{1}{2 \pi^{2} T} \int d \omega\left[\frac{\omega^{2}}{4} \frac{\partial N_{P}}{\partial \omega}\right] \\
& \times\left\{(\omega \tau)^{2} \ln \left(\frac{E_{F}}{|\omega|}\right)+\frac{2}{1+(\omega \tau)^{2}} \ln \left(\frac{v_{F} k}{|\omega|}\right)\right. \\
& \left.+\left[\frac{7}{12}(\omega \tau)^{2}-\frac{5}{6}+\frac{16}{3} \frac{1}{4+(\omega \tau)^{2}}\right] \ln \left(1+\frac{1}{(\omega \tau)^{2}}\right)\right\},
\end{aligned}
$$

where we defined the singlet and triplet channel corrections as:

$$
\left[\kappa-\kappa_{W F}\right]_{\mu \nu}=\left(\Delta \kappa_{s}+3 \Delta \kappa_{t}\right) \delta_{\mu \nu}
$$

The final integration can now be performed within the logarithmic accuracy; we find [cf. Eq. (4.4b)]:

$$
\begin{aligned}
& \Delta \kappa_{s}=-\frac{\pi^{2}}{15} T(T \tau)^{2} \ln \left(\frac{E_{F}}{T}\right) \\
& +\frac{T}{6} g_{1}(2 \pi T \tau) \ln \left(\frac{v_{F} k}{T}\right)-\frac{T}{24} g_{2}(\pi T \tau) \ln \left(1+\frac{1}{(T \tau)^{2}}\right)
\end{aligned}
$$

where

$$
\begin{aligned}
& g_{1}(x)=\frac{3}{x^{2}}\left\{\frac{1}{x}\left[2 \psi^{\prime}\left(\frac{1}{x}\right)-x^{2}\right]-2\right\} \\
& g_{2}(x)=\frac{14}{15} x^{2}+\frac{8}{3} g_{1}(x)-\frac{5}{3},
\end{aligned}
$$

and $\psi^{\prime}$ is the derivative of the digamma function. Since the asymptotic behaviour of $g_{1}(x)$ is

$$
g_{1}(x)=\left\{\begin{array}{c}
1-\frac{1}{5} x^{2}+\frac{1}{7} x^{4}+\ldots x \ll 1 \\
\frac{3}{x}-\frac{6}{x^{2}}+\frac{\pi^{2}}{x^{3}}+\ldots x \gg 1
\end{array},\right.
$$

both these functions tend to 1 as $T \tau \rightarrow 0$; therefore in the diffusive limit the main contribution is $T \ln \left(D k^{2} / T\right) / 12$. On the other hand, for $T \tau \gg 1$ the first term in Eq. (6.41) is the dominant one.

Turning to the triplet channel, for simplicity we will restrict ourselves to the limiting diffusive and quasiballistic cases, although one can extend the calculation to the whole temperature range, as done in Ref. 17 for the electrical conductivity.

In the diffusive limit $T \tau \ll 1$ we know from our previous analysis that we can discard the $\mathcal{B}^{1}$ term as well as the $\mathcal{S}$ terms. The relevant kernels are then ${ }^{44}$ :

$$
\begin{gathered}
\mathcal{E}_{\mu \nu}(\omega)=-\frac{e^{2} \delta_{\mu \nu}}{\sigma_{D} 2 \pi^{2} \omega}\left[1-\frac{1}{F_{0}^{\sigma}} \ln \left(1+F_{0}^{\sigma}\right)\right] \\
\mathcal{B}_{\mu \nu}^{0}(\omega)=-\frac{e^{2} \delta_{\mu \nu}}{\sigma_{D} 2 \pi^{2} \omega} \ln \left(1+F_{0}^{\sigma}\right)
\end{gathered}
$$

which substituted into Eqs. (6.11)-(6.36b) give

$$
\Delta \kappa_{t}=-\frac{T}{18}\left[1-\frac{1}{F_{0}^{\sigma}} \ln \left(1+F_{0}^{\sigma}\right)\right]+\frac{T}{12} \ln \left(1+F_{0}^{\sigma}\right) .
$$

In the opposite limit $T \tau \gg 1$, the main contribution comes, as for the singlet channel, from the logarithmic divergence at large momentum in the kernel $\mathcal{B}^{1}$ :

$\mathcal{B}_{\mu \nu}^{1}(\omega)=-\frac{e^{2} \delta_{\mu \nu}}{\sigma_{D} 2 \pi^{2}} \tau\left\{\omega \tau \ln \left(\frac{E_{F}}{\sqrt{\omega^{2}+\tau^{-2}}}\right)\right\}\left(\frac{F_{0}^{\sigma}}{1+F_{0}^{\sigma}}\right)^{2}$.

Then, the correction to the thermal conductivity is:

$$
\Delta \kappa_{t}=-\frac{\pi^{2}}{15} T(T \tau)^{2} \ln \left(\frac{E_{F}}{T}\right)\left(\frac{F_{0}^{\sigma}}{1+F_{0}^{\sigma}}\right)^{2}
$$

which concludes the derivation of Eq. (4.4c). 
This correction to the thermal conductivity (and the corresponding one in the singlet channel) is the inelastic processes contribution to the energy relaxation rate ${ }^{45}$. In the clean system, such inelastic processes cannot relax momentum (because of Galilean invariance) and hence they do not affect the electrical conductivity, but they can contribute to the energy relaxation rate $\Gamma_{\epsilon}$. In the kinetic theory, the thermal conductivity can be written, up to a numerical coefficient, as:

$$
\kappa \propto T E_{F} / \Gamma_{\epsilon}
$$

and the rate is given by the sum of the rates for the relevant processes, namely the electron-impurity and electron-electron scattering rates:

$$
\Gamma_{\epsilon}=\Gamma_{i m p}+\Gamma_{e-e}
$$

with $\Gamma_{i m p}=1 / \tau$ and

$$
\Gamma_{e-e}=a \frac{T^{2}}{E_{F}} \ln \left(\frac{E_{F}}{T}\right)
$$

Here $a$ is a constant whose exact value is irrelevant for our argument. In the limit $\left(T^{2} / E_{F}\right) \ln \left(E_{F} / T\right) \ll 1 / \tau$ we can expand the expression for the total rate, substitute the result into the above formula for $\kappa$ and obtain:

$$
\kappa \propto T \tau E_{F}-a T(T \tau)^{2} \ln \left(\frac{E_{F}}{T}\right)
$$

The first term on the right hand side is the usual Drude result for the thermal conductivity and the second term has the form of the correction (6.47). Note that in the opposite limit (clean system) one would find:

$$
\kappa \propto \frac{E_{F}^{2}}{T \ln \left(\frac{E_{F}}{T}\right)}
$$

in agreement with the result of Ref. 29 .

\section{Specific heat}

Recalling our discussion on the structure of the kinetic equation in Section II, we write the total specific heat as the sum of the electronic and bosonic contributions:

$$
\begin{aligned}
C_{V} & =C_{V}^{0}+\delta C_{V}, \\
C_{V}^{0} & =\frac{\partial u_{e}}{\partial T}=\frac{\pi^{2}}{3} \nu T, \\
\delta C_{V} & =\frac{\partial}{\partial T}\left(u_{\rho}-u_{g}\right),
\end{aligned}
$$

where, according to Eq. (5.93c), the bosonic energy densities [in the equilibrium (3.1)] are

$$
u_{\alpha}=\int d \omega \omega b^{\alpha}(\omega) N_{P}(\omega)
$$

with $^{46}$

$$
b^{\alpha}(\omega)=\frac{\operatorname{Re}}{2 \pi} \int \frac{d^{d} q}{(2 \pi)^{d}} \operatorname{Tr}_{\boldsymbol{n}}\left\{\frac{1}{1+\hat{F}^{\alpha}} ; \mathcal{L}^{\alpha}(\omega, \boldsymbol{q})\right\} .
$$

As before, we will consider explicitly the singlet channel, short range interaction in the zeroth harmonic approximation for the Fermi liquid constant, which we denote with $F_{0}$. The results for the long range interaction in the unitary limit are obtained by letting $F_{0}^{\rho} \rightarrow+\infty$. For the triplet channel one must substitute $F_{0}^{\rho}$ with $F_{0}^{\sigma}$ and multiply by an overall factor of 3 . The final answer with the correct coefficients is given in Sec. IV B.

In the diffusive limit, to which we restrict our attention for $d=1,3$, we have:

$b^{\rho}(\omega)-b^{g}(\omega)=\frac{\operatorname{Re}}{2 \pi} \int \frac{d^{d} q}{(2 \pi)^{d}}\left[\frac{1}{1+F_{0}^{\rho}} L_{0}^{\rho}(\omega, \boldsymbol{q})-L_{0}^{g}(\omega, \boldsymbol{q})\right]$

and the functions $L_{0}^{\alpha}$ were defined in Eqs. (6.20) and (6.21). After the integration over momentum we find:

$$
\begin{aligned}
b^{\rho}(\omega)-b^{g}(\omega) & =\frac{\Omega_{d}}{(2 \pi)^{d}}\left(\frac{|\omega|}{D}\right)^{\frac{d}{2}} \frac{1}{4 \omega} \\
\times & \frac{\cos \frac{\pi}{4}(d-2)}{\sin \frac{\pi}{2}(d-2)}\left[1-\frac{1}{\left(1+F_{0}^{\rho}\right)^{\frac{d}{2}}}\right]
\end{aligned}
$$

which inserted into Eq. (6.48c) gives:

$$
\begin{aligned}
\delta C_{V}= & \frac{1}{2} \frac{\Omega_{d}}{(2 \pi)^{d}}\left(\frac{T}{D}\right)^{\frac{d}{2}} \zeta\left(\frac{d}{2}+1\right) \Gamma\left(\frac{d}{2}+2\right) \\
& \times \frac{\cos \frac{\pi}{4}(d-2)}{\sin \frac{\pi}{2}(d-2)}\left[1-\frac{1}{\left(1+F_{0}^{\rho}\right)^{\frac{d}{2}}}\right] .
\end{aligned}
$$

The relevant numerical values for the zeta and gamma functions are given after Eq. (6.27), which has been used to perform the $\omega$ integral.

For $d=2$ we can keep the full form of the propagators to find the singular contribution to the specific heat at arbitrary value of $T \tau$ :

$$
\begin{array}{r}
b^{\rho}(\omega)-b^{g}(\omega)=-\frac{\operatorname{Re}}{2 \pi} \int \frac{d^{2} q}{(2 \pi)^{2}}\left[\frac{F_{0}^{\rho}}{1+F_{0}^{\rho}} \frac{1}{\mathcal{C}-b}\right. \\
\left.-\frac{(-i \omega+1 / \tau)}{\mathcal{C}}\left(\frac{1}{\mathcal{C}-b}-\frac{1}{\mathcal{C}-1 / \tau}\right)\right],
\end{array}
$$

with $\mathcal{C}$ and $b$ defined respectively in Eqs. (6.32) and (6.34). The first term in the integral is formally divergent at $|q| \rightarrow \infty$ and this divergence gives a linear in $T$ contribution to the specific heat which does not depend on disorder. This term must be disregarded as all the linear terms are included in the definition of the effective electron mass - taking it into account here would be a double counting. To regularize the integral, we replace $\frac{1}{\mathcal{C}-b} \rightarrow \frac{1}{\mathcal{C}-b}-\frac{1}{\mathcal{C}}$ in the first line. 
Performing the momentum integral we obtain:

$$
\begin{aligned}
b^{\rho}(\omega) & -b^{g}(\omega)=-\frac{1}{8 \pi^{2} D}\left[\frac{F_{0}^{\rho}}{1+F_{0}^{\rho}} \ln \left(\frac{E_{F}}{|\omega|}\right)\right. \\
& \left.+\left(\frac{F_{0}^{\rho}}{1+F_{0}^{\rho}}\right)^{2} \frac{\pi}{2} \tau|\omega|-\frac{1}{1+F_{0}^{\rho}} \ln \left(1+F_{0}^{\rho}\right)\right] .
\end{aligned}
$$

The final answer for the correction to the specific heat is then:

$$
\begin{aligned}
\delta C_{V}= & -\frac{1}{12} \frac{T}{D} \frac{1}{1+F_{0}^{\rho}}\left[F_{0}^{\rho} \ln \left(\frac{E_{F}}{T}\right)-\ln \left(1+F_{0}^{\rho}\right)\right] \\
& -\frac{1}{4 \pi^{2}}\left[(2 \gamma-3) \zeta(2)-2 \zeta^{\prime}(2)\right] \frac{T}{D} \frac{F_{0}^{\rho}}{1+F_{0}^{\rho}} \\
& -\frac{3}{4 \pi} \zeta(3) \frac{T}{D}(T \tau)\left(\frac{F_{0}^{\rho}}{1+F_{0}^{\rho}}\right)^{2}
\end{aligned}
$$

where $\zeta(2) \approx 1.645, \zeta^{\prime}(2) \approx-0.938$ and $\zeta(3) \approx 1.202$. In the quasi ballistic limit $\tau \rightarrow+\infty$ only the last line is relevant:

$$
\delta C_{V}=-\frac{3}{2 \pi} \zeta(3)\left(\frac{F_{0}^{\rho}}{1+F_{0}^{\rho}}\right)^{2} \frac{T^{2}}{v_{F}^{2}}
$$

This $T^{2}$ correction to the specific heat has the same form found for two-dimensional Fermi liquids ${ }^{28}$.

As discussed before, the long-range interaction can be accounted for by taking the limit $F_{0}^{\rho} \rightarrow+\infty$, while the triplet channel contribution is three times larger - see also Sec. IV B.

\section{CONCLUSIONS}

Locality on the scale determined by the temperature and the validity of the conservation laws are two main requirements for a proper kinetic description of any system. In the present paper we derived such a description for the interaction effects in disordered metals [assuming that the clean counterpart of the system is a stable Fermi liquid].

We showed that this description requires the introduction, along with the usual fermionic quasiparticle distribution function, of additional bosonic distribution functions. These neutral bosons are of two types: (i) the ones describing the oscillation in charge density (singlet) or spin density (triplet) and (ii) fictitious (ghost) bosons which prevent over-counting the degrees of freedoms (electron-hole pairs) already included in the fermionic part. The obtained conservation laws together with gauge invariance allowed for the unambiguous definition of the corresponding electric and energy currents.

For the electric transport the neutral bosons are not important and our description reproduces the known results for the correction to the conductivity obtained in
Ref. 11 for the diffusive regime and in Ref. 17 in the ballistic and crossover regime.

The neutral bosons, however, are crucial for the thermal properties of the system. Namely, their contributions to the energy density are responsible for the nonanalytic corrections to the specific heat, see Eqs. (4.6) and (4.8). Our kinetic equation approach reproduces the results for the interaction corrections to the specific heat previously calculated within the equilibrium diagrammatic technique ${ }^{12}$. Moreover the neutral bosons contributions to the energy current cause the violation of the Wiedemann-Franz law, see Eq. (4.1) and the discussion that follows it. The violation is stronger for low dimensionality systems $(d=1,2)$ in the diffusive regime, see Eqs. (4.2) and (4.4b). Other effects contributing to the violation of the Wiedemann-Franz law are the energy dependence of the electron elastic scattering and the inelastic scattering of the electrons on the neutral bosons. The latter effect was found to be relevant in the quasiballistic regime $T \tau \gg \hbar$ for two-dimensional systems, see Eqs. (4.4).

The violation of the Wiedemann-Franz law was investigated before in the diffusive regime in Refs. 20 and 23 within the "quantum kinetic equation" approach and by Kubo formula in Ref. 22. Ironically, even though the forms of the energy current operator used in those references are wrong, the final results for the thermal conductivity are consistent with our Eqs. (4.2)-(4.4). We think that this agreement is accidental.

\section{Acknowledgments}

We are grateful to B.L. Altshuler for initiating this work. Interesting conversations with M.Yu. Reizer are acknowledged. We would like to thank A.I. Larkin, A.J. Millis and B.N. Narozhny for discussions of our results. I.A. was supported by Packard foundation.

\section{APPENDIX A: ON THE CORRECTION TO THE THERMODYNAMIC POTENTIAL}

Standard analytic continuation of Eq. (2.17) gives:

$$
\begin{aligned}
\delta \Omega & =\int \frac{d \omega}{4 \pi} \operatorname{coth}\left(\frac{\omega}{2 T}\right) \int \frac{d^{d} q}{(2 \pi)^{d}} \operatorname{Im} \ln \left(1+\frac{F}{\nu} \Pi^{R}(\omega, \boldsymbol{q})\right) \\
& =\int \frac{d \omega}{2 \pi} \frac{1}{2} \operatorname{coth}\left(\frac{\omega}{2 T}\right) \operatorname{Im} \operatorname{Tr} \ln \left(1+\frac{\hat{F}}{\nu} \hat{\Pi}^{R}\right)
\end{aligned}
$$

In the second line we used the operator notation - see Eqs. (3.3) and (3.13) - which gives the correct generalization for the momentum dependent Fermi liquid parameter. Substituting the transform of the explicit expression (5.43a) for the polarization operator, the argument of the 
logarithm can be rewritten as:

$$
(1+\hat{F})\left[\left(\hat{\mathcal{L}}^{g}\right)^{-1}+\frac{\hat{F}}{1+\hat{F}} i \omega\right] \hat{\mathcal{L}}^{g}
$$

According to the definition (3.11), the term in square brackets is $\left(\hat{\mathcal{L}}^{\rho}\right)^{-1}$. Using the property

$$
\operatorname{Tr} \ln (\hat{A} \hat{B})=\operatorname{Tr} \ln \hat{A}+\operatorname{Tr} \ln \hat{B}
$$

and since $\ln (1+\hat{F})$ does not contribute to the imaginary part, we conclude that

$$
\operatorname{Im} \operatorname{Tr} \ln \left(1+\frac{\hat{F}}{\nu} \hat{\Pi}^{R}\right)=-\operatorname{Im} \operatorname{Tr}\left[\ln \hat{\mathcal{L}}^{\rho}-\ln \hat{\mathcal{L}}^{g}\right]
$$

Substituting this identity into Eq. (A1) we finally obtain Eq. (2.18).

\section{APPENDIX B: ON THE MICROSCOPIC FORM OF THE ENERGY CURRENT OPERATOR}

The action entering into the partition function that describes the electron gas in the presence of an external electric field is

$$
S=\int d t d^{d} r\left[i \psi^{\dagger} \partial_{t} \psi-\psi^{\dagger} \hat{H}_{g i} \psi-\psi^{\dagger} \varphi \psi\right]
$$

with the condition: $\boldsymbol{\nabla} \times \mathbf{A}=0$, which ensures the absence of the magnetic field. The variables $(t, \boldsymbol{r})$ on which all the fields depend have been suppressed. The gauge invariant part of the Hamiltonian for the non-interacting system has the usual form:

$$
\begin{aligned}
\psi^{\dagger} \hat{H}_{g i} \psi= & \frac{1}{2 m}(i \boldsymbol{\nabla}+\mathbf{A}) \psi^{\dagger}(-i \boldsymbol{\nabla}+\mathbf{A}) \psi \\
& +\psi^{\dagger} V_{i m p} \psi
\end{aligned}
$$

where $V_{i m p}$ is the impurity potential and the potentials $\varphi$ and $\mathbf{A}$ describe the external electric field:

$$
e \boldsymbol{E}_{\text {ext }}=-\boldsymbol{\nabla} \varphi+\partial_{t} \mathbf{A}
$$

As usual, the charge conservation law

$$
\partial_{t} \rho+\nabla \cdot \boldsymbol{j}=0
$$

follows from the requirement of gauge invariance, with the charge and current densities given by:

$$
\begin{aligned}
& \rho=e \psi^{\dagger} \psi \\
& \boldsymbol{j}=\frac{e}{2 m}\left[\psi^{\dagger}(-i \boldsymbol{\nabla}+\mathbf{A}) \psi+(i \boldsymbol{\nabla}+\mathbf{A}) \psi^{\dagger} \psi\right]
\end{aligned}
$$

The invariance of the action upon the replacement

$$
\psi(t, \boldsymbol{r}) \rightarrow \psi(t+\alpha(t, \boldsymbol{r}), \boldsymbol{r})
$$

[and the similar replacement for $\psi^{\dagger}$ ] is the basis for the derivation of the energy conservation law. A straightforward calculation gives

$$
\begin{aligned}
& \frac{\delta S}{\delta \alpha}=0=\partial_{t}^{\prime}\left(\psi^{\dagger} \hat{H}_{g i} \psi\right)+\varphi \partial_{t}\left(\psi^{\dagger} \psi\right) \\
& -\frac{i}{2 m} \boldsymbol{\nabla} \cdot\left[\partial_{t} \psi^{\dagger}(-i \boldsymbol{\nabla}+\mathbf{A}) \psi-(i \boldsymbol{\nabla}+\mathbf{A}) \psi^{\dagger} \partial_{t} \psi\right]
\end{aligned}
$$

where the prime means that the derivative acts on $\psi, \psi^{\dagger}$ only. By adding and subtracting terms proportional to $\varphi$ in the last bracket and to $\partial_{t} A$ in the first term, we find the energy conservation law:

$$
\partial_{t} u_{0}+\nabla \cdot \boldsymbol{j}_{\epsilon}^{0}=\boldsymbol{j} \cdot \boldsymbol{E}_{\text {ext }}-\frac{1}{e} \varphi\left[\partial_{t} \rho+\nabla \cdot \boldsymbol{j}\right]
$$

where

$$
\begin{aligned}
& u_{0}=\psi^{\dagger} \hat{H}_{g i} \psi \\
& \boldsymbol{j}_{\epsilon}^{0}=-\frac{1}{2 m}\left[\left(i \partial_{t}+\varphi\right) \psi^{\dagger}(-i \boldsymbol{\nabla}+\mathbf{A}) \psi\right. \\
&\left.\quad-(i \boldsymbol{\nabla}+\mathbf{A}) \psi^{\dagger}\left(i \partial_{t}-\varphi\right) \psi\right]
\end{aligned}
$$

The first term on the right hand side of Eq. (B6) is the usual Joule heat; the last term in that equation is not gauge invariant, but it vanishes because it is proportional to the continuity equation (B3).

We now consider the generalization to the interacting case. The Hamiltonian now contain an additional term:

$$
\frac{1}{2} \int d^{d} r_{1} \psi^{\dagger} \psi(\boldsymbol{r}) V\left(\boldsymbol{r}-\boldsymbol{r}_{1}\right) \psi^{\dagger} \psi\left(\boldsymbol{r}_{1}\right)
$$

where $V(\boldsymbol{r})=e^{2} /|\boldsymbol{r}|$ describes the density-density Coulomb interaction, which can be decoupled by the Hubbard-Stratonovich transformation. This amount to the introduction of the quantum fields $\phi$ and $\mathcal{A}$ in the action by adding the term

$$
-\psi^{\dagger} \phi \psi+\frac{1}{2} \boldsymbol{E}_{f l}^{2}, \quad e \boldsymbol{E}_{f l}=-\nabla \phi+\partial_{t} \mathcal{A}
$$

and redefining the vector potential as the sum of the external and fluctuating ones:

$$
\mathbf{A} \rightarrow \mathbf{A}_{e x t}+\mathcal{A}
$$

The variation of the action with respect to the fluctuating potentials results in the first and fourth Maxwell equations relating the fluctuating electric field to the charge and current densities:

$$
\boldsymbol{\nabla} \cdot \boldsymbol{E}_{f l}=\rho, \quad 0=\boldsymbol{j}+\partial_{t} \boldsymbol{E}_{f l}
$$

where the electric current is defined in Eq. (B4), but the substitution (B9) must be performed.

To obtain the energy conservation law we must consider the further transformation

$$
\phi(t, \boldsymbol{r}) \rightarrow \phi(t+\alpha(t, \boldsymbol{r}), \boldsymbol{r})
$$


and the similar one for $\mathcal{A}$. Proceeding as before we find the conservation law:

$$
\begin{aligned}
& \frac{\partial u^{?}}{\partial t}+\nabla \cdot \boldsymbol{j}_{\epsilon}^{?}=\boldsymbol{j} \cdot \boldsymbol{E}_{e x t} \\
& u^{?}=u_{0}-\frac{1}{2} \boldsymbol{E}_{f l}^{2}+\frac{1}{e}\left[\rho \phi+\frac{\partial \mathcal{A}}{\partial t} \cdot \boldsymbol{E}_{f l}\right] \\
& \boldsymbol{j}_{\epsilon}^{?}=\boldsymbol{j}_{\epsilon}^{0}-\frac{1}{e} \phi \boldsymbol{j}+\frac{1}{e}\left[\phi \boldsymbol{j}-\frac{\partial \phi}{\partial t} \boldsymbol{E}_{f l}\right]
\end{aligned}
$$

where $u_{0}, \boldsymbol{j}_{\epsilon}^{0}$ are defined in Eq. (B7) [with the substitution (B9)]. Given the form of Eq. (B11), one might be tempted to call $u^{?}$ and $\boldsymbol{j}_{\epsilon}^{?}$ the energy and energy current densities so that the conservation law takes exactly the same form as in the non-interacting case. However, such a redefinition would result in gauge dependent expressions for the densities, since the terms the square brackets taken separately are not gauge invariant. Hence this naive redefinition of the conserved quantities is unphysical, since any physical perturbation can be coupled only to gauge invariant quantities. To find the gauge invariant definitions, we rewrite the contribution of those non-gauge invariant terms as:

$$
\begin{aligned}
& \frac{\partial}{\partial t}\left[\rho \phi+\frac{\partial \mathcal{A}}{\partial t} \cdot \boldsymbol{E}_{f l}\right]+\boldsymbol{\nabla}\left[\phi \boldsymbol{j}-\frac{\partial \phi}{\partial t} \boldsymbol{E}_{f l}\right] \\
& =\frac{1}{e} \phi\left[\partial_{t} \rho+\boldsymbol{\nabla} \cdot \boldsymbol{j}\right]+\frac{1}{e} \frac{\partial \phi}{\partial t}\left[\rho-\boldsymbol{\nabla} \cdot \boldsymbol{E}_{f l}\right] \\
& +\frac{1}{e} \frac{\partial}{\partial t}\left(\partial_{t} \mathcal{A}-\boldsymbol{\nabla} \phi\right) \cdot \boldsymbol{E}_{f l}+\frac{1}{e}\left[\boldsymbol{\nabla} \phi \cdot \boldsymbol{j}+\partial_{t} \mathcal{A} \partial_{t} \boldsymbol{E}_{f l}\right]
\end{aligned}
$$

Here the first line vanishes because of charge conservation, Eq. (B3), and because of the first of the Maxwell equations (B10). As for the second line, we use the second Maxwell equation to eliminate the current; in the result we substitute the definition for the fluctuating field given in Eq. (B8) and we obtain that the second line of Eq. (B11) equals $\partial_{t} \boldsymbol{E}_{f l}^{2}$. This enables us to conclude that the correct, gauge invariant expressions for the energy and energy current densities are:

$$
\begin{aligned}
u= & \psi^{\dagger} \hat{H}_{g i} \psi+\frac{1}{2} \boldsymbol{E}_{f l}^{2} \\
\boldsymbol{j}_{\epsilon}=- & \frac{1}{2 m}\left[\left(i \partial_{t}+\varphi\right) \psi^{\dagger}(-i \boldsymbol{\nabla}+\mathbf{A}) \psi\right. \\
& \left.\quad-(i \boldsymbol{\nabla}+\mathbf{A}) \psi^{\dagger}\left(i \partial_{t}-\varphi\right) \psi\right]
\end{aligned}
$$

where the potentials are the total ones:

$$
\mathbf{A}=\mathbf{A}_{e x t}+\mathcal{A}, \quad \varphi=\varphi_{e x t}+\phi
$$

We note that these expressions are gauge invariant with respect to gauge transformations of both the external and fluctuating potentials. We believe, that only such quantities can be coupled to the "gravitational field" in the Luttinger scheme for the calculation of the thermal conductivity $^{5}$.

The same final answer is obtained if the interaction is decoupled in the "gauge-fixed" form $\boldsymbol{A}=0$. In this case,

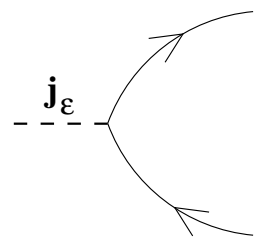

(a)

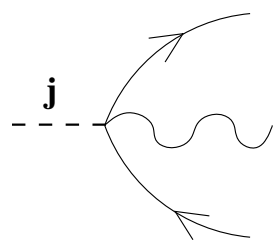

(b)
FIG. 4: (a) the energy current vertex for the non-interacting case; (b) the additional vertex induced by the interaction. The solid lines with arrows are electron Green's functions, the wavy line is the interaction propagator, the dashed lines are the "standard" (non-interacting case) energy and electric current operators defined respectively in Eqs. (B7b) and (B4).

which is the most widely used in the literature, there are two contributions to the energy current vertex in the diagrammatic approach, see Fig. 4: beside the usual vertex of the non-interacting case, which arises from the terms $\partial_{\tau} \psi^{\dagger} \boldsymbol{\nabla} \psi$, there is an additional vertex from the $\phi \psi^{\dagger} \nabla \psi$ terms. These vertices were not taken into account in Refs. 20,22,23. However, analogous vertices were previuosly considered in the calculations of the thermoelectric coefficient with the inclusion of the electronelectron interaction in the particle-hole channel ${ }^{30,34}$ and in the Cooper channel ${ }^{31}$ and for the electron-phonon interaction $^{32}$.

\section{APPENDIX C: ALTERNATIVE PARAMETRIZATION}

The operator $\hat{H}_{\mathrm{e}-\mathrm{h}}$ defined in Eq. (5.54) is clearly not a standard Hamiltonian. However, one can introduce a different definition for the propagator $\mathcal{L}^{\rho}$

$$
\left[\frac{\partial}{\partial t_{1}}+i \hat{H}_{\mathrm{e}-\mathrm{h}}\left(-i \boldsymbol{\nabla}_{1}\right)-\widehat{\mathrm{St}}_{s}^{\text {out }}\right] \mathcal{L}^{\rho}=\delta_{12}
$$

such that the (new) $\hat{H}_{\text {e-h }}$ operator is indeed a Hamiltonian:

$$
\begin{gathered}
\hat{H}_{\mathrm{e}-\mathrm{h}}(\boldsymbol{q}) \equiv \hat{\mathcal{F}} \boldsymbol{v} \cdot \boldsymbol{q} \hat{\mathcal{F}}, \quad{\widehat{\mathrm{St}^{\text {out }}}}_{\text {out }} \equiv \hat{\mathcal{F}} \widehat{\mathrm{St}_{\tau}} \hat{\mathcal{F}}, \\
\hat{\mathcal{F}} \equiv(1+\hat{F})^{1 / 2}
\end{gathered}
$$

[the action of the operator $\hat{F}$ was defined in Eq. (5.49)]. Proceeding as in Section VD, one obtains the following expressions for the $\mathcal{K}$ propagators:

$$
\begin{gathered}
\nu \hat{\mathcal{K}}^{R}=\left(\partial_{t}\right)^{-1}\left[\hat{\mathcal{L}}^{g}-\hat{\mathcal{F}} \hat{\mathcal{L}}^{\rho} \hat{\mathcal{F}}\right] \\
\nu \hat{\mathcal{K}}^{A}=-\left[\hat{\overline{\mathcal{L}}}^{g}-\hat{\mathcal{F}} \hat{\overline{\mathcal{L}}}^{\rho} \hat{\mathcal{F}}\right]\left(\partial_{t}\right)^{-1} \\
\nu \hat{\mathcal{K}}^{K}=-i\left(\partial_{t}\right)^{-1}\left[\hat{\mathcal{L}}^{g} \hat{\mathcal{N}}^{g}+\hat{\mathcal{N}}^{g} \hat{\overline{\mathcal{L}}}^{g}\right]\left(\partial_{t}\right)^{-1} \\
+i\left(\partial_{t}\right)^{-1} \hat{\mathcal{F}}\left[\hat{\mathcal{L}}^{\rho} \hat{\mathcal{N}}^{\rho}+\hat{\mathcal{N}}^{\rho} \overline{\hat{\mathcal{L}}}^{\rho}\right] \hat{\mathcal{F}}\left(\partial_{t}\right)^{-1} .
\end{gathered}
$$


The "kinetic equation" for $\mathcal{N}^{g}$, Eq. (5.64a), remains unchanged, while $\mathcal{N}^{\rho}$ now obeys:

$$
\left[\partial_{t}+\hat{H}_{\mathrm{e}-\mathrm{h}}(-i \boldsymbol{\nabla}) ; \hat{\mathcal{N}}^{\rho}\right]=\widehat{\mathrm{St}}^{\rho}\left\{\mathcal{N}^{\rho}, g^{K}\right\}
$$

with

$$
\widehat{\mathrm{St}}^{\rho}\left\{\mathcal{N}^{\rho}, g^{K}\right\} \equiv 2\left\{{\widehat{\mathrm{St}_{s}}}^{\text {out }} ; \hat{\mathcal{N}}^{\rho}\right\}+2 \hat{\mathcal{F}} \hat{M} \hat{\mathcal{F}}
$$

or, after the Wigner transforms (5.61)-(5.63):

$$
\begin{aligned}
& \omega\left[\partial_{t} \hat{N}^{\rho}+\left\{\hat{\boldsymbol{s}}, \boldsymbol{\nabla} \hat{N}^{\rho}\right\}+i\left[\hat{H}_{\mathrm{e}-\mathrm{h}}(\boldsymbol{q}), \hat{N}^{\rho}\right]\right]=\widehat{\mathrm{St}}^{\rho}\left\{N^{\rho}, f\right\} \\
& \widehat{\mathrm{St}}^{\rho}\left\{N^{\rho}, f\right\}\left(\omega, \boldsymbol{n}_{1}, \boldsymbol{n}_{2}\right)=\int d \epsilon \int \frac{d \boldsymbol{n}_{3} \ldots d \boldsymbol{n}_{6}}{\Omega_{d}^{4}} \\
& {\left[\mathcal{F}_{16} \gamma_{34}^{6} \Upsilon_{52 ; 43}^{\rho}(\epsilon, \omega) \mathcal{F}_{45}+\mathcal{F}_{56} \gamma_{34}^{6} \Upsilon_{15 ; 34}^{\rho}(\epsilon, \omega) \mathcal{F}_{42}\right]}
\end{aligned}
$$

with the definitions (5.27) and (5.66) for $\gamma$ and $\Upsilon$.

We can then proceed as in Sec. VF and obtain the conservation laws (5.89) and (5.94); the only formal difference is in the definition of the bosonic energy density, which now is:

$$
u_{\alpha}(t, \boldsymbol{r})=\frac{1}{2} \operatorname{Tr}_{\boldsymbol{n}}\left[\hat{\mathcal{L}}^{\alpha} \hat{\mathcal{N}}^{\alpha}\right]
$$

In the alternative parametrization the formalism can be developed with no more difficulties than in the original one. However the evaluation of the thermal conductivity become cumbersome. Also in the original parametrization it is easier to include (at least perturbatively) the effects due to higher harmonics of the Fermi liquid parameters.

\section{APPENDIX D: DERIVATION OF THE ELECTRONS COLLISION INTEGRAL}

The calculation of the matrix collision integral is simplified by the introduction of two functions $A(t, \boldsymbol{r}, \boldsymbol{n}, \tilde{\boldsymbol{n}})$ and $B(t, \boldsymbol{r}, \boldsymbol{n}, \tilde{\boldsymbol{n}})$ such that

$$
e^{i \hat{K}(t, \boldsymbol{n}, \boldsymbol{r})} e^{-i \hat{K}(t, \tilde{\boldsymbol{n}}, \boldsymbol{r})}=\left(A \hat{\mathbb{1}}_{K}+B \hat{\sigma}_{K}^{x}\right)_{\boldsymbol{n}, \tilde{\boldsymbol{n}}}
$$

We remind that $\hat{K}=K_{+} \hat{\mathbb{1}}_{K}+K_{-} \hat{\sigma}_{K}^{x}$ and

$$
\hat{\sigma}_{K}^{x}=\left(\begin{array}{ll}
0 & 1 \\
1 & 0
\end{array}\right)
$$

The collision integral - right hand side of Eq. (5.22) in the matrix notation is then:

$$
\begin{aligned}
& \frac{1}{2 \tau}\left\langle\left[\hat{g}(\boldsymbol{n}) \circ\left(A \hat{\mathbb{1}}_{K}+B \hat{\sigma}_{K}^{x}\right)_{\boldsymbol{n}, \tilde{\boldsymbol{n}}} \hat{g}(\tilde{\boldsymbol{n}})\left(A \hat{\mathbb{1}}_{K}+B \hat{\sigma}_{K}^{x}\right)_{\tilde{\boldsymbol{n}}, \boldsymbol{n}}\right.\right. \\
& \left.\left.-\left(A \hat{\mathbb{1}}_{K}+B \hat{\sigma}_{K}^{x}\right)_{\boldsymbol{n}, \tilde{\boldsymbol{n}}} \hat{g}(\tilde{\boldsymbol{n}})\left(A \hat{\mathbb{1}}_{K}+B \hat{\sigma}_{K}^{x}\right)_{\tilde{\boldsymbol{n}}, \boldsymbol{n}} \circ \hat{g}(\boldsymbol{n})\right]\right\rangle_{\tilde{\boldsymbol{n}}}
\end{aligned}
$$

where the open dot indicates the time convolution [cf. Eq. (5.9)] and the time argument of the functions $A$ and $B$ is the first (second) time argument of the Green's function on their right (left), e.g.:

$$
B g B \circ g \equiv \int d t_{3} B\left(t_{1}\right) g^{K}\left(t_{1}, t_{3}\right) B\left(t_{3}\right) g^{K}\left(t_{3}, t_{2}\right)
$$

Substituting the matrix Green's function of the form (5.23), we find that the collision integral becomes:

$$
\left\langle\left(\begin{array}{l}
\widehat{\mathrm{St}}^{R} \widehat{\mathrm{St}}^{K} \\
\widehat{\mathrm{St}}^{Z} \widehat{\mathrm{St}}^{A}
\end{array}\right)\right\rangle_{\tilde{\boldsymbol{n}}}
$$

The explicit expressions for the retarded, advanced and ' $\mathrm{Z}$ ' components are:

$$
\begin{aligned}
& \widehat{\mathrm{St}}^{R}=g^{K}(\boldsymbol{n})[B ; A]+g^{K}(\boldsymbol{n}) \circ B(\boldsymbol{n}, \tilde{\boldsymbol{n}}) g^{K}(\tilde{\boldsymbol{n}}) B(\tilde{\boldsymbol{n}}, \boldsymbol{n}) \\
& \widehat{\mathrm{St}}^{A}=[A ; B] g^{K}(\boldsymbol{n})-B(\boldsymbol{n}, \tilde{\boldsymbol{n}}) g^{K}(\tilde{\boldsymbol{n}}) B(\tilde{\boldsymbol{n}}, \boldsymbol{n}) \circ g^{K}(\boldsymbol{n}) \\
& \widehat{\mathrm{St}}^{Z}=2 \delta\left(t_{1}-t_{2}\right)[A ; B]-2 B(\boldsymbol{n}, \tilde{\boldsymbol{n}}) g^{K}(\tilde{\boldsymbol{n}}) B(\tilde{\boldsymbol{n}}, \boldsymbol{n})
\end{aligned}
$$

where the (equal time) commutator is

$$
[A ; B] \equiv A(\boldsymbol{n}, \tilde{\boldsymbol{n}}) B(\tilde{\boldsymbol{n}}, \boldsymbol{n})-B(\boldsymbol{n}, \tilde{\boldsymbol{n}}) A(\tilde{\boldsymbol{n}}, \boldsymbol{n})
$$

The calculations performed so far are exact. However at one loop we are interested in terms up to the second order in the fluctuating fields. Then the expansion of the exponentials in Eq. (D1) shows that any product of two functions $B$ is proportional to terms of the form $K_{-} K_{-}$, which vanish after averaging over the fluctuating fields; accordingly, we drop such terms. The remaining terms are all commutators, whose explicit (approximate) form is:

$$
[A ; B]=2 i\left(K_{-}(\tilde{\boldsymbol{n}})-K_{-}(\boldsymbol{n})\right)
$$

Note that the second order terms cancel each other exactly. The surviving first order terms lead to Eq. (5.24) for $K_{-}$.

For reference, we present the expression for $\widehat{\mathrm{St}}^{K}$ from which Eq. (5.73) and Eq. (5.76) are derived [with the exception of the last line of Eq. (5.76b), which is a consequence of the requirement $(5.34)$ for $\left.K_{+}\right]$:

$$
\begin{aligned}
\widehat{\mathrm{St}}{ }^{K}= & 2 \delta\left(t_{1}-t_{2}\right)[A ; B]+2 A(\boldsymbol{n}, \tilde{\boldsymbol{n}}) g^{K}(\tilde{\boldsymbol{n}}) A(\tilde{\boldsymbol{n}}, \boldsymbol{n}) \\
& -g^{K}(\boldsymbol{n}) A(\boldsymbol{n}, \tilde{\boldsymbol{n}}) A(\tilde{\boldsymbol{n}}, \boldsymbol{n})-A(\boldsymbol{n}, \tilde{\boldsymbol{n}}) A(\tilde{\boldsymbol{n}}, \boldsymbol{n}) g^{K}(\boldsymbol{n}) \\
& +g^{K}(\boldsymbol{n}) B(\boldsymbol{n}, \tilde{\boldsymbol{n}}) B(\tilde{\boldsymbol{n}}, \boldsymbol{n})+B(\boldsymbol{n}, \tilde{\boldsymbol{n}}) B(\tilde{\boldsymbol{n}}, \boldsymbol{n}) g^{K}(\boldsymbol{n}) \\
& +g^{K}(\boldsymbol{n}) \circ B(\boldsymbol{n}, \tilde{\boldsymbol{n}}) g^{K}(\tilde{\boldsymbol{n}}) A(\tilde{\boldsymbol{n}}, \boldsymbol{n}) \\
& -A(\boldsymbol{n}, \tilde{\boldsymbol{n}}) g^{K}(\tilde{\boldsymbol{n}}) B(\tilde{\boldsymbol{n}}, \boldsymbol{n}) \circ g^{K}(\boldsymbol{n})
\end{aligned}
$$

\section{APPENDIX E: DERIVATION OF EQS. (5.81b) AND (5.82b)}

We start the derivation by separating the Keldysh and retarded/advanced propagators contributions to $\widehat{\mathrm{St}}_{1}{ }^{\mathrm{in}}$ in 
Eq. (5.75b):

$$
\begin{aligned}
\widehat{\mathrm{St}}_{1}^{K}= & -\frac{i}{16} \int \frac{d \boldsymbol{n}_{2} d \boldsymbol{n}_{3}}{\left(\Omega_{d}\right)^{2}} \gamma_{12}^{3} g\left(\bar{t}, \delta t, \boldsymbol{n}_{2}\right) \\
& {\left[2 \mathcal{K}^{K}\left(\bar{t}, \delta t, \boldsymbol{n}_{3}, \boldsymbol{n}_{2}\right)-\mathcal{K}^{K}\left(\bar{t}+\delta t / 2,0, \boldsymbol{n}_{3}, \boldsymbol{n}_{2}\right)\right.} \\
& \left.-\mathcal{K}^{K}\left(\bar{t}-\delta t / 2,0, \boldsymbol{n}_{3}, \boldsymbol{n}_{2}\right)-\left(\boldsymbol{n}_{2} \rightarrow \boldsymbol{n}_{1}\right)\right] \\
\widehat{\mathrm{St}}_{1}^{R A}= & -\frac{i}{32} \int d t_{3} \int \frac{d \boldsymbol{n}_{2} d \boldsymbol{n}_{3}}{\left(\Omega_{d}\right)^{2}} \gamma_{12}^{3} \\
& {\left[\mathcal{K}^{A}\left(t_{3}, t_{2}, \boldsymbol{n}_{3}, \boldsymbol{n}_{2}\right)-\mathcal{K}^{R}\left(t_{1}, t_{3}, \boldsymbol{n}_{2}, \boldsymbol{n}_{3}\right)-\left(\boldsymbol{n}_{2} \rightarrow \boldsymbol{n}_{1}\right)\right] } \\
\times & {\left[g\left(t_{1}, t_{3}, \boldsymbol{n}_{1}\right) g\left(t_{3}, t_{2}, \boldsymbol{n}_{2}\right)+g\left(t_{1}, t_{3}, \boldsymbol{n}_{2}\right) g\left(t_{3}, t_{2}, \boldsymbol{n}_{1}\right)\right] }
\end{aligned}
$$

For our convenience, we rewrote the Keldysh part in terms of the new time variables $\bar{t}, \delta t$ :

$$
\begin{aligned}
& \bar{t}=\frac{t_{1}+t_{2}}{2}, \quad \delta t=t_{1}-t_{2}, \\
& g\left(t_{1}, t_{2}\right) \rightarrow g(\bar{t}, \delta t), \mathcal{K}^{K}\left(t_{1}, t_{1}\right) \rightarrow \mathcal{K}^{K}(\bar{t}+\delta t / 2,0), \ldots
\end{aligned}
$$

Let us consider the limit $t_{2} \rightarrow t_{1}$ of Eq. (E1); clearly when $\delta t \rightarrow 0$, the square bracket vanishes. However we know that in this limit $g \rightarrow-2 i / \pi \delta t$ [cf. Eq. (5.31)]: in principle there could be a non-vanishing contribution from the first order expansion of the propagators in $\delta t$. The last two Keldysh propagators depend on $\delta t$ in their first variable, but with opposite signs, and so their respective first order terms cancel each other. As for the first propagator, the property $\mathcal{K}^{K}(1,2)=\mathcal{K}^{K}(2,1)$ translates into $\mathcal{K}^{K}(t, \delta t)=\mathcal{K}^{K}(t,-\delta t)$, which ensures the absence of first order terms. We conclude that in the limit $\delta t \rightarrow 0$ the Keldysh propagator terms vanish. Similarly from the property $\mathcal{K}^{A}(1,2)=\mathcal{K}^{R}(2,1)$ it follows that Eq. (E2) vanish for $t_{2}=t_{1}$; this concludes the proof of Eq. (5.81b).

Let us turn to Eq. (5.82b). Since $\partial_{t_{1}}-\partial_{t_{2}}=2 \partial_{\delta t}$, we must expand the Keldysh propagators to the second order in $\delta t$. At this order the square bracket in Eq. (E1) is (up to the proper combination of angular variables):

$$
\delta t^{2}\left(\partial_{\delta t}^{2}-\frac{1}{4} \partial_{\bar{t}}^{2}\right) \mathcal{K}^{K}(\bar{t}, 0)=-\delta t^{2} \lim _{t_{2} \rightarrow t_{1}} \partial_{t_{1}} \partial_{t_{2}} \mathcal{K}^{K}\left(t_{1}, t_{2}\right)
$$

where in the last equality we restored the original time variables. In the operator notation this is:

$$
\delta t^{2}\left[\partial_{t} \mathcal{K}^{K} \partial_{t}\right]_{t_{2}=t_{1}}
$$

Therefore

$$
\begin{aligned}
& \lim _{t_{2} \rightarrow t_{1}}\left(\partial_{t_{1}}-\partial_{t_{2}}\right){\widehat{\mathrm{St}_{1}}}_{1}^{K}\left(t_{1}, t_{2}\right)=\frac{1}{4 \pi} \int \frac{d \boldsymbol{n}_{2} d \boldsymbol{n}_{3}}{\Omega_{d}^{2}} \gamma_{12}^{3} \\
& \quad\left\{\left[\partial_{t} \mathcal{K}^{K} \partial_{t}\right]\left(t_{1}, t_{1}, \boldsymbol{n}_{3}, \boldsymbol{n}_{1}\right)-\left[\partial_{t} \mathcal{K}^{K} \partial_{t}\right]\left(t_{1}, t_{1}, \boldsymbol{n}_{3}, \boldsymbol{n}_{2}\right)\right\}
\end{aligned}
$$

which proves the first part of Eq. (5.82b).

As for Eq. (E2), using again the analytic property $\mathcal{K}^{A}(1,2)=\mathcal{K}^{R}(2,1)$ we conclude that when the derivatives $\partial_{t_{1}}, \partial_{t_{2}}$ act on the distribution functions $g$, the terms in the second line cancel each other. However there are non-vanishing contributions when a derivative acts on the propagators, such as:

$$
\begin{aligned}
& \int d t_{3} \int \frac{d \boldsymbol{n}_{2} d \boldsymbol{n}_{3}}{\Omega_{d}^{2}} \gamma_{12}^{3} g\left(t_{1}, t_{3}, \boldsymbol{n}_{1}\right) g\left(t_{3}, t_{1}, \boldsymbol{n}_{2}\right) \\
& \times\left\{\left[\partial_{t} \mathcal{K}^{R}\right]\left(t_{1}, t_{3}, \boldsymbol{n}_{1}, \boldsymbol{n}_{3}\right)-\left[\partial_{t} \mathcal{K}^{R}\right]\left(t_{1}, t_{3}, \boldsymbol{n}_{2}, \boldsymbol{n}_{3}\right)\right\}
\end{aligned}
$$

Collecting all the terms we arrive at:

$$
\begin{aligned}
& \lim _{t_{2} \rightarrow t_{1}}\left(\partial_{t_{1}}-\partial_{t_{2}}\right) \widehat{\mathrm{St}}_{1}^{R A}\left(t_{1}, t_{2}\right)=\frac{i}{16} \int d t_{3} \int \frac{d \boldsymbol{n}_{3} d \boldsymbol{n}_{2}}{\Omega_{d}^{2}} \gamma_{12}^{3} \\
& \left\{\left[\partial_{t} \mathcal{K}^{R}\right]\left(t_{1}, t_{3}, \boldsymbol{n}_{2}, \boldsymbol{n}_{3}\right)-\left[\partial_{t} \mathcal{K}^{R}\right]\left(t_{1}, t_{3}, \boldsymbol{n}_{1}, \boldsymbol{n}_{3}\right)\right\} \\
& \times\left[g\left(t_{1}, t_{3}, \boldsymbol{n}_{1}\right) g\left(t_{3}, t_{1}, \boldsymbol{n}_{2}\right)+g\left(t_{1}, t_{3}, \boldsymbol{n}_{2}\right) g\left(t_{3}, t_{1}, \boldsymbol{n}_{1}\right)\right]
\end{aligned}
$$

which concludes the derivation of Eq. (5.82b).

\section{APPENDIX F: ELASTIC KERNELS IN TERMS OF THE INTERACTION PROPAGATOR $D$}

To compare the kernels in Eq. (6.9) to the corresponding expressions in Ref. 17, we use the Fourier transforms of Eqs. (5.51) and (5.55a) to obtain

$$
\operatorname{Re}\left[\hat{\mathcal{L}}^{\rho}-\hat{\mathcal{L}}^{g}\right]=-\nu \omega \operatorname{Im}\left[\hat{\mathcal{L}}^{g} \hat{D}^{R} \hat{\mathcal{L}}^{g}\right]
$$

If we assume, as is done in Ref. 17, that the Fermi liquid parameters do not depend on the momentum direction, then the interaction propagators $D^{R, A}$ also do not depend on it and the above equation becomes

$$
\operatorname{Re}\left[\mathcal{L}^{\rho}-\mathcal{L}^{g}\right]=-\nu \omega \operatorname{Im}\left[\mathcal{L}^{g}\right\rangle D^{R}\left\langle\mathcal{L}^{g}\right]
$$

where we generalized the angular integral notation so that

$$
\left\langle\mathcal{L}^{g}=\int \frac{d \boldsymbol{n}_{1}}{\Omega_{d}} \mathcal{L}^{g}\left(\boldsymbol{n}_{1}, \boldsymbol{n}_{2}\right), \quad \mathcal{L}^{g}\right\rangle=\int \frac{d \boldsymbol{n}_{2}}{\Omega_{d}} \mathcal{L}^{g}\left(\boldsymbol{n}_{1}, \boldsymbol{n}_{2}\right)
$$

We remind that our ghost propagator $\mathcal{L}^{g}$ coincides with the diffuson propagator $D$ of Ref. 17 .

By substituting Eq. (F2), we rewrite the kernels (6.9) as:

$$
\begin{aligned}
& \mathcal{S}_{\mu \nu}^{11}(\omega)=\frac{2}{\pi} \delta_{\mu \nu} \int \frac{d^{d} q}{(2 \pi)^{d}}\left(\left\langle\mathcal{L}^{g}\right\rangle\left\langle\mathcal{L}^{g}\right\rangle-\left\langle\mathcal{L}^{g} \mathcal{L}^{g}\right\rangle\right) D^{R} \\
& \mathcal{S}_{\mu \nu}^{12}(\omega)=-\frac{2 d}{\pi} \int \frac{d^{d} q}{(2 \pi)^{d}}\left\langle n_{\alpha} \mathcal{L}^{g}\right\rangle\left\langle\mathcal{L}^{g} n_{\beta}\right\rangle D^{R} \\
& \mathcal{E}_{\mu \nu}(\omega)=\frac{d}{\pi \tau} \operatorname{Im} \int \frac{d^{d} q}{(2 \pi)^{d}} D^{R}\left[\left\langle\mathcal{L}^{g} n_{\alpha} \mathcal{L}^{g} n_{\beta}\right\rangle\left\langle\mathcal{L}^{g}\right\rangle\right. \\
& -\left\langle\mathcal{L}^{g} n_{\alpha} \mathcal{L}^{g}\right\rangle\left\langle n_{\beta} \mathcal{L}^{g}\right\rangle+\left\langle\mathcal{L}^{g} n_{\alpha}\right\rangle\left\langle\mathcal{L}^{g} n_{\beta}\right\rangle\left\langle\mathcal{L}^{g}\right\rangle \\
& \left.-\left\langle\mathcal{L}^{g}\right\rangle\left\langle n_{\alpha} \mathcal{L}^{g} n_{\beta}\right\rangle\left\langle\mathcal{L}^{g}\right\rangle\right]+D^{R}\left[\left\langle\mathcal{L}^{g}\right\rangle\left\langle n_{\alpha} \mathcal{L}^{g} n_{\beta} \mathcal{L}^{g}\right\rangle\right. \\
& \left.-\left\langle\mathcal{L}^{g} n_{\alpha}\right\rangle\left\langle\mathcal{L}^{g} n_{\beta} \mathcal{L}^{g}\right\rangle-\left\langle\mathcal{L}^{g} n_{\alpha} \mathcal{L}^{g} n_{\beta} \mathcal{L}^{g}\right\rangle\right]
\end{aligned}
$$


The first square bracket in the kernel $\mathcal{E}$ can be expressed as

$$
\tau\left[\left\langle\mathcal{L}^{g} n_{\alpha} \mathcal{L}^{g}\left[n_{\beta} ; \widehat{\mathrm{St}}_{\tau}\right] \mathcal{L}^{g}\right\rangle+\left\langle\mathcal{L}^{g}\left[n_{\alpha} ; \widehat{\mathrm{St}}_{\tau}\right] \mathcal{L}^{g} n_{\beta}\right\rangle\left\langle\mathcal{L}^{g}\right\rangle\right]
$$

and using the identity:

$$
\mathcal{L}^{g}\left[\boldsymbol{n} ; \widehat{\mathrm{St}}_{\tau}\right] \mathcal{L}^{g}=\left[\boldsymbol{n} ; \mathcal{L}^{g}\right]
$$

we recast it as follows:

$$
\begin{aligned}
& \tau\left[\frac{\delta_{\mu \nu}}{d}\left(\left\langle\mathcal{L}^{g} \mathcal{L}^{g}\right\rangle-\left\langle\mathcal{L}^{g}\right\rangle\left\langle\mathcal{L}^{g}\right\rangle\right)\right. \\
& \left.\quad+\left\langle\mathcal{L}^{g}\right\rangle\left\langle n_{\alpha} \mathcal{L}^{g} n_{\beta}\right\rangle-\left\langle\mathcal{L}^{g} n_{\alpha} \mathcal{L}^{g} n_{\beta}\right\rangle\right]
\end{aligned}
$$

In the second square bracket we use the identity

$$
\mathcal{L}^{g} \boldsymbol{n} \mathcal{L}^{g}=\frac{i}{v_{F}} \partial_{\boldsymbol{q}} \mathcal{L}^{g}
$$

to obtain:

$$
\frac{i}{v_{F}}\left[\left\langle\mathcal{L}^{g}\right\rangle \partial_{q_{\beta}}\left\langle n_{\alpha} \mathcal{L}^{g}\right\rangle-\left\langle\mathcal{L}^{g} n_{\alpha}\right\rangle \partial_{q_{\beta}}\left\langle\mathcal{L}^{g}\right\rangle-\left\langle\mathcal{L}^{g} n_{\alpha} \partial_{q_{\beta}} \mathcal{L}^{g}\right\rangle\right]
$$

Finally the identity

$$
\mathcal{L}^{g} \mathcal{L}^{g}=-i \partial_{\omega} \mathcal{L}^{g}
$$

enables us to conclude that the sum of the three kernels

$$
\mathcal{S}^{11}+\mathcal{S}^{12}+\mathcal{E}
$$

that determines the correction to the conductivity Eq. (6.8) - coincides with the combination $\left(K_{0}-K_{1}-\right.$ $\left.L_{0} / v_{F} \tau\right)$ in the expression for the conductivity of Ref. 17.

\section{APPENDIX G: THE INELASTIC KERNEL FOR THE PHASE RELAXATION TIME}

Let us consider a uniform system in which the bosons are assumed to be in equilibrium with the electrons. In other words, the distribution function $f$ is independent of $\boldsymbol{r}, \boldsymbol{n}$ and the boson-electron collision integral (3.18) must vanish. The latter condition enables us to express the bosonic distributions $N^{\alpha}$ in terms of $f$ and to obtain

$$
\Upsilon_{i j}(\epsilon, \omega)=-\Omega_{d} \delta\left(\widehat{\boldsymbol{n}_{i} \boldsymbol{n}_{j}}\right) \frac{1}{\omega} \int d \epsilon_{1} \Psi\left(\epsilon, \epsilon_{1} ; \omega\right)
$$

[from now on irrelevant angular and momentum variables are omitted; all relevant definitions can be found in Sec. III]. The former condition implies that the collision integrals $(3.19 \mathrm{~d}),(3.20 \mathrm{~b})$ and (3.20f) vanish and therefore the kinetic equation for (the zeroth harmonic of) $f$ reduces to

where

$$
\partial_{t} f(\epsilon ; t)=\int d \omega \int d \epsilon_{1} A(\omega) \Psi\left(\epsilon, \epsilon_{1} ; \omega\right)
$$

$A(\omega)=\frac{-2}{\nu \pi \omega^{2}} \operatorname{Re} \operatorname{Tr}\left\{\left[2 \widehat{\mathrm{St}}_{\tau} \hat{\overline{\mathcal{L}}}^{g}+\hat{1}\right] \widehat{\mathrm{St}}_{\tau}\left[\hat{\mathcal{L}}^{\rho}+3 \hat{\mathcal{L}}^{\sigma}-4 \hat{\mathcal{L}}^{g}\right]\right\}$

We substitute Eq. (F1) and the similar relation for the triplet channel $\left[D_{T}^{R}\right.$ being the triplet channel propagator] into the expression for $A(\omega)$; then we use the identity (5.44) and obtain

$$
A(\omega)=-\frac{2}{\pi \omega} \operatorname{Im} \operatorname{Tr}\left[\widehat{\mathrm{St}}_{\tau} \hat{\overline{\mathcal{L}}}^{g}\left(\hat{D}^{R}+\hat{D}_{T}^{R}\right) \hat{\mathcal{L}}^{g}\right]
$$

Using again Eq. (5.44), we recover immediately the form of the inelastic kernel $A(\omega)$ given in Ref. 27.
1 G. Wiedemann and R. Franz, Ann. Phys., Lpz., (2), 89, 497 (1853).

2 A.A. Abrikosov, Fundamentals of the Theory of Metals (North-Holland, New York, 1988).

3 E.M. Lifshitz and L.P. Pitaevskii, Physical kinetics (Pergamon Press, New York, 1981).

4 R. Kubo, J. Phys. Soc. Japan 12, 570 (1957).

5 J.M. Luttinger, Phys. Rev. 135, 1505 (1964).

6 K. Maki, Progr. Theor. Phys. 39, 897 (1968).

7 R.S. Thompson, Phys. Rev. B 1, 327 (1970).

8 L.G. Aslamazov and A.I. Larkin, Sov. Phys. Solid State 10, 875 (1968).

9 L.P. Gorkov, A.L. Larkin and D.E. Khmelnitskii JETP Letters 30, 228 (1979).

10 B.L. Altshuler and A.G. Aronov, Zh. Eksp. Teor. Fiz. 77, 2028 (1979) [Sov. Phys. JETP, 50, 968 (1979)].

11 B.L. Altshuler, A.G. Aronov and P.A. Lee, Phys. Rev. Lett. 44, 1288 (1980).

12 B.L. Altshuler and A.G. Aronov, in Electron-Electron In- teractions in Disordered Systems, edited by A.L. Efros and M. Pollak (North-Holland, Amsterdam, 1985).

13 I.L. Aleiner and A.I. Larkin, Phys. Rev. B 54, 14423 (1996).

14 A.M. Finkelstein, Zh. Eksp. Teor. Fiz. 84, 168 (1983) [Sov. Phys. JETP 57, 97 (1983)]; Z. Phys. B: Condens. Matter 56, 189 (1984).

15 A.M. Rudin, I.L. Aleiner and L.I. Glazman, Phys. Rev. B 55, 9322 (1997).

16 I.L. Aleiner, B.L. Altshuler and M.E. Gershenson, Waves Random Media 9, 201 (1999).

17 G. Zala, B.N. Narozhny, I.L. Aleiner, Phys. Rev. B 64, 214204 (2001).

18 I.V. Gornyi and A.D. Mirlin, Phys. Rev. B 69, 045313 (2004).

19 C. Castellani, C. DiCastro, G. Kotliar, P.A. Lee and G. Strinati, Phys. Rev. B 37, 9046 (1988).

20 D.V. Livanov, M.Yu. Reizer and A.V. Sergeev, Zh. Eksp. Teor. Fiz. 99, 1230 (1991) [Sov. Phys. JETP 72, 760 
(1991)]

21 J.S. Langer, Phys. Rev. 128, 110 (1962).

22 D.R. Nieven and R.A. Smith, cond-mat/0301451.

23 R. Raimondi, G. Savona, P. Schwab and T. Lück, cond-mat/0402245.

24 G. Eilenberger, Z. Phys. B 214, 195 (1968); A.I. Larkin and Y.N. Ovchinnikov, Zh. Eksp. Teor. Fiz. 55, 2262 (1968) [Sov. Phys. JETP 28, 1200 (1969)]; A.I. Larkin and Y.N. Ovchinnikov, Zh. Eksp. Teor. Fiz. 73, 299 (1977) [Sov. Phys. JETP 46, 155 (1977)].

25 A. Kamenev and A. Andreev, Phys. Rev. B 60, 2218 (1999).

26 A.A. Abrikosov, L.P. Gorkov and I.E. Dzyaloshinski, Methods of Quantum Field Theory in Statistical Physics (Prentice-Hall, Englewood Cliffs, NJ, 1963).

27 B.N. Narozhny, G. Zala, I.L. Aleiner, Phys. Rev. B 65, 180202(R) (2002).

28 D. Coffey and K.S. Bedell, Phys. Rev. Lett. 71, 1043 (1993); G.Y. Chitov and A.J. Millis, Phys. Rev. B 64, 054414 (2001).

29 A.O. Lyakhov and E.G. Mishchenko, Phys. Rev. B 67, 041304 (2003).

30 J.W.P. Hsu, A. Kapitulnik and M.Yu. Reizer, Phys. Rev. B 40, 7513 (1989).

31 M.Yu. Reizer and A. Sergeev, Phys. Rev. B 50, 9344 (1994).

32 A. Vilenkin and P.L. Taylor, Phys. Rev. B 18, 5280 (1971); M. Reizer, A. Sergeev, J.W. Wilkins and D.V. Livanov, Annals of Physics 257, 44 (1997).

33 See e.g. Refs. 12, 26 and references therein.

${ }^{34}$ The derivation similar to that of this appendix was performed independetly by M.Yu. Reyzer (private communication).

${ }^{35}$ It was shown by G.V. Chester and A. Thellung, that Eq. (1.1) remains valid for arbitrary scattering strength as long as the scattering rates and the density of states are smooth $\left(\mathcal{C}_{2}\right)$ function of energy near the Fermi level.

36 The quantum kinetic equation with the necessary conservation laws was not actually derived in Ref. 20, so we will not be able to compare their approach with ours.
37 As given in Eq. (3.1), the equilibrium distribution functions $N_{e q}^{\alpha}(\omega)$ are defined only for $\omega>0$; for $\omega<0$ they are found by using the property in Eq. (3.2).

38 The current operator used in Ref. 21 does not satisfy the continuity equation for the long range interaction potential.

39 These solution are exact only in the absence of the electric field, since in its gauge invariant form the operator acting on $\delta g_{ \pm}$is the same that appears on the left hand side of Eq. (5.71). We could include perturbatively field dependent corrections to our expressions, which would be of the first order in $\boldsymbol{E}$ for $\delta g_{-}$and of the second order for $\delta g_{+}$[since $\delta g_{+}$vanishes in equilibrium]. However, as noted before, the first property in Eq. (5.79) implies that these corrections cannot contribute to the physical quantities we are interested in and therefore we do not include them in our calculations.

40 The operator $\hat{M}$ is gauge-invariant and it has the same form in terms of $g$ or $g^{K}$.

41 by the simple substitution $\mathcal{L}^{\rho}-\mathcal{L}^{g} \rightarrow \mathcal{L}^{\rho}+3 \mathcal{L}^{\sigma}-4 \mathcal{L}^{g}$ in the kernels.

42 The Boltzmann equation description of strictly onedimensional systems is not applicable and considering the quasi one-dimensional ballistic case within our scheme is meaningless, because of the effects of boundary scattering. The ballistic regime in three dimensions also can not be considered within our scheme, because the main effect on the thermal conductivity is due to the inelastic scattering processes with momentum transfer of the order of $k_{F}$.

43 We choose to collect the bosonic contributions into a single kernel so that the resulting momentum integral is convergent.

44 They can be obtained from Eqs. (6.25)-(6.26) in the limit $d \rightarrow 2$.

45 A similar argument is presented in Ref. 21.

46 This definition of the density of states is one half of the one given in Eqs. (2.19), because of the different limits for the $\omega$ integration in the energy density and the specific heat. 